\title{
Surgical Management of Complex Spinal Cord Lipomas : A New Perspective
}

\author{
Dachling Pang ${ }^{1,2}$ \\ Department of Paediatric Neurosurgery, Great Ormond Street Hospital for Children, NHS Trust, London, UK \\ Department of Paediatric Neurosurgery, ${ }^{2}$ University of California, Davis, CA, USA
}

This review summarises the classification, anatomy and embryogenesis of complex spinal cord lipomas, and describes in some detail the new technique of total lipoma resection and radical reconstruction of the affected neural placode. Its specific mission is to tackle two main issues surrounding the management of complex dysraphic lipomas : whether total resection confers better long term benefits than partial resection, and whether total resection does better than conservative treatment, i.e., no surgery, for asymptomatic lipomas. Accordingly, the 24 years progression-free survival data of the author and colleagues' series of over 350 cases of total resection are compared with historical data from multiple series (including our own) of partial resection, and total resection data specifically for asymptomatic lesions are compared with the two known series of non-surgical treatment of equivalent patients. These comparisons amply support the author's recommendation of total resection for most complex lipomas, with or without symptoms. The notable exception is the asymptomatic chaotic lipoma, whose peculiar anatomical relationship with the neural tissue defies even our aggressive surgical approach, and consequently projects worse results (admittedly of small number of cases) than for the other two lipoma subtypes of dorsal and transitional lesions. Prophylactic resection of asymptomatic chaotic lipomas is therefore not currently endorsed.

Key Words : Progression-free survival · Spinal dysraphism.

\section{INTRODUCTION}

Two issues concerning lipomas continue to provoke debates among paediatric neurosurgeons : one is whether asymptomatic lipomas should be treated with prophylactical surgery; the other is with what surgery.

Regarding the first issue, arguments continue unabated between the "cutters" (those who advocate early resection) and the "non-cutters" (those who would do surgery only after symptoms appear), but one thing is beyond dispute : spinal cord lipoma is a progressive disease. In 2004, Kulkarni et al. ${ }^{27)}$, from L'Hopital Necker-Enfants Malades, Paris, published a prospective study of a relatively large cohort of children with lipomas that were followed without surgery and concluded that asymptomatic lipomas have a 33\% chance of deterioration over 9 years. A comparable though retrospective study from London in $2012^{73)}$ similarly reported a $40 \% 10$ year deterioration rate in unoperated asymptomatic lipomas. The Lon-

- Received : January 28, 2020 •Revised : April 28, 2020 •Accepted : April 28, 2020

- Address for reprints : Dachling Pang

Department of Paediatric Neurosurgery, Great Ormond Street Hospital for Children, NHS Trust, Great Ormond Street, London, WC1N3JH, UK Tel : +44-20 7405 9200, Fax : +44-20 7813 8279, E-mail : pangtv@aol.com, ORCID : https://orcid.org/0000-0002-6603-6546

This is an Open Access article distributed under the terms of the Creative Commons Attribution Non-Commercial License (http://creativecommons.org/licenses/by-nc/4.0) which permits unrestricted non-commercial use, distribution, and reproduction in any medium, provided the original work is properly cited. 
don study also showed that females with transitional lipomas and/or a terminal syrinx fared even worse. Thus, since half of their cohort were females and approximately $70 \%$ of their lipomas were of the transitional type, the poor outcome rate of the group, when projected to a larger cohort size and longer follow-up, may well exceed $40 \%$. Such dire statistics for untreated lipomas in children with presumably long actuarial survival seem to demand some type of intervention. Ironically, the Parisian group advised against prophylactic surgery because their own results of prophylactic partial resection in a later report showed an even higher probability of late deterioration than the group with no surgery ${ }^{27,63)}$. The logical conclusion, considering the high progression rates of both the natural disease and partial resection, is that asymptomatic lipomas do indeed need surgery, just not partial resection.

Which brings in the second issue of what might be the most effective surgery that offers long term benefits over the natural history of complex lipomas. Historically, the traditional technique had always been partial resection ${ }^{1,6,19,22,29,40,70)}$, but despite its wide usage, data from most large series do not show that it offers adequate long-term protection against disease progression. For example, Dorward et al. ${ }^{16)}$ reported a symptomatic recurrence rate of $48 \%$ over merely 2.2 years. Colak et al. ${ }^{11)}$ reported a $52 \%$ recurrence rate over 10 years with partial resection, but their series comprised 37\% terminal lipomas, which are known to have much better prognosis than dorsal and transitional lipomas, implying that the progression rate if calculated just for the latter lipoma types must be even higher than $52 \%$. Pierre-Kahn et al. ${ }^{62)}$ documented a 10 years recurrence of $46 \%$ but again their series contains many terminal and filar lipomas; and Cochrane et al. ${ }^{10)}$ and Xenos et al. ${ }^{74)}$ similarly recorded early recurrence. These disturbing results pose the obvious question whether leaving behind a large amount of residual fat with its broad, raw, and sticky abraded surface actually provokes new adhesions to the adjacent dura and consequently incurs earlier and firmer retethering than if the lipoma had been left untouched. Or alternatively, whether complete removal of the bulky lipoma plus measures to render the now pliant neural placode less "sticky", would impart greater permanent benefits than partial resection.

The need for a different procedure other than partial resection occurred to me in 1991, when my own series of 116 partial resections following conventional teaching, ${ }^{4,19,40,41,62,67,70)}$ (we were taught always to leave a goodly layer of fat on the neural placode and that exposing even a glimmer of cord tissue through the fat was a sin) showed an alarming 65\% symptomatic recurrence rate over 11 years. This untenable situation compelled me to entertain the then iconoclastic concept of complete resection of the lipoma, strategic reconstruction of the neural placode, and expansile duraplasty. My rationale for this more aggressive approach ${ }^{59)}$ is founded on three hypotheses : 1) the high recurrence rate after partial resection is due to re-tethering at the resection site; 2 ) re-tethering is in turn promoted by three conditions : a tight content-container relationship between the affected placode and its adjacent intradural space, a broad "sticky" slab of remaining fat, and incomplete separation of the terminal placode from the caudal fat (see below); and 3) total resection eliminates these conditions contributory to re-tethering, and thus reduces the probability of late deterioration. The object of this proposed new operation is therefore to create a milieu least conducive to re-sticking of the neural placode. The first premiss towards this goal is predicated on the observation that the spinal cord normally shows considerable intradural motion to gravity and postural changes demonstrable on dynamic imaging ${ }^{5,15)}$. Lowering the content-container ratio and amplifying the amount of free placode movements within the dural sac should therefore reduce sustained contacts between placode and dura, hence also the probability of adhesion. To achieve this in a "virgin" (previously unoperated) lipoma, the bulk of the lipoma-cord composite must first be severely trimmed by resecting all or most of the engorging fat down to the thin, supple neural placode; for "redo" lesions that have had previous surgery, the stiff fibrous scar must also be removed. The goal is to render the thinnest, most malleable neural placode so that through tensionless pia-to-pia neurulation (see below), a slender but unstrangled, pia-covered neural tube can be created. The sticky lipoma bed is thus concealed within this tube, and the dural sac is then made capacious by a generous graft. Finally, total elimination of the obscuring fat will also enhance the chances of terminal untethering.

In 2009 and 2010 59,60), my colleagues and I published our early series of 238 patients who had total/near-total resection of complex lipomas and compared their short and long term outcomes with our prior series of 116 patients with partial resection. In 2013, we updated our series of total resection to 315 cases $^{60)}$, and recently, our number has risen to over 450 cas$\mathrm{es}^{53,55)}$. Our ever enlarging data pool unfailingly shows an 
overwhelming advantage of total over partial resection in almost all respects, but the subset of asymptomatic virgin lipomas continues to pique uncertainties and controversies among some paediatric neurosurgeons. This review therefore re-examines three recurring questions : 1 ) is total resection better than partial resection for both symptomatic and asymptomatic lipomas?; 2) is total resection better than no surgery for asymptomatic lipomas, i.e., should they all be treated prophylactically with total resection?; and 3) is partial resection worse than no surgery and, if so, should it be strongly discouraged?

\section{ANATOMY AND CLASSIFICATION}

There is an increasing body of literature proposing newer and newer nomenclatures and classifications of spinal cord lipomas but they are essentially variations of the same existing scheme. Here, we are sticking to our old classification, solidly based on embryogenesis, as follows.

\section{Dorsal lipoma}

A dorsal lipoma perches entirely on the dorsal surface of the

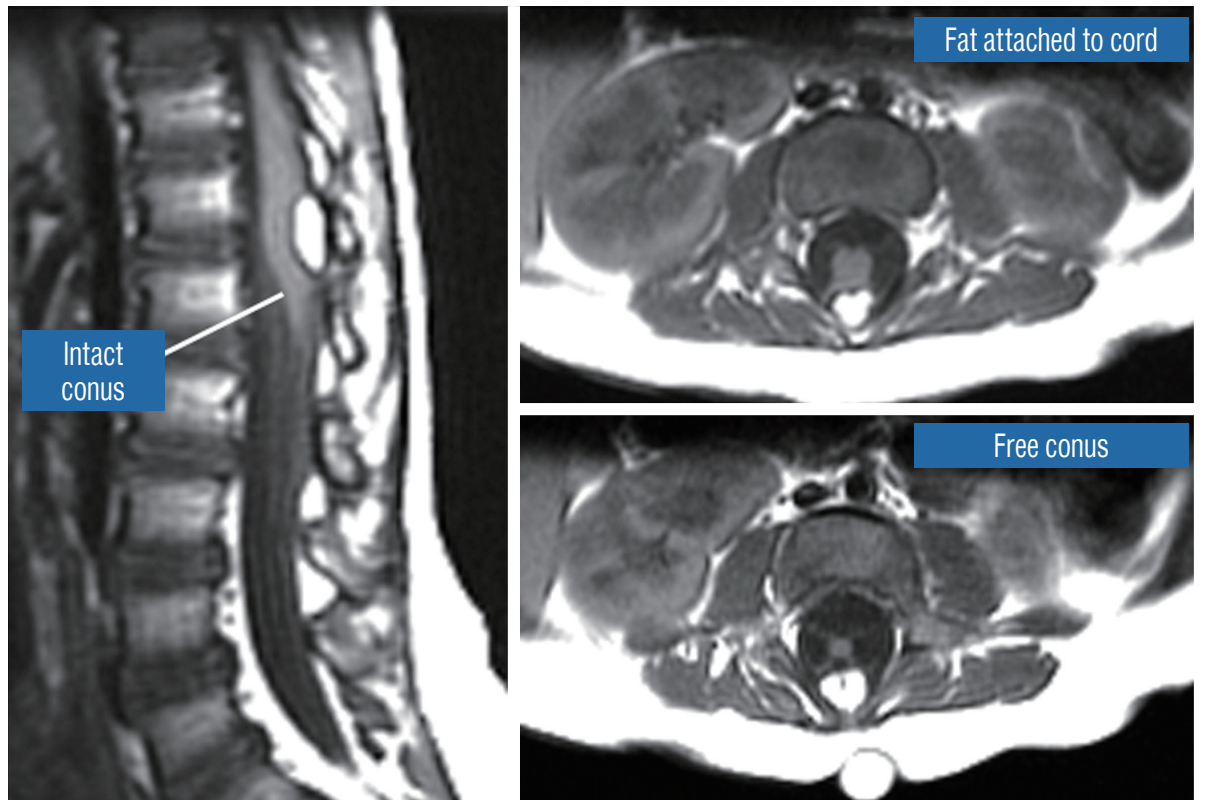

Fig. 1. Dorsal lipoma on magnetic resonance imaging. Sagittal image shows intact conus caudal to lipoma stalk. Axial images : upper shows site of lipoma attachment to cord; lower shows free conus just caudal to the level of lipoma attachment. Reprinted from Pang et al. ${ }^{61)}$ with permission from Springer Nature.
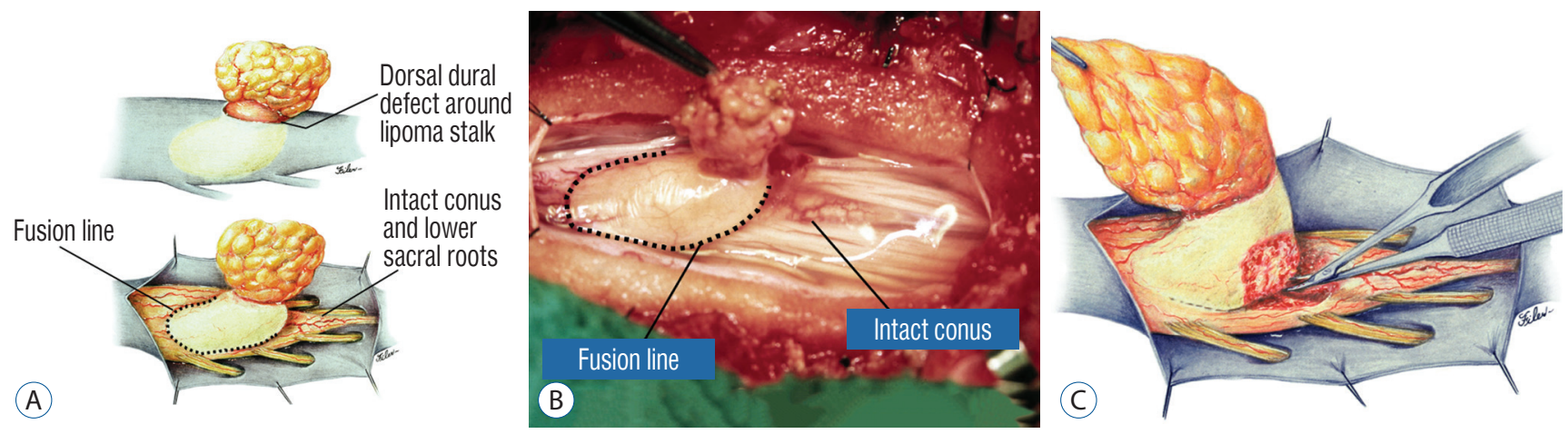

Fig. 2. Dorsal lipoma. A : Intraoperative drawings : upper shows neat dorsal dural defect through which lipoma stalk goes. Lower shows circumferential fusion line and intact conus. B : Intraoperative picture shows neat oval fusion line around lipoma-cord interface on a horizontal plane. Note intact conus and caudal sacral roots. C : Resection of dorsal lipoma can be executed with a completely circumscribed perspective from all sides of the fusion line, impossible with transitional lipoma. Reprinted from Pang et al. ${ }^{61)}$ with permission from Springer Nature. 
lumbar spinal cord, and never involves the conus (Fig. 1). The junction between lipoma, spinal cord, and pia, the fusion line (see below), is usually traceable along a roughly oval track, demarcating fat from the more lateral dorsal root entry zone (DREZ) and nerve roots (Fig. 2A and B), which means the nerve roots and DREZ are never mixed up with the lipoma. The lipomatous stalk usually traverses a fairly discrete defect in the dorsal dura to blend with the subcutaneous fat. The uninvolved conus often ends in a thickened filum terminale. An idealised depiction of the resection of a typical dorsal lipoma is shown in Fig. 2C, which illustrates a clear view of the entire circumference of the fusion line, and how the surgeon can command a full $360^{\circ}$ approach to the lipoma-cord interface.

\section{Transitional lipoma}

The rostral part of a transitional lipoma resembles a dorsal lipoma in that it features a discrete fusion line and a reasonably organized array of nerve roots and DREZ, but unlike the dorsal type, the fat plane of a transitional lipoma then cuts ventro-caudally to involve the entire conus likened to making an oblique, bevelled knife-cut across the end of a rod (Fig. 3A). Also, unlike the dorsal type, its lipoma-cord interface is often not horizontally levelled, and the neural placode may be so rotated in its longitudinal axis that it literally spins into a parasagittal orientation, but the neural tissue is always recognisable "ventral" to this interface, so that the DREZ and nerve roots are lateral and ventral to the fusion line and therefore also do not usually traverse the lipoma (Fig. 3B). An idealised depiction of total resection of a transitional lipoma thus shows a ventrally-slanting oblique plane of resection set by the fusion line (Fig. 3C). A filum may or may not be present, and the dural defect often extends way past the neural placode to involve the caudal dural sac.

In general, dorsal lipomas conform to the "standard" architecture described above, and are mostly horizontal and symmetrical. Transitional lipomas, on the other hand, tend more to be "irregular" and unpredictable : many are not symmetrical or flat, the larger ones often overhang the nerve roots and will require special handling; congenitally defunct nerve roots do sometimes run through the lipoma and must be correctly recognized and dispensed with; and monstrous transitional lipomas have been known to overwhelm any earnest attempt to sort out the "good" anatomy.

\section{Terminal lipoma}

All terminal lipomas are sharply attached across the base of the conus without ever involving the dorsal parts of the spinal cord or nerve roots. Thus the lipoma-cord interface lies entirely below all the functional sacral roots and conus, which essentially looks normal except for the attached fat at the end. The dural sac and the overlying myofascial layers are intact. The lipoma either replaces the filum entirely, or is embedded within a portion of the filum, in which case it is often called a filar lipoma.

The excision of terminal lipoma is relatively simple and its prognosis is far better than the other types; both are beyond the scope of this review.
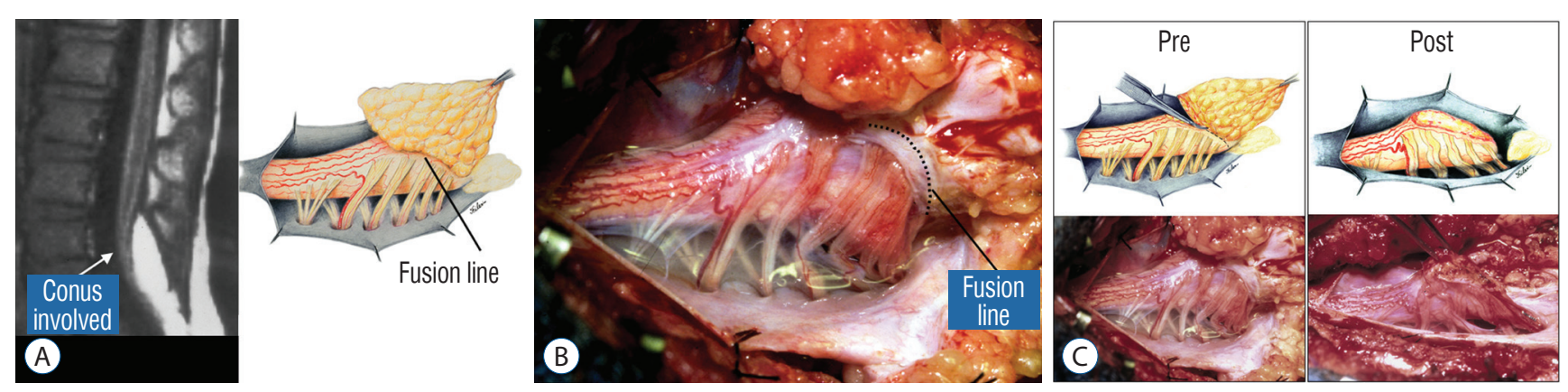

Fig. 3. Transitional lipoma. A : Left : sagittal MRI shows lipoma begins dorsally but involves entire conus. Ventral side of neural placode is free of fat. Right : the plane of the fusion line begins dorsally then cuts obliquely towards the tip of the conus. The array of DREZ and dorsal roots is also forced to slant dorso-ventrally. $\mathrm{B}$ : Intraoperative picture showing massive lipoma but very distinct dorso-ventral fusion line separating fat from the DREZ and dorsal roots, which always lie lateral and ventral to the fusion line. The ventral side of the placode is always free of fat in a regular transitional lipoma. C: Top : idealised drawings of pre- and post-resection of a relatively "standard" transitional lipoma, along an asymmetrical and oblique plane bound by the fusion line on each side, over an occasionally undulating lipoma-cord interface. Bottom shows pre- and post-resection intraoperative pictures. Reprinted from Pang et al. ${ }^{61)}$ with permission from Springer Nature. MRI : magnetic resonance imaging, DREZ: dorsal root entry zone. 


\section{Chaotic lipoma}

This novel type the author and colleagues introduced in $2009^{59)}$ is so named because neither its embryogenesis nor its anatomy "follows the rules" of the other lipoma types. Its rostral portion may look disarmingly orthodox like the dorsal or transitional lipoma, but the fat of its caudal portion percolates through the neural placode to its ventral side and always engulfs neural tissue and nerve roots to some degree (Fig. 4A). The fusion line may be visible in its rostrodorsal portion but becomes undefinable caudally as it vanishes behind the ventral fat, where the DREZ and nerve roots are no longer easily identifiable. The moniker "chaotic" depicts the confusing blend of ventral fat and neural placode, and the often impossible task of differentiating the wandering fat from the invisible neural tissue and nerve roots at surgery (Fig. 4B). Chaotic lipomas are uncommon but are typically associated with sacral agenesis $^{59)}$.

The literature ${ }^{1,62)}$ mentions one other lipoma type, the lipomyelomeningocoele, in which the caudal spinal cord and parts of the lipoma extrude dorsally out of the spinal canal, together with a an outpouching of the cerebrospinal fluid (CSF) sac (Fig. 5). The basic configuration of the lipoma is not different from that of a conventional transitional or dorsal lesion. Accordingly, we choose to include this variant subtype as either a transitional or dorsal lipoma with a descriptive qualifier of "extraspinal extension".

\section{SURGICALLY RELEVANT EMBRYOLOGY}

An understanding of the embryogenesis of lipomas is helpful in appreciating the surgical nuances.

\section{Embryogenesis of dorsal and transitional lipomas}

In the human embryo, a gradually increasing longitudinal disparity develops between the spinal cord and vertebral column because of their unequal growth rates ${ }^{14,17,28,69)}$. Allowing for some shortening of the caudal neural tube by programmed apoptosis during secondary neurulation, the tip of the cord is still noted to have ascended a fair distance from opposite the coccyx in the $30 \mathrm{~mm}$ embryo to approximately the $\mathrm{L}_{2}$ level at birth $^{3,23,28,69)}$. Smooth upward movement requires a well-constructed neural tube within a smooth dural tunnel. If during the ascent a dorsal defect develops in the dura and neural tube, the surrounding mesenchyme will invade through the defect and form a fibrofatty stalk that attaches to the sliding neural tube to cause its entrapment. This embryogenetic sequence implies a focal failure in neural tube closure during primary neurulation (secondary neurulation does not involve neural folds fusion), and thus applies only to the dorsal and transitional lipomas (see below). It also explains why these two lipoma types are always associated with adjacent bifid neural arches.

Before completion of primary neurulation, the cutaneous and neural ectoderms must first separate from each other, but this process, called disjunction, does not occur until after the midline fusion of the dorsal neural folds. This time sequence

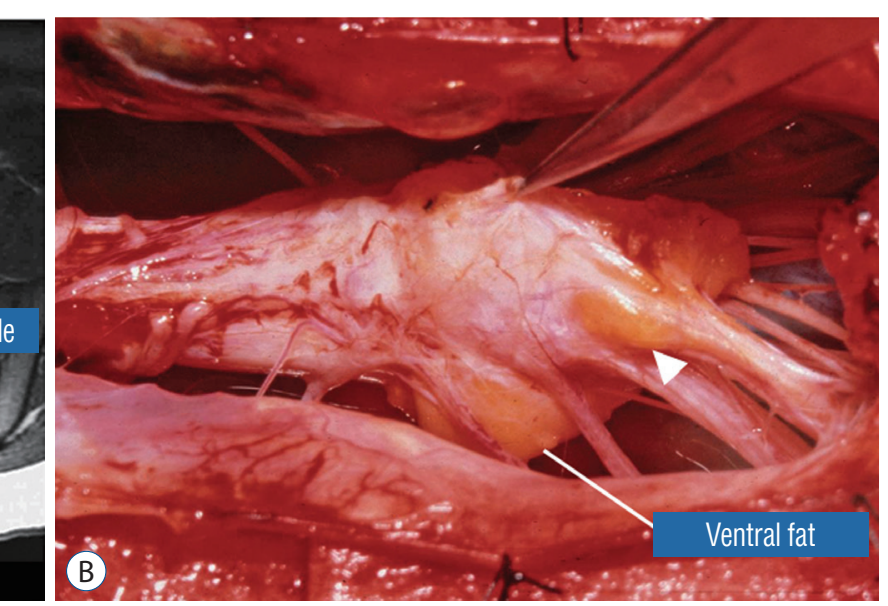

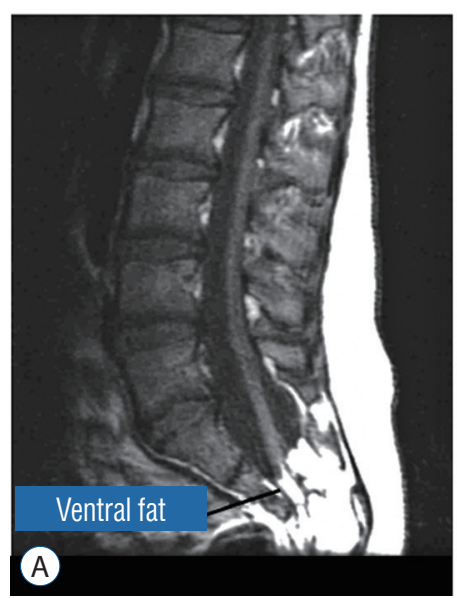

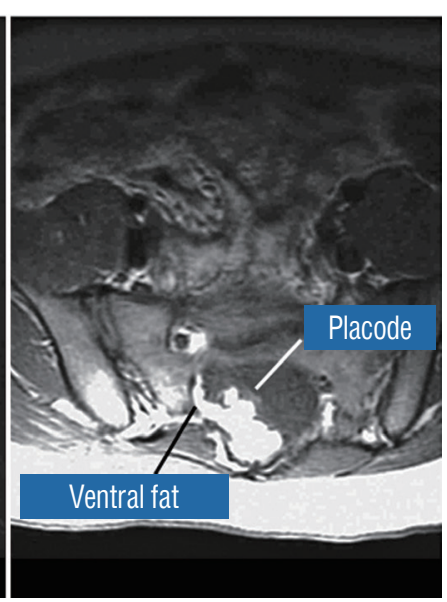

Fig. 4. Chaotic lipoma. A : Left : sagittal magnetic resonance imaging shows ventral as well as dorsal fat in relation to the neural placode. Note sacral agenesis with only two visible sacral segments. Right : axial image shows ventral fat and extremely irregular lipoma-fat interface. B : Intraoperative picture showing fat ventral to placode and on one of the sacral roots (arrowhead). Note absence of discrete fusion line. Reprinted from Pang et al. ${ }^{611}$ with permission from Springer Nature. 


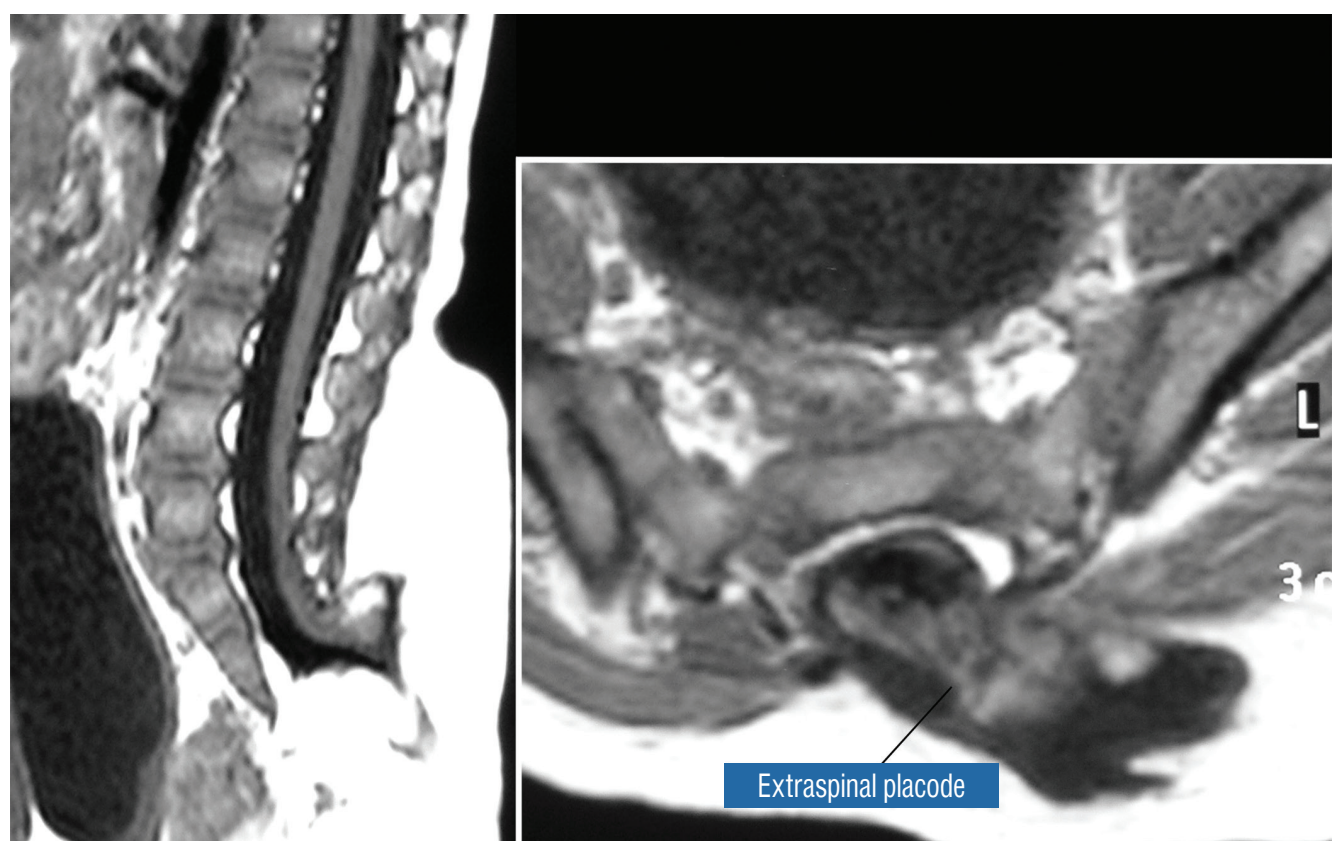

Fig. 5. Transitional lipoma with extraspinal extension ("lipomyelomeningocoele"), with the lipoma, cerebrospinal fluid sac, and part of the neural placode extending out of the spinal canal through a dorsal defect. Reprinted from Pang et al. ${ }^{611}$ with permission from Springer Nature.

is important because it guarantees exclusion of the extraneural mesenchyme from ever entering the neural tube to make contact with its inner (ependymal) surface. The embryologic error leading to the mesenchymal invasion of the sliding neural tube probably lies in premature disjunction between the two ectoderms ${ }^{7,43}$; i.e., ectodermal separation occurs before fusion of the converging neural folds, leaving a small gap next to the open neural tube through which paraxial mesenchyme can migrate into the central canal. Once such a mesenchymal stalk forms through this gap, further closure of the neural tube is prevented and a segmental dorsal myeloschisis is created (Fig. 6). Alternatively, the inversion of the fusion-disjunction sequence can also be caused by incompetence of the paraxial mesoderm in forcing timely convergence of the opposing neural folds, delaying their fusion and allowing disjunction to happen first ${ }^{30-36)}$. Lastly, metabolic disturbance of the cell membrane-bound glycosaminoglycans at the edges of the neural folds, which are vital to cell-cell recognition and adhe$\operatorname{sion}^{39,44,47,72)}$, could likewise delay neural folds fusion and reverse the sequence.

Pluripotential mesenchyme has been shown to form diverse derivatives depending on the kind of inducers secreted by the adjacent neuroectoderm ${ }^{13,24)}$. Once exposed to the invading mesenchyme, the ependymal lining of the neural tube has the inherent propensity to induce it to form fat, muscles, collagen, even bone and cartilage. In contrast, the outer surface of the neural tube normally initiates the production of meninges ${ }^{42)}$. However, new meninges cannot now form across the dorsal midline because of the impeding lipoma stalk in-evolution, which ultimately tethers the neural tube to the subcutaneous fat. Similarly, defects in the developing myofascial structures (from myotomal mesoderm) and neural arches (from scleromesoderm) also neatly surround the lipoma stalk (Fig. 6).

Once formed, the mesenchyme-derived fat and collagen within the neural tube fuse with the developing alar and basal plates. Because the dorsal root ganglions descend from neural crest cells pinched from the external surface of the neural folds just lateral to the site of failed fusion, their central processes, the dorsal nerve roots, also enter the spinal cord lateral to, but never traversing, the lipoma stalk. Their entry zone (DREZ) must correspondingly lie very near, but always lateral, to the exact junctional boundary between lipoma and spinal cord. Recognising and understanding this boundary, called fusion line, is essential before undertaking the actual resection of the lipo$\mathrm{ma}^{51,52,59)}$ (Fig. 6). Meanwhile, the "disjoined" cutaneous ectoderm crosses over the chaos underneath to form wholesome skin above, although minor perturbation in the process may produce subtle signatures such as a dimple, crater, or capillary 


\section{Embryogenesis of dorsal lipoma}

Premature disjunction

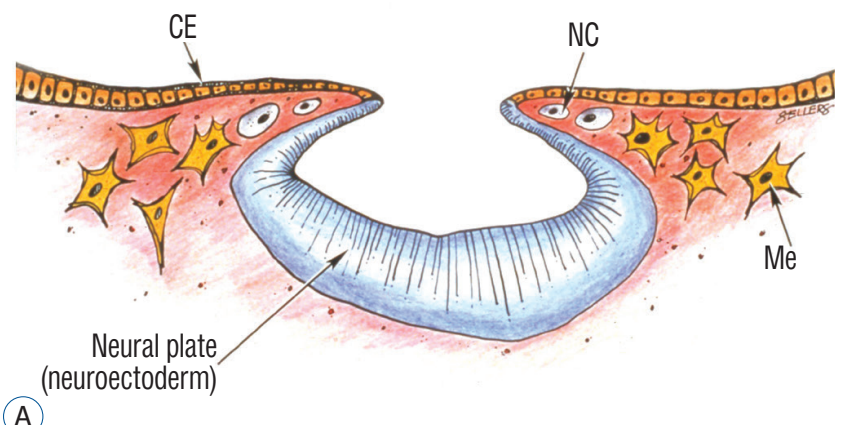

(A)

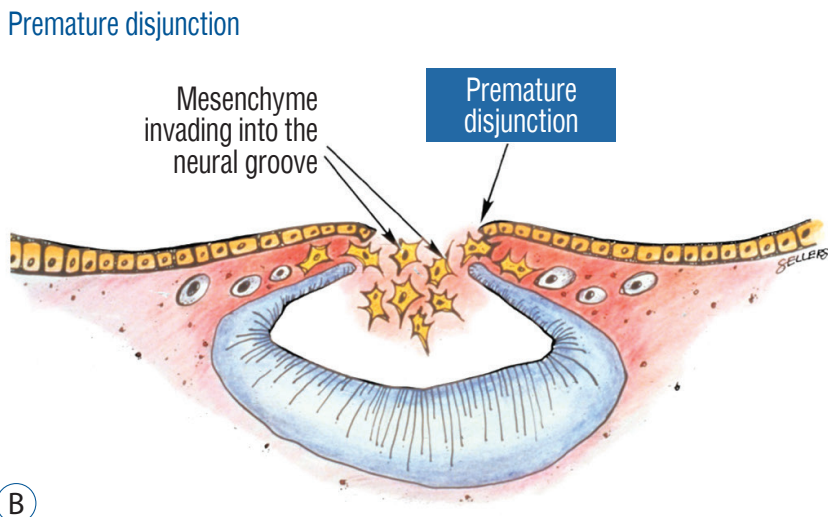

(C)
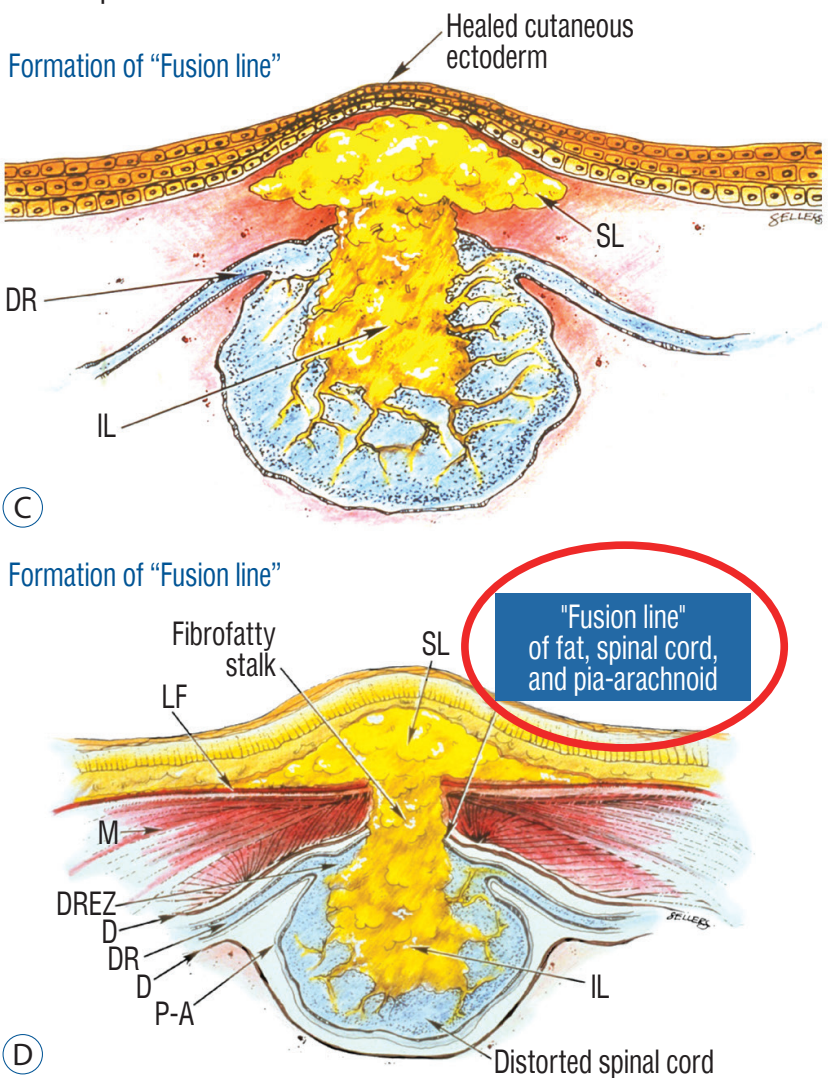

Fig. 6. Embryogenesis of dorsal lipoma, a pure primary neurulation defect. A and B : Premature disjunction before complete closure of neural plates allows migration of mesenchymal cells into neural groove to establish contact with the ependymal surface. C and D: Formation of fusion line between lipoma, cord, and pia-arachnoid. DREZ and dorsal root are always lateral to the fusion line and thus not entangled in fat. Reprinted from Pang ${ }^{55)}$ with permission from the Japanese Neurosurgical Society. CE : cutaneous ectoderm, NC: neural crest, Me : mesenchyme, SL : subcutaneous lipoma, DR: dorsal root, IL : intramedullary lipoma, DREZ: dorsal root entry zone, D: dura, P-A : pia-arachnoid, $\mathrm{M}$ : muscle, LF : lumbodorsal fascia.

haemangioma overlying the subcutaneous lipoma.

The embryogenesis of dorsal lipoma perfectly exemplifies mistimed disjunction during primary neurulation. Its fatty stalk only involves cord segments above the conus, the latter being product of secondary neurulation. Furthermore, premature disjunction of the converging neural folds is always segmental, meaning proper disjunction and closure take place "business-as-usual" both immediately rostral and caudal to the faulty focus. This "square pulse" time-line of the abnormal event in dorsal lipoma therefore results in a sharply demarcated fusion line both rostral and caudal to the lipomatous stalk ${ }^{51-54,59)}$ (Fig. 2A and B). Dorsal lipomas thus exist only on parts of the matured spinal cord formed from the primary neural tube, and represent only $15 \%$ of lipomas in our series $^{59-61)}$.

In transitional lipoma, faulty development involves much more than simple mistimed disjunction in an isolated segment of the primary neural tube. Even though its rostral part resembles the dorsal lipoma, the fatty invasion of the whole of the conus means that not only primary but also secondary neurulation have been profoundly disturbed. This is evidenced by the frequent incorporation of the filum, an undisputed remnant of late secondary neurulation, into the transitional lipoma, and that vacuoles resembling the cavitary spaces typically seen in the medullary cord during mid-secondary neurulation, are found in abundance within the core of the lipoma (see below). Also, while the rostral part of the transitional lipoma only affects the dorsal surface of the lumbar cord, the distal part involves the entire core of the conus, strongly suggestive of aberrant inclusion of wayward mesenchyme into the developing conus during the condensation phase of secondary neurulation. Intramedullary mesenchyme 
may then migrate up and down the neural tube within the conjoint primary and secondary neural canals ${ }^{66)}$. The hypothesis that the rostral half of the transitional lipoma comes from faulty primary neural tube fusion affecting only the superficial layers of the lumbar cord, and its caudal half from abnormal condensation within the deeper core of the secondary neural tube, may well explain the oblique ventral-caudal slant of its lipoma-cord interface. The far less confined and "nonsquare pulse" nature of its embryogenetic error also explains why the fusion line of a transitional lesion is never as well-defined and levelled as that of the dorsal lipoma, often has no caudal demarcation, and its fatty acreage is a great deal more sprawling. In spite of the latter, the lipoma-cord interface remains relatively distinct in most transitional lipomas.

\section{Embryogenesis of chaotic lipomas}

Chaotic lipoma does not quite fit into the scheme for either the dorsal or transitional type. It may have a rostral part halfresembling a dorsal lipoma, but caudally there is no definable lipoma-cord interface, and the fat percolates through the whole thickness of the neural placode to its ventral surface in large and unruly measures. The confusing interplay between fat and cord in this type of lipoma seems to be in constant chaos.

Embryologically, the obvious dissimilarities between the features of a chaotic lipoma and the familiar consequences of mistimed disjunction, as well as its strong association with sacral agenesis $\left(82 \%\right.$ in our 2009 series $\left.^{59}\right)$ ), suggest that its origin may be part of a general failure of the caudal cell mass during early secondary neurulation (Fig. 7$)^{45,46}$. Secondary neurulation comprises three distinct stages : 1) condensation and transformation of pluripotential stem cells from the caudal cell mass into committed neuro-progenitor cells to form the solid medullary cord; 2) intrachordal cavitation of the medullary $\operatorname{cord}^{46,65,66)}$ followed by its integration with the primary neural tube; and 3) partial dissolution of the cavitary medullary cord by programmed apoptosis to form the filum terminale ${ }^{28,66)}$. In this context, chaotic lipoma probably results from an aberrant preponderance and accelerated differentiation of lipogenic stem cells from the caudal cell mass during early medullary cord condensation, leading to inclusion of lipoid cells within the

- Severe disturbance of caudal cell mass during medullary condensation stage 1 of secondary neurulation

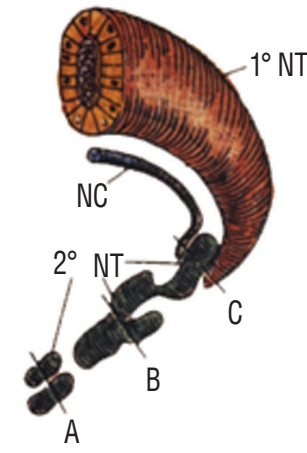

Stage 1: condensation of medullary cord
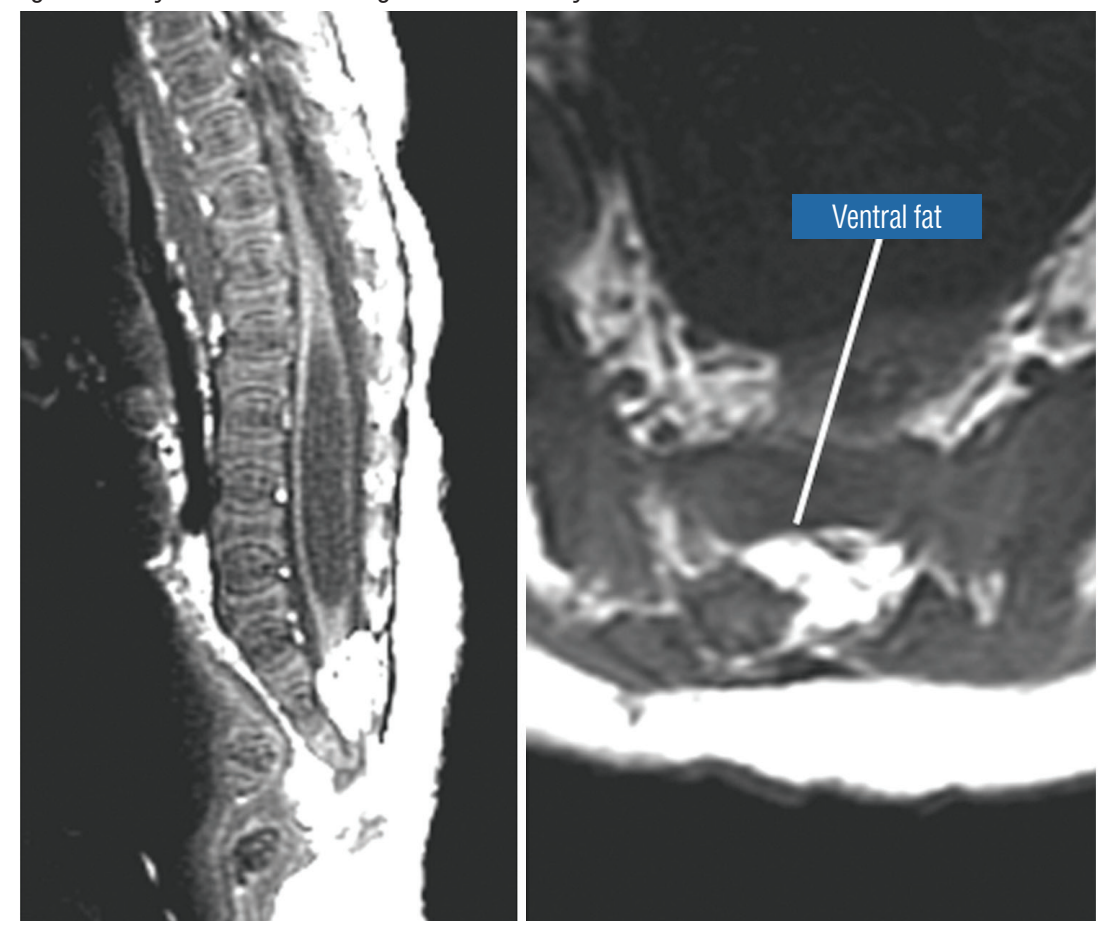

Fig. 7. Embryogenesis of chaotic lipomas. Left : basic error probably occurs with accelerated differentiation of lipogenic mesenchymal cells within the caudal cord during condensation stage (stage 1) of secondary neurulation and formation of the medullary cord, thereby incorporating fat tissue in the substance of the mature neural placode. Middle and right show dorsal and ventral fat and associated sacral agenesis. Reprinted from Pang et al. ${ }^{61)}$ with permission from Springer Nature. $1^{\circ} \mathrm{NT}$ : primary neural tube, $2^{\circ} \mathrm{NT}$ : secondary neural tube, NC: notochord. 
neuro-progenitor cell pool, destined to beget a distorted conus permeated with fat through and through ${ }^{599}$.

\section{Embryogenesis of terminal lipoma}

Terminal lipomas involve only the conus and never the lumbar or upper sacral cord, strong evidence that they arise from faulty secondary rather than primary neurulation. Furthermore, defects in the lumbodorsal fascia, dura, and dorsal spinal cord, all hallmarks of failed primary neural tube closure, are never seen with terminal lipomas. Lastly, terminal lipomas either replace or are embedded in portions of the filum, which temporally and topographically places their pathogenesis within the period of secondary neural tube formation. The fact that their distal conus always appears well-formed argues against disrupted condensation of the medullary cord in early secondary neurulation. Rather, the almost obligatory presence of disorganized neuroglial and ependymal tubules within terminal lipomas ${ }^{71}$ suggests instead an incompetent apoptotic machinery during the late degenerative stage of secondary neurulation as the basic pathogenetic mechanism ${ }^{58)}$.

\section{INTRAOPERATIVE ELECTROPHYSIOLOGICAL MONITORING}

Intraoperative electrophysiological monitoring is sine qua non in lipoma surgery $y^{49,50,56,59,611}$.

\section{Electromyographic needle placements}

A comprehensive electromyography (EMG) system is set up to capture triggered responses from muscles supplied by the lumbosacral nerve roots. Standard 27-gauge EMG needle electrodes are inserted into the rectus femorus $\left(\mathrm{L}_{4}\right)$, anterior tibialis $\left(\mathrm{L}_{4}-\mathrm{L}_{5}\right)$, gastrocnemius $\left(\mathrm{S}_{1}\right)$, and abductor hallucis $\left(\mathrm{S}_{2}\right)$. A pair of smaller gauge (No. 29) EMG needles are inserted obliquely into the external anal sphincter at the anal verge on each side. A plug of dry muslin gauze is pushed half way into the anal canal to isolate the sphincter contractions of one side from the other ${ }^{49)}$. In addition, EMG measurements are made from the abductor policis brevis in each hand as a monitor for equipment malfunction. All stimulations and EMG recordings in the author's unit are done with the Cadwell Cascade Intraoperative Monitoring System (Cadwell Laboratories, Inc, Kennewick, WA, USA) using the Cascade Software version 2.5, but several other system brands on the market are equally proficient.

\section{Bulbocavernosus reflex (BCR)}

This is the "electrical" version of the reflexive contraction of the external anal sphincter when the glans penis is firmly squeezed and quickly released. For the afferent sensory arm of the reflex, pairs of pad or needle electrodes are placed on the sides of the penile shaft in males and between the labia minora and the periclitoral skin in females. Stimulation of the somatic sensory domain of the pudendal nerve via these electrodes generates a reflexive contraction of the external anal sphincter measurable by EMG. BCR is therefore a form of $\mathrm{H}$ reflex within the conus useful in monitoring the integrity of the central sensorimotor connections of the sacral cord segments $^{49,50)}$.

Special comments need to be made about BCR. The pudendal somatic sensory - pudendal somatic motor arc, i.e., the basic BCR circuit, is bilateral, meaning that stimulation on one side will generate sphincter contractions on both sides. The response is highly stimulation-parameter dependent. We use a train of 4 stimuli to capitalise on the powerful principle of posttetanic potentiation, and fairly reliably obtain motor responses from the anus. However, BCR is highly sensitive to inhalation anaesthetic concentrations, so that a sevoflurane level of higher than $0.5 \mathrm{Mac}$ may disrupt it. The BCR is also uncertain in infants younger than 6 months so that allowances must be made for its inconstancy in newborn cases. But most of all, it must be clearly stipulated that the actual neuroelectrical circuit responsible for the BCR involves somatic sensory inputs from the perineal skin corresponding to the dermatomes of $S_{2}$ to $S_{4}$ via the pudendal sensory fibres, and somatic motor neurons within the anterior horn (Onuf's nucleus) projecting out via the pudendal motor fibres to the external anal sphincter. On the other hand, the sacral bladder circuit involves afferent visceral sensory fibres from the bladder wall stretch receptors via the pelvic nerve, and the efferent visceral motor neurons to the bladder detrusor muscles that reside within the autonomic (visceral) intermediolateral column of the central gray core of the sacral spinal cord, projecting out via the parasympathetic pelvic nerve. Thus, the anatomical pathways for the BCR and for the bladder voiding reflex are located in different, albeit neighbouring, parts of the spinal cord, though both are within the $\mathrm{S}_{2}-\mathrm{S}_{4}$ cord segments. Theoretically, a disrupted BCR during surgery does not necessarily imply damage to the bladder wall tension-detrusor reflex 
function, although frequently the prediction is true. A correlative study of the two is needed to sort out BCR's true value.

\section{Nerve root stimulation and direct spinal cord stimulation}

Triggered EMG is obtained from direct stimulation of the motor roots and spinal cord using the concentric coaxial bipolar stimulation probe with a small tip diameter of $1.75 \mathrm{~mm}$ (Medtronic Xomed, Inc., Jacksonville, FL, USA) (Fig. 8). The concentric configuration and small sizes of the anode-cathode rings permit delivery of extremely focused current to a very small target volume, ideal for selective activation of small and crowded electroresponsive units such as the delicate sacral rootlets and the compact anterior horn of the conus ${ }^{59}$. Stimulating currents from 0.3 to 5.0 miliamperes $(\mathrm{mA})$ are used depending on target impedence. Most functional motor roots can be triggered by currents of 0.3 to $1.0 \mathrm{~mA}$, though sometimes needing $1.5 \mathrm{~mA}$ if the roots are partially fibrotic or wrapped up in arachnoid adhesions. Direct spinal cord stimulation will require a current of $3.0 \mathrm{~mA}$ or higher, but seldom more than $6 \mathrm{~mA}$. Both sides will be recruited if the probe is placed on the midline, but biased ipsilateral response can be elicited if the probe is shifted to a point ventral to the equatorial plane of the cord's coronal aspect. The stimulation frequency is usually 10 per second. Noting the rythmic nature of the triggered contractions will distinguish them from the random spontaneous firing caused by surgical manipulation.

\section{Transcortical motor evoked potentials (TcMEP)}

In recent years, we have been able to reliably obtain intermittent monitoring of TcMEP from the external anal sphincter. Because transcortical stimulation unavoidably causes a general shudder of the patient's whole body, it is coordinated with the surgeon so that the surgical procedure can be momentarily halted. The surgeon usually asks for a round of stimulation right after a particularly delicate or risky part of the dissection is performed, or during a more casual phase of the procedure as a loosely-timed routine of monitoring. The stimulation parameters are the same as for the lower limbs: a train of 8 pulses, each with a duration of $75 \mu$ sec and intensity
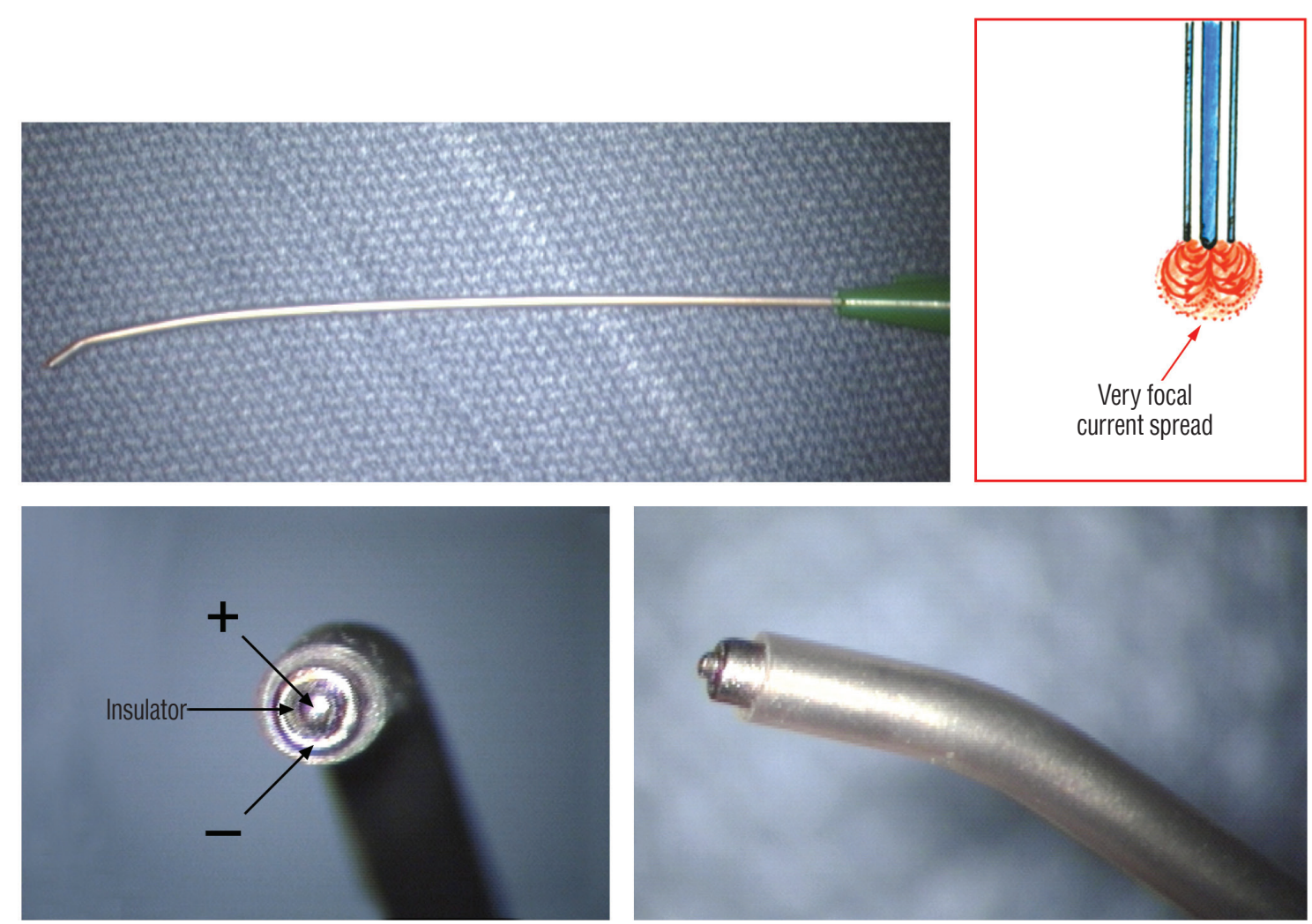

Fig. 8. Concentric coaxial bipolar microprobe stimulator in which the concentric cathode and anode are separated by a coaxial insulator. Tip diameter is approximately $1.75 \mathrm{~mm}$. The microprobe delivers very focal current spread (Inset). 
of 100-300 volts. In adults and children older than 2 years of age, 150 to 225 volts are generally adequate but in infants, the stimulation intensity may have to be raised to 350 volts, presumably because of the poorly myelinated motor cortex and its higher impedence, only partially compensated for by the thin cranium. The inhalation anaesthetic concentration, e.g., of sevoflurane, is usually set no higher than $0.5 \mathrm{Mac}$ to ensure a high response rate, or, failing that, abandoned completely for total intravenous anaesthesia (TIVA) in some infants and young children. Given that the BCR can be variable or even unobtainable in infants, TcMEP is an important modality for assessing the integrity of the central motor circuits of the sacral spinal cord.

\section{Somatosensory evoked potentials (SSEPs)}

Stimulating electrodes either of the pad or needle type are placed over the course of the posterior tibial nerve behind the medial maleolus and over the common peroneal nerve at the fibular neck to provide the somatic sensory inputs for SSEPs monitoring of the spinal cord segments above $\mathrm{S}_{2}{ }^{49}$ ) . It should be emphasized that the sensory inputs from both these nerves are going into the $\mathrm{L}_{5}-\mathrm{S}_{1}$ cord segments, considerably higher than the usual location of most lipomas, so disturbance of conduction in the $\mathrm{S}_{2}-\mathrm{S}_{4}$ sensory roots will therefore not affect the SSEPs.

\section{SURGICAL TECHNIQUE OF TOTAL/NEAR-TOTAL LIPOMA RESECTION}

The surgical technique described below concerns the case of an unusually large and rambling dorsal lipoma, that illustrates most of the key technical points of total resection applicable to both dorsal and transitional lipomas. The case is a 12 months old girl who was neurologically normal at birth but had magnetic resonance imaging (MRI) because of a lumbosacral fatty lump, which showed a moderate-sized dorsal lipoma involving the lower lumbar cord above a normal looking conus. At 1 year, she began showing left leg weakness and repeat MRI showed massive enlargement of the lipoma (Fig. 9).

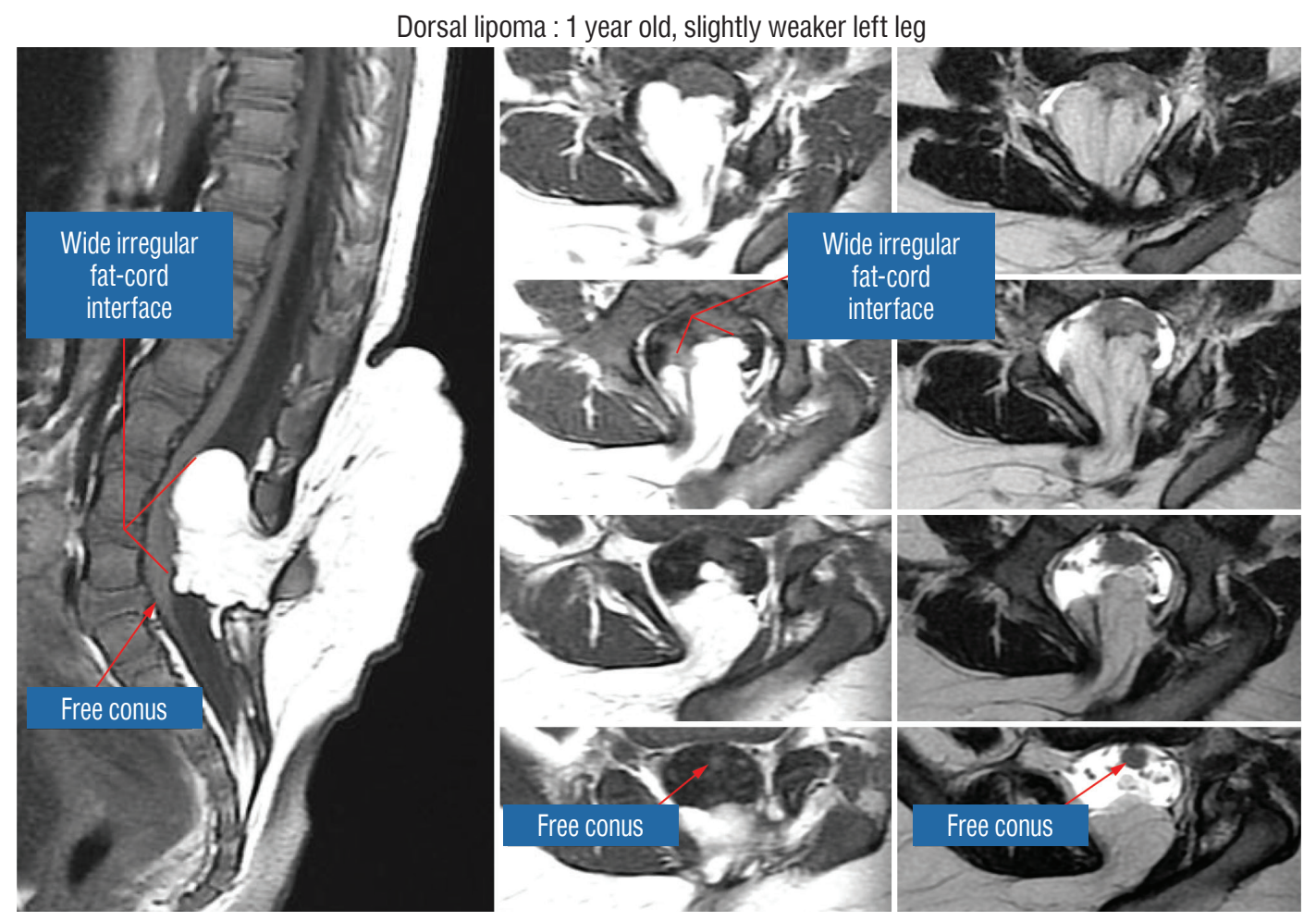

Fig. 9. Magnetic resonance imaging of a 12 months old girl with left leg weakness shows a large dorsal lipoma. The sagittal image shows a long stretch of lipomacord interface, but the conus is clearly free of fat. The axial images reveal the irregular nature of the lipoma-cord interface, but the neural placode is not excessively tilted to one side. Reprinted from Pang ${ }^{55)}$ with permission from the Japanese Neurosurgical Society. 


\section{Step 1. Exposure and dealing with the extraspinal lipoma stalk}

The upper extent of the skin incision should be about $2 \mathrm{~cm}$ above the rostral end of the lipoma. The lower extent ideally includes at least $1 \mathrm{~cm}$ beyond the tip of the conus in a dorsal lipoma or of the neural placode in a transitional lipoma. Temptation to remove the subcutaneous lipoma must be resisted to avoid creating a dead space which may become tensely fluid-filled and compromise wound healing. Frequently, a relatively robust fatty stalk more compact than the loose subcutaneous fat can be discretely traced through a defect in the lumbodorsal fascia with minimal dissection (Fig. 10). This fatty stalk is a signpost to the lipoma's attachment on the spinal cord below and must therefore be handled with care during fascial exposure.

The amount of proximal bone removal must be adequate to expose the rostral tip of the lipoma where the "last" set of normal nerve roots and DREZ are used as anatomical landmarks, but more importantly, wide bilateral laminectomy down to the pedicles must be done to gain access to the far lateral corners of the dural tube (see below). Exposure of some normal dura proximal to the lipoma also provides a visual perspective of how much the neural placode had protruded beyond the spinal canal in lipomas with extraspinal extension. The heavy mound of fat attached to the fatty stalk can now be drastically reduced to avoid inadvertent tugging on the spinal cord (Fig. 10).

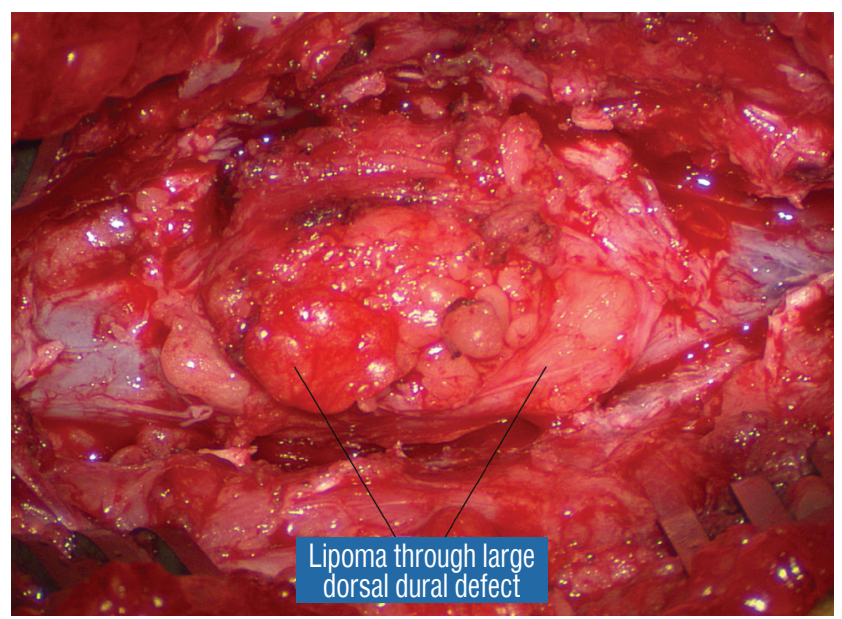

Fig. 10. Large extradural portion of the lipoma before dural opening. Note huge dural defect through which the extradural lipoma extends. Rostral exposure is to the right. Note the essential wide bony exposure. Reprinted from Pang ${ }^{55)}$ with permission from the Japanese Neurosurgical Society.

\section{Step 2. Detachment of lipoma from dura : un- hinging the hammock}

The dura is opened in the midline about $1 \mathrm{~cm}$ rostral to the upper end of the lipoma, identified by its slight yellow tinge below the pia. Tight dural tagging sutures are placed to gain maximum exposure of the far lateral alcoves of the dural tube afforded by the wide laminectomy. This is a crucial but often neglected manoeuvre, because tagging the dural edge helps to reveal the "crotch" at the embryological fusion line where the lateral fringe of the lipoma is attached to the inner edges of the dural defect. The critical importance of adequately exposing the "crotch" is shown best by examining any given axial slice of the lesion, in which the lipoma is roughly divided by a transverse line joining the site of lipoma-dura attachment, i.e., the "crotch", on each side. The entire lipoma-cord assembly is, in effect, suspended from the dural ceiling like a hammock at these two crotch hinges. From a dorsal perspective, the surgeon thus has no visualisation whatsoever of any meaningful anatomy ventral to this transverse line, such as the dorsal roots, DREZ, neural placode, or ventral CSF pool (Fig. 11 up-

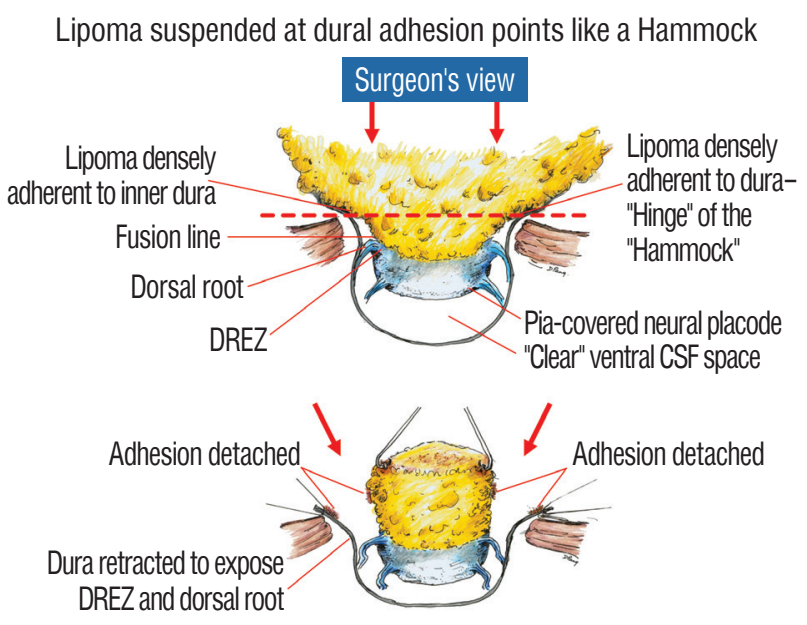

Fig. 11. Drawing depicting the relationship between the lipoma, neural placode, nerve roots and dural sac in an axial slice. Upper : the lipoma-cord assembly is suspended at the dural edges at far lateral adhesion points like a hammock against side hinges. The dotted transverse line that joins the two side hinges divides the assembly into a dorsal disorderly, fibrofatty half that completely blocks the surgeon's view to a much more orderly ventral half, containing the important anatomical landmarks of fusion line, DREZ, dorsal roots, fat-free ventral placode, and pristine ventral CSF space. Lower : after detaching the far lateral adhesion points (the hinges) by careful "crotch dissection", and folding-in the fatty mass, the ventral anatomical landmarks can now be visualized. Reprinted from Pang ${ }^{55}$ with permission from the Japanese Neurosurgical Society. DREZ : dorsal root entry zone, CSF : cerebrospinal fluid. 
per), until this hammock can be unsuspended by releasing the two crotch hinges. The lateral edges of the neural placode and nerve roots can then be folded inward enough to be identified and preserved (Fig. 11 lower).

Safe unhinging of the hammock must begin at the rostral extent of the crotch line where there is at least a semblance of normal anatomy. The "crotch" is kept under tension by pulling the fat firmly away from the dura, and its attachment to the inner dural lining minutely cut literally millimeter at a time to avoid injuring the dorsal roots on the DREZ, which are very close to the "crotch" only millimeters below the fat

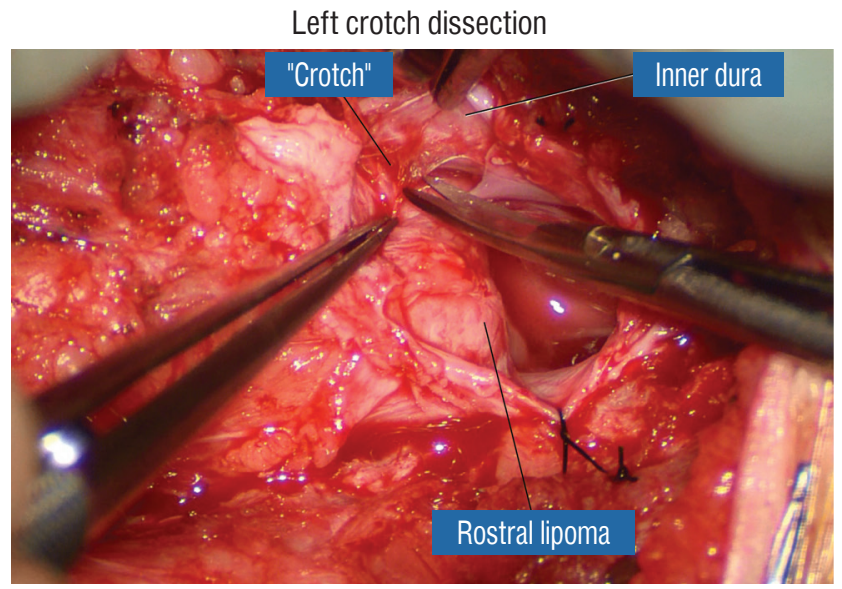

Fig. 12. Left crotch dissection in the large dorsal lipoma shown in Fig. 10. The lipoma is grasped firmly and pulled gently away from the adherent points on the inner dura, stretching the adhesion bands and thick arachnoid, creating the "crotch" and making the adhesions safe to be cut. Reprinted from Pang ${ }^{55)}$ with permission from the Japanese Neurosurgical Society.
(Fig. 12). These initially hidden roots should "pop" into view as more of the fatty hammock is peeled back (Fig. 13). The clean ventral surface of the neural placode is now seen to advantage. (Fig. 14). This labourious but indispensable and ultimately rewarding step of "crotch dissection" is carried caudally until all the functioning nerve roots are revealed and the entire lipoma-neural placode assembly is completely unsuspended and freed from the dura (Fig. 15).

\section{Step 3. Lipoma resection}

Before the actual lipoma resection, the fusion line must first

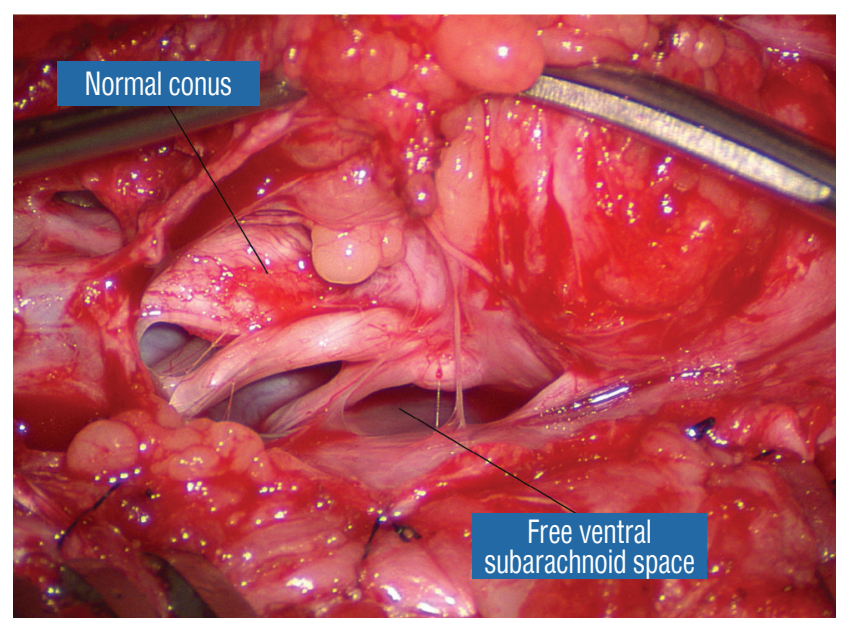

Fig. 14. After crotch dissection on the right side and complete detachment of the lipoma (hammock) from the right inner dural lining, the fat-free ventral subarachnoid space, caudal nerve roots, and conus are well seen. Reprinted from Pang ${ }^{55)}$ with permission from the Japanese Neurosurgical Society.
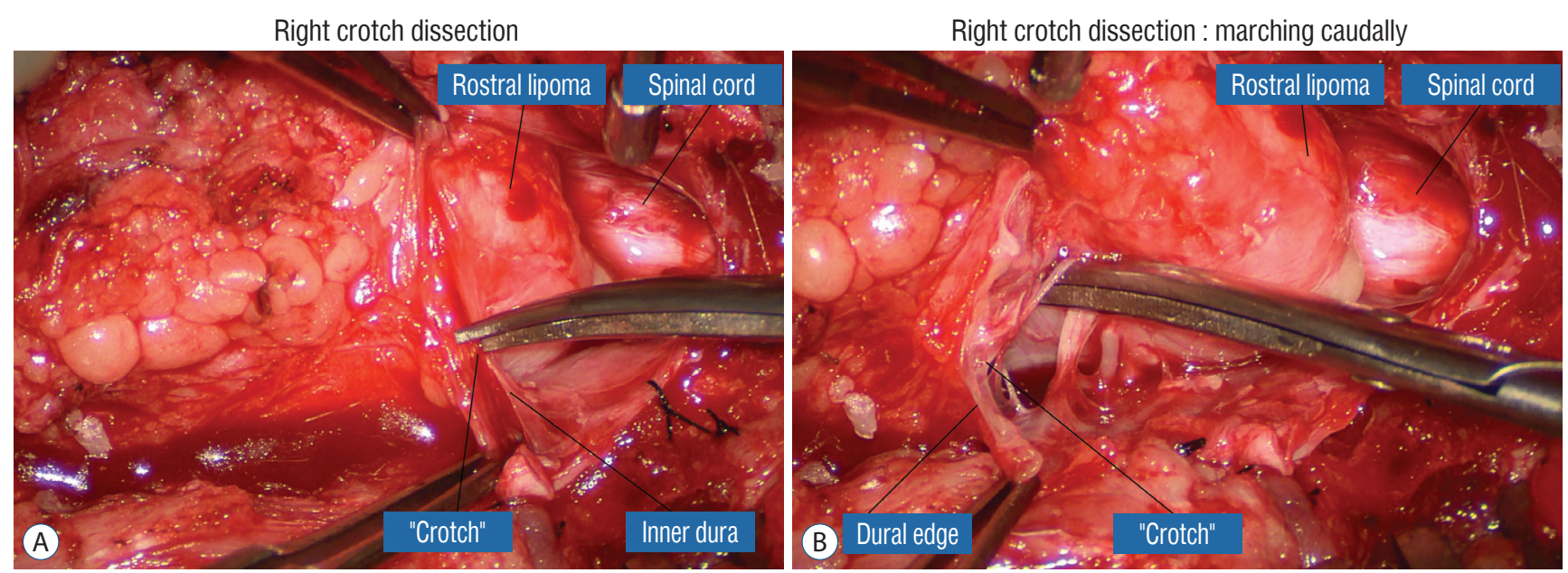

Fig. 13. Right crotch dissection. A : Cutting of the "crotch" on the right side as in Fig. 12. B : Lysing the crotch exposes the hidden nerve roots, the ventral neural placode, and the ventral free subarachnoid space. Reprinted from Pang ${ }^{55}$ with permission from the Japanese Neurosurgical Society. 
be clearly identified because a good amount of sharp dissection of fat will fall on this line where the lateral edge of the lipoma is attached to the cord just medial to the DREZ. In most dorsal lipomas, the fusion line is traceable as a neat complete oval or circle from side to side, often outlining a flat horizontal plane, and is usually bilaterally symmetrical. This circular line ends short of the conus which is never involved in the dorsal lipoma (Fig. 2B). In a transitional lipoma, the more distinct rostral fusion line resembles that of a dorsal lipoma but distally it becomes blurry and cuts towards the ventral surface

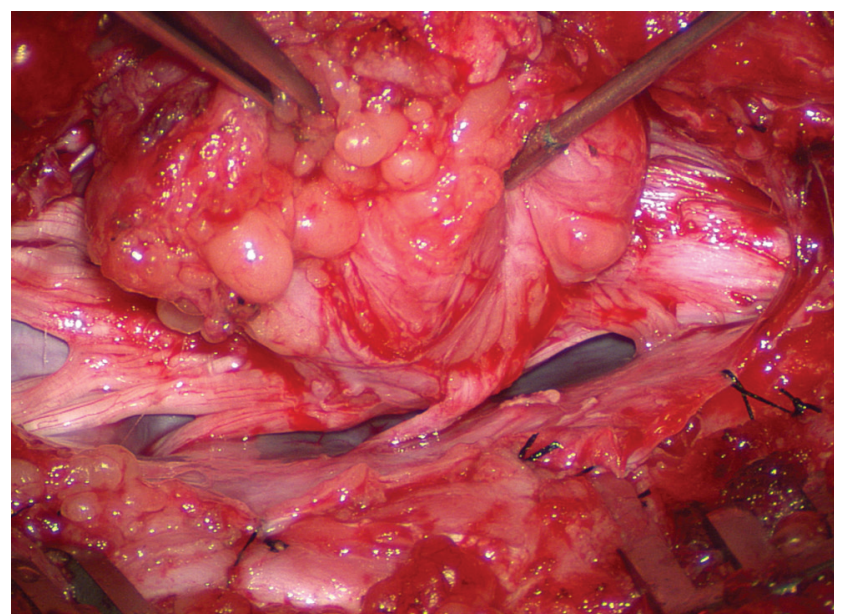

Fig. 15. Crotch dissection is complete on both sides. The entire right array of nerve roots are seen, but not the dorsal root entry zone or the fusion line, which are covered by the lateral overhang of the large lipoma. The entire hammock is now unsuspended from the dura. Reprinted from Pang ${ }^{55}$ with permission from the Japanese Neurosurgical Society.

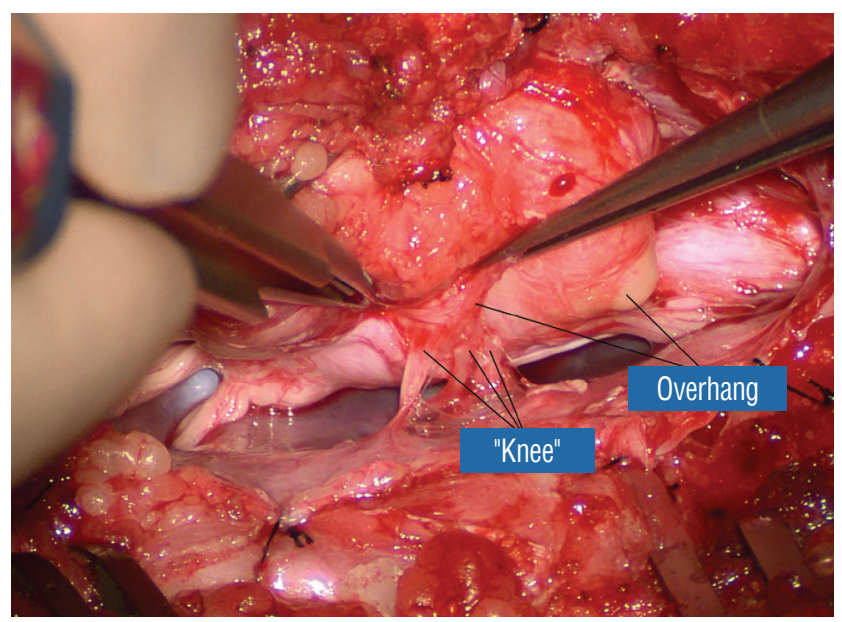

Fig. 16. The lateral overhang of the lipoma is well appreciated. Only the "knees" of the dorsal roots are seen. The "thighs", or the most proximal portions of the roots, are hidden by and adherent to the overhanging fat. Reprinted from Pang ${ }^{55}$ with permission from the Japanese Neurosurgical Society. of the conus, and never joins its mate from the other side at the bottom of the spinal cord (Fig. 3A and B). For a large lipoma of either type, as in the monstrous dorsal lipoma shown in Figs. 9 and 16, exuberrant fat may hang over the fusion line and cover up the upper portion of the emergent dorsal roots (the "thighs" of the roots) to give the false impression that the roots run through and are not separate from the lipoma (Fig. 16). In reality, these festoons of overhanging fat can quite easily be sharply dissected off the "thighs" of the roots to expose the "knees" of the roots (Fig. 17), the DREZ (Fig. 18), and the

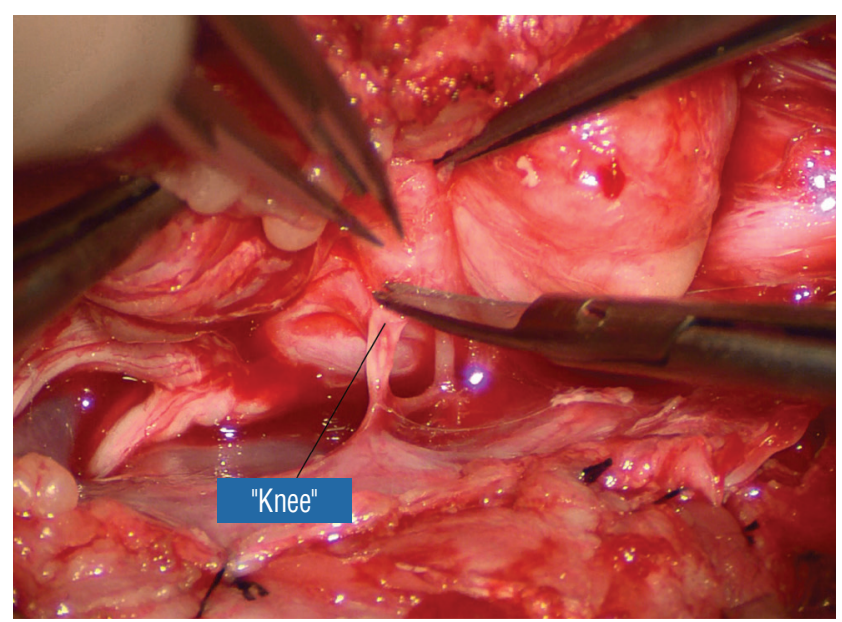

Fig. 17. "Knee dissection": the adhesions covering the "thigh" of the dorsal roots are sharply taken down. Reprinted from Pang ${ }^{55)}$ with permission from the Japanese Neurosurgical Society.

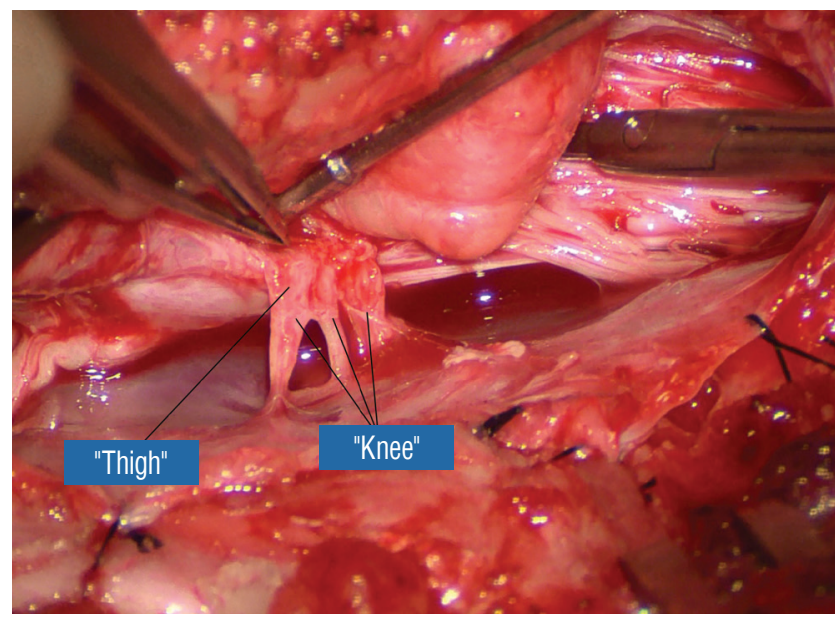

Fig. 18. After ridding the adhesions, the proximal "thigh" portions of the dorsal roots are exposed. Reprinted from Pang ${ }^{55)}$ with permission from the Japanese Neurosurgical Society. 
elusive fusion line above (Fig. 19). I call this manoeuvre "knee dissection".

Actual lipoma resection may commence only after the fusion lines from both sides have been clearly identified. Without exception, resection begins at the rostral tip of the lipoma where the anatomical relationships between fat, nerve roots, and DREZ are clearly decipherable (Fig. 20). First order of business is to use the micro-scissors to locate a thin but distinct silvery white plane between fat and cord at the demi-lune of the rostral fusion line (Fig. 21A). The white plane is in essence a thin net of collagen fibers that is distinguishable from yellow fat by its glistening white colour, and from soft spinal cord by its tough and gritty feel when cut. It can become convoluted and uneven within the main bulk of the lipoma, but its rostral end is almost always distinct and flat (Fig. 21B). The initial sharp cut to locate the plane has to be directed seem-

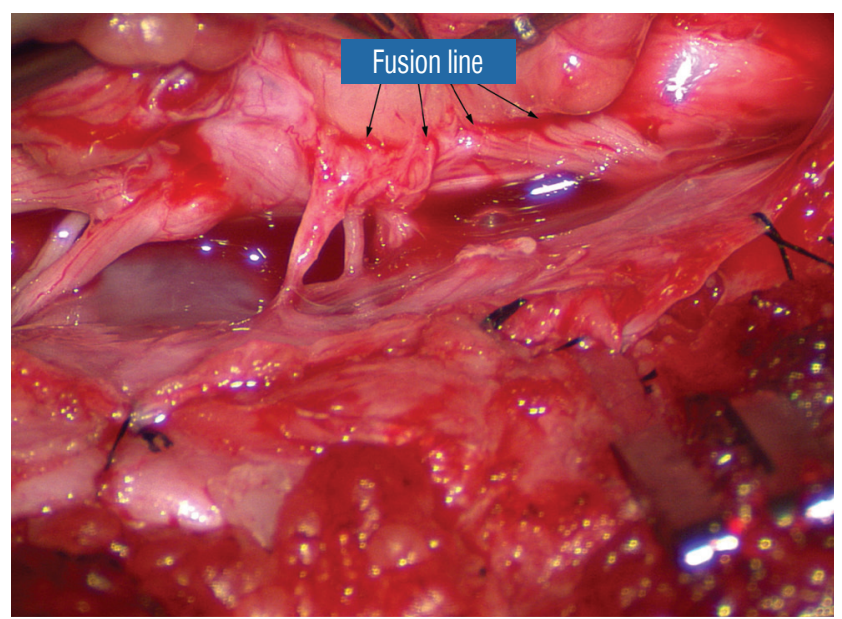

Fig. 19. The "true" fusion line on the right is revealed. Resection of lipoma on the lateral margin of the placode is now made safe. Reprinted from Pang ${ }^{55)}$ with permission from the Japanese Neurosurgical Society. ingly straight into the spinal cord but after neatly cutting through the tongue of yellow fat at the rostral tip of the lipoma, the gritty white plane can always be safely located here, by colour or by the feel of the scissors (Fig. 21C). Once found, it can then be followed by constantly sensing the grittiness through the micro-scissors and by noting the white glint between yellow globular fat and the dull, pasty pink spinal cord (Fig. 22). The $\mathrm{CO}_{2}$ laser should never be used to vapourise the fat because it chars the surface and thus blots out the signature white colour of the white plane, and also casts out the valuable tactile feedback from the micro-scissors. The Nd-YAG laser has less charring effect on the fat but without the tactile feedback, is no better in finding or surfing the white plane

Bleeding on the white plane, which is essentially bleeding on the cord, must be handled delicately. The exact bleeding spot is accurately and atraumatically located by intermittent

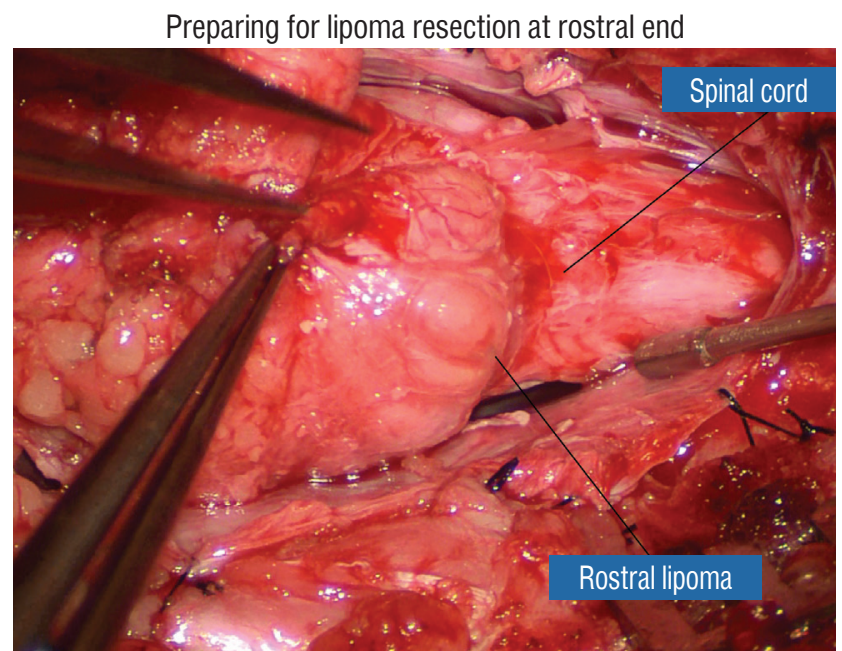

Fig. 20. Preparing for lipoma resection commencing on the rostral end of the fat where the lipoma-cord junction, the rostral dorsal roots, and beginning of the fusion lines are most distinct. Reprinted from Pang ${ }^{55}$ with permission from the Japanese Neurosurgical Society.
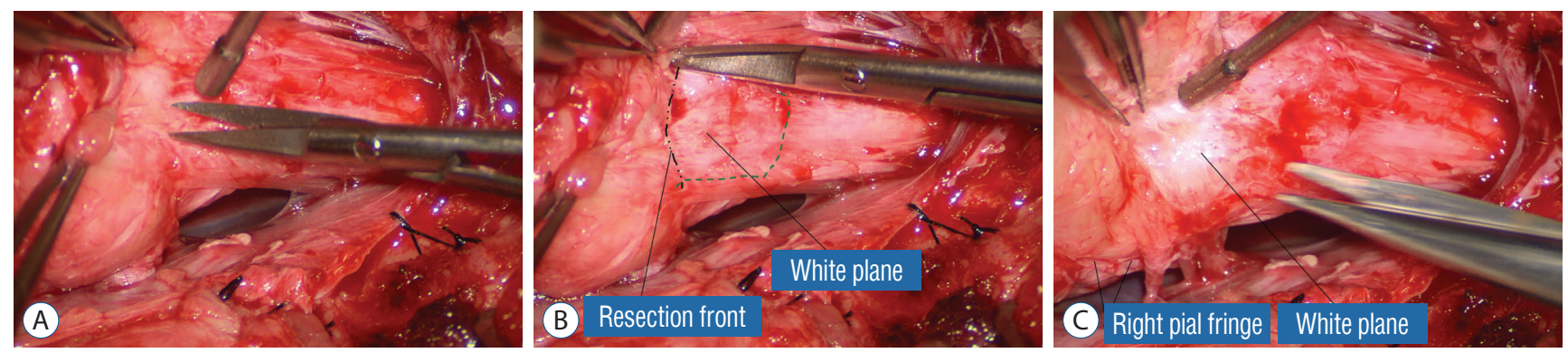

Fig. 21. Finding the white plane. A : Beginning resection at the rostral extremity of the lipoma. B: White plane located, which is a discrete though thin layer of whitish fibrous netting separating fat from spinal cord. C: Resection of lipoma along the right fusion line. Note the pial fringe, carefully preserved for neurulation. Reprinted from Pang ${ }^{55}$ with permission from the Japanese Neurosurgical Society. 
gentle jets of irrigation from a small hand bubble-squeezer fitted with a fine blunt needle. Once precisely located by the irrigation, the bleeding can be deftly handled with the ultra-fine irrigating bipolar cautery (0.2 mm tips; Fig. 23) set at a low current. The cold irrigation prevents sticking but more importantly it dissipates heat rapidly from the cord.

While resecting lipoma along the fusion lines (Fig. 24), strong diathermy and sharp gouging must be avoided at the DREZ to avert disturbing sensory symptoms. Just medial to the DREZ at the fusion line, a robust cuff of pia mater must be preserved to provide sturdy stitch-hold for the neurulation su-

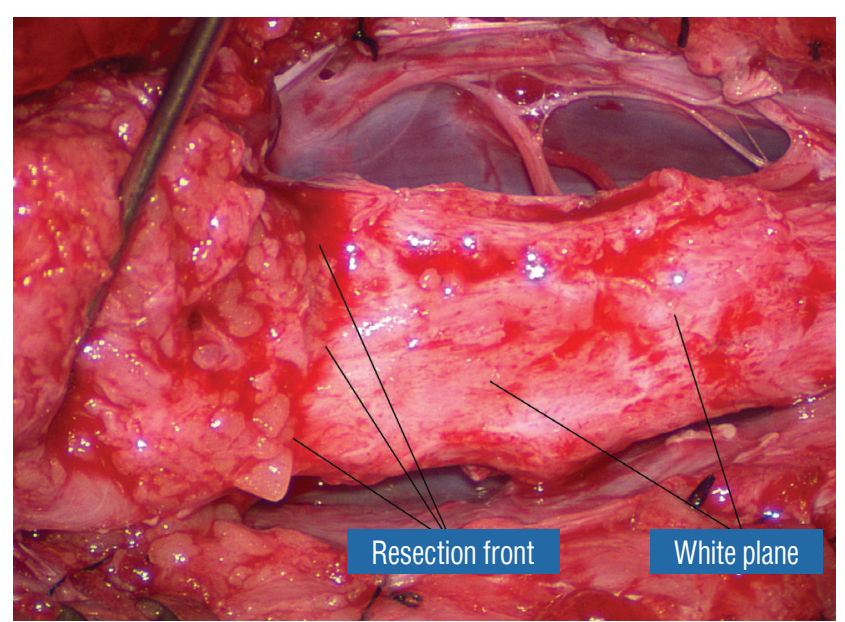

Fig. 22. The white plane, with the unresected portion of the lipoma on the left lifted up to show the resection front as a well defined transverse line across the body of the neural placode. Reprinted from Pang ${ }^{55}$ with permission from the Japanese Neurosurgical Society.

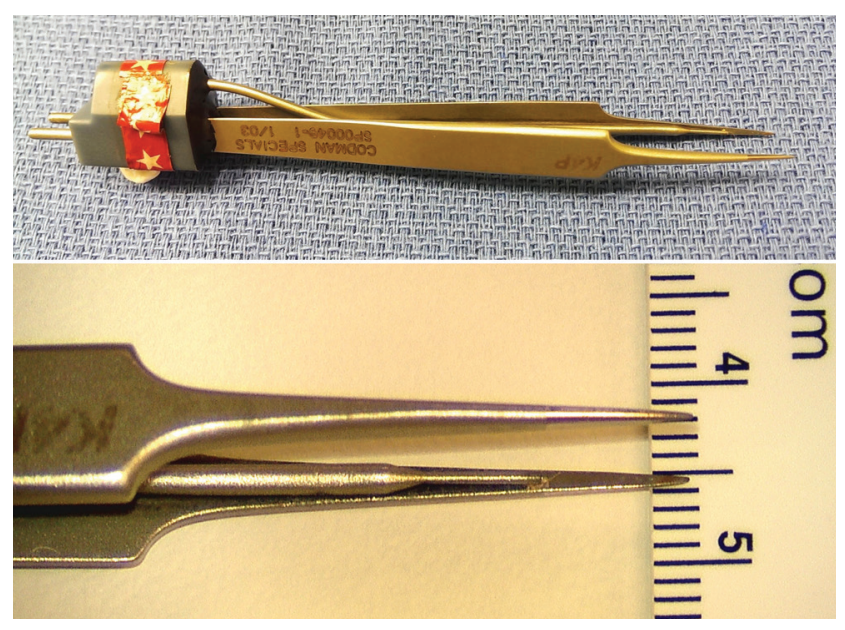

Fig. 23. Micro-irrigating bipolar cautery with super-fine tips measuring less than $0.2 \mathrm{~mm}$. Reprinted from Pang ${ }^{55)}$ with permission from the Japanese Neurosurgical Society. tures (see below) (Figs. 21C and 22). As long as the sharp dissection is kept strictly on the white plane medial to the fusion line and DREZ, complete lipoma resection can be carried out without injury to neural tissues. This is relatively easy to achieve in most dorsal lipomas because the white plane is usually predictably flat and levelled, and because the entire fusion line can be readily charted and circumferentially approached from all $360^{\circ}$ (Fig. 25), but in a large and rambling dorsal lipoma, the white plane may undulate considerably so that the corresponding fusion line and its neighbouring DREZ will accordingly situate at uneven heights on the side of the cord. Finding this safe zone of dissection may require systematic stimulation of the adjacent nerve roots for functional localization.

Navigating the white plane in most transitional lipomas is considerably more challenging because the plane is seldom horizontal, usually undulates, and the corresponding fusion line is thus very jagged. One side of the white plane with the DREZ and nerve roots may even be tilted completely away from the surgeon when the entire neural placode is rotated $90^{\circ}$ into the sagittal plane. In such extreme cases, the neural placode now faces one side of the canal and the lipoma the opposite side and the white plane is now vertical. Not uncommonly, the caudal portion of the lipoma is so large as to obscure all edges of the caudal dural sac, and one often encounters white bands that could be vestigial or functional nerves. Here, electrophysiological identification of "live" ventral nerve roots is the only way to determine where functional conus joins the

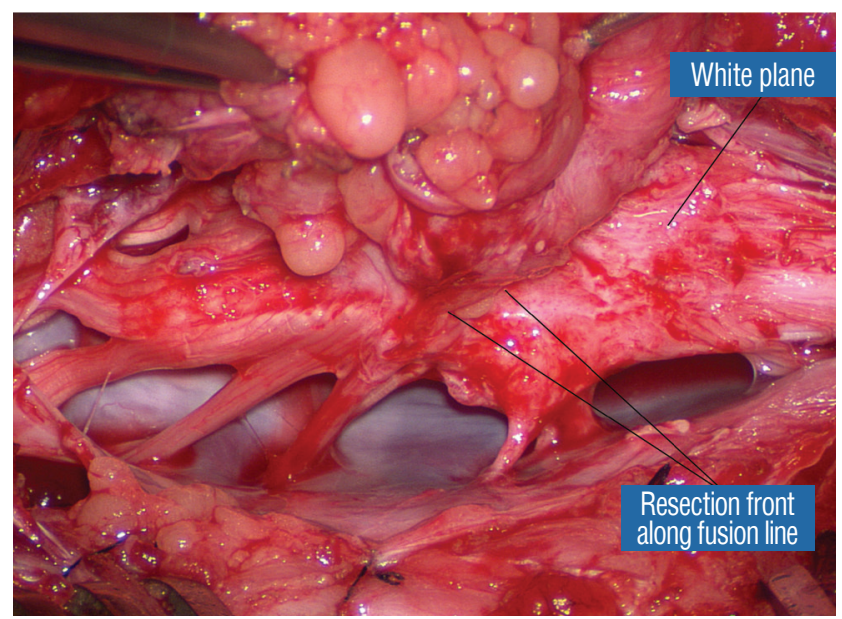

Fig. 24. Lateral white plane dissection along the right fusion line is almost complete. Reprinted from Pang ${ }^{55)}$ with permission from the Japanese Neurosurgical Society. 

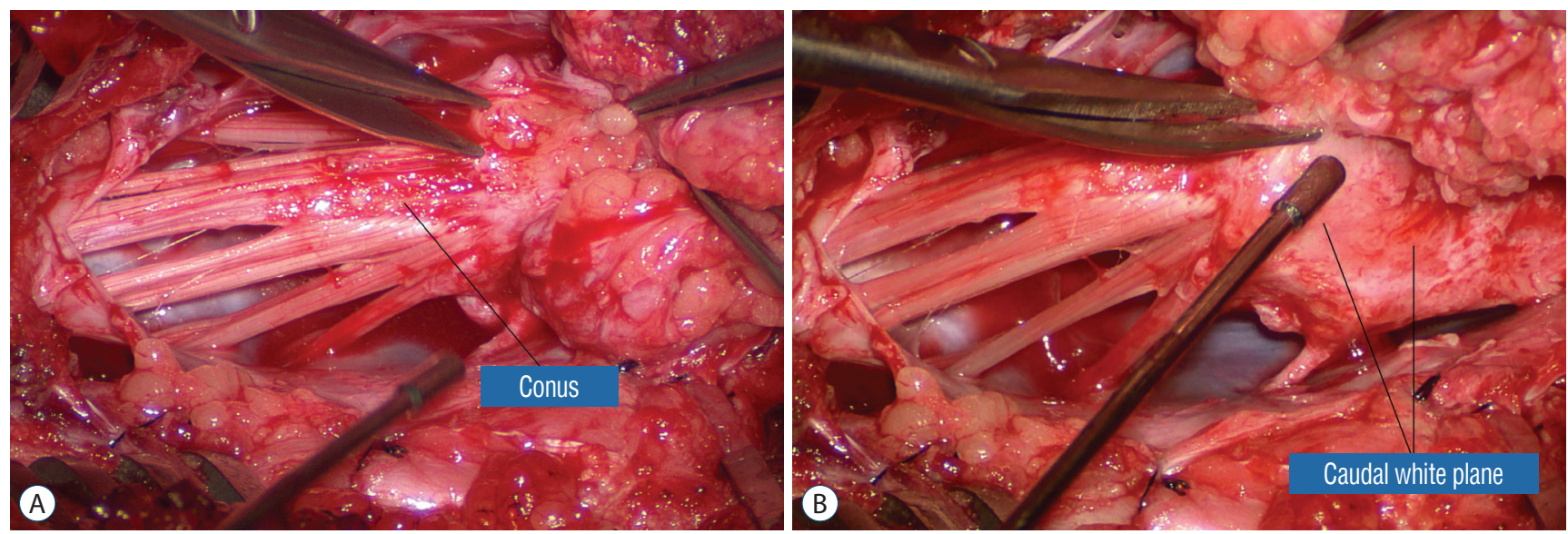

Fig. 25. Resection of the most caudal portion of the lipoma in the caudal to rostral direction, possible because this is a dorsal lipoma. The caudal white plane thus created will eventually merge with the proximal white plane resulting from the previous rostral-to-caudal dissection. A : Beginning white plane dissection from the conus side. B : More caudal white plane exposed. Reprinted from Pang ${ }^{55}$ with permission from the Japanese Neurosurgical Society.
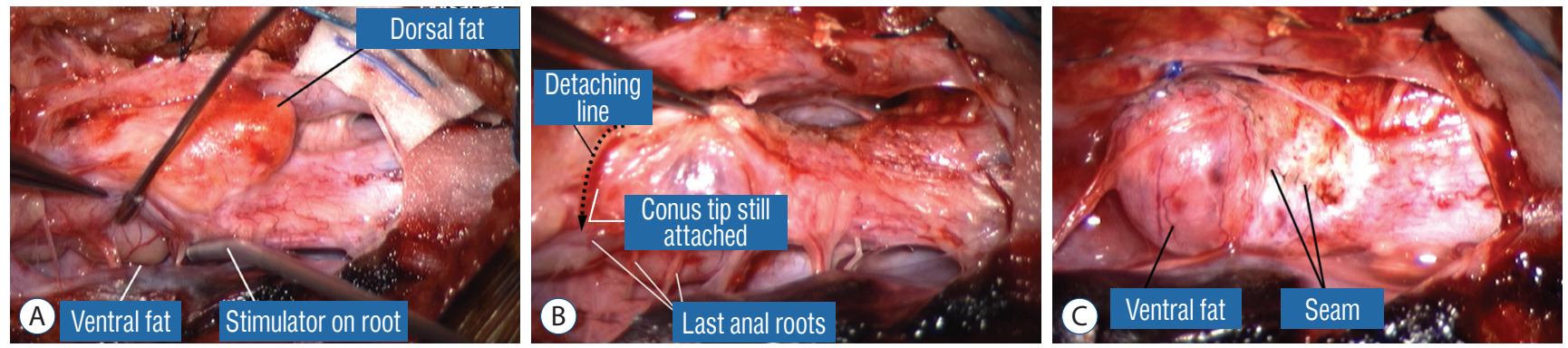

Pre-operative

Post-operative
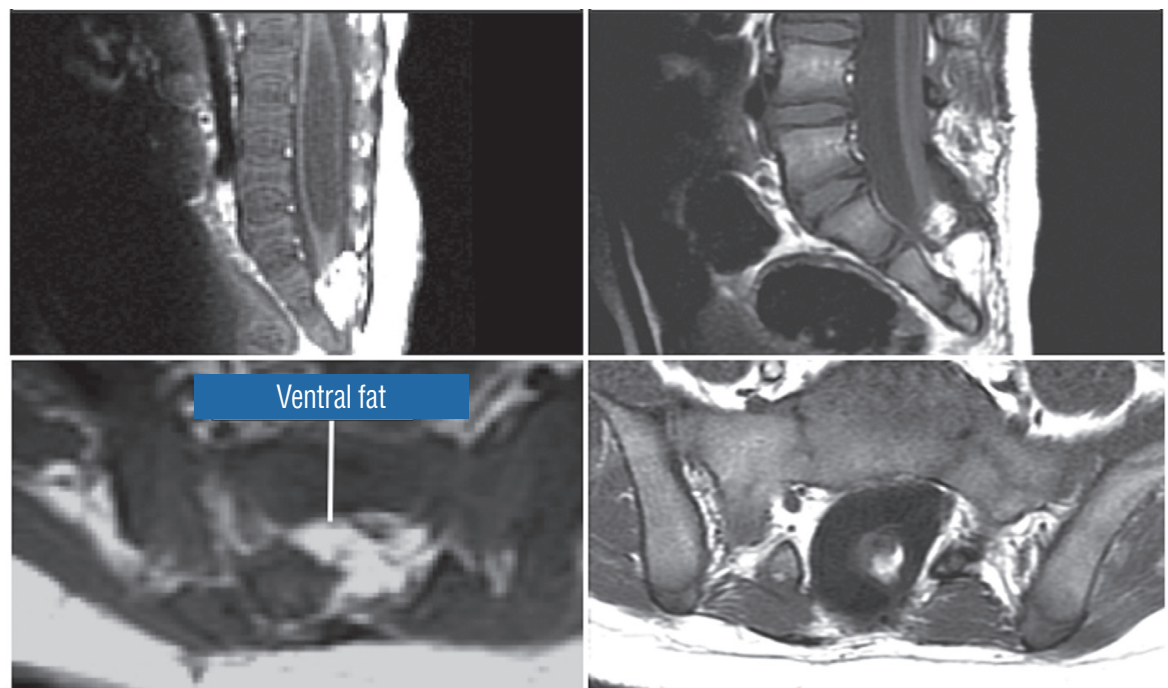

(D)

Fig. 26. Surgery for chaotic lipoma. A : Note ventral pia-covered fat medial to ventral nerve roots (being stimulated by concentric microprobe stimulator), and dorsal fat perched on the dorsal side of the placode. B : Terminal disconnection of neural placode from residual caudal lipoma stump after identification of 3 healthy pairs of anal sphincter motor roots. C: Caudal placode pulled up dorsally width-wise to be neurulated with the more proximal pial edge to form the seam, displaying the unviolated pia-covered ventral fat as a blunt stump. D : Pre- and postoperative magnetic resonance imaging shows residual fat on the detached cord stump. The cord is untethered, the thecal sac is augmented, and the syrinx has collapsed. A-C are reprinted from Pang ${ }^{55)}$ with permission from the Japanese Neurosurgical Society. D is reprinted from Pang et al. ${ }^{611}$ with permission from Springer Nature. 
fibrofatty muck of the caudal lipoma. The simple rules are as follows : $S_{2}$ is the only motor root that activates both the anal sphincter and the abductor hallucis, and the next two sets of puny and adhesion-laden rootlets with "pure" sphincter response must therefore be the $S_{3}$ and $S_{4}$ roots. Any fibrofatty muck or bands distal to the last "live" sphincter roots can now be considered non-functional and be freely discarded to consummate the final emancipation of the neural placode (Fig. 26B).

The same principle of using electrical identification of the caudal roots and placode may well be the only way to sort out

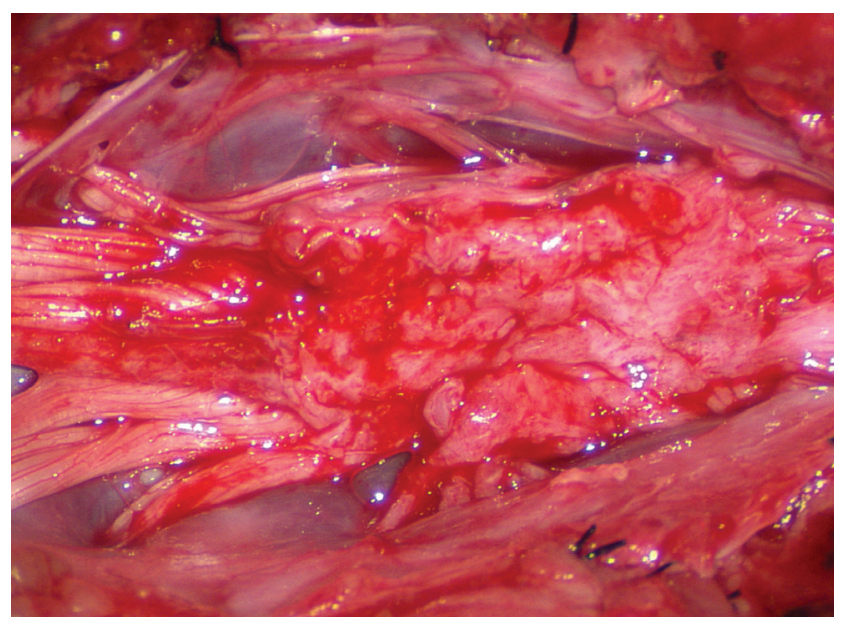

Fig. 27. Continuing from Fig. 25B, the "naked" neural placode after complete resection of the dorsal lipoma. Reprinted from Pang ${ }^{55)}$ with permission from the Japanese Neurosurgical Society.

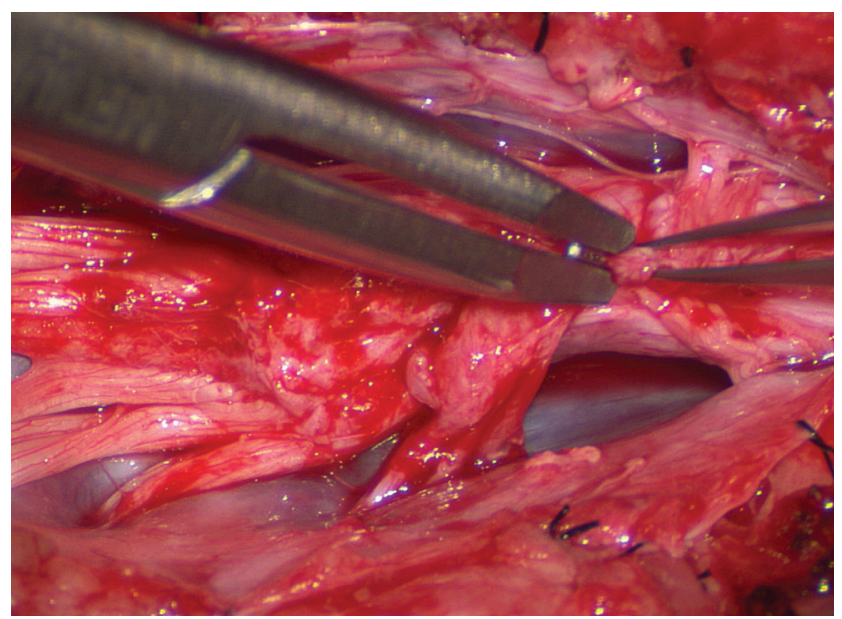

Fig. 28. Temporary small Weck Clips are applied to the apposed pial fringes to absorb the tissue torque tending to unfurl the dorsal bending of the placode. Reprinted from Pang ${ }^{55)}$ with permission from the Japanese Neurosurgical Society. the true termination of the functional conus in chaotic lipomas. With placode stimulation, the current is raised up to 4-6 $\mathrm{mA}$, but the drill is the same as for the motor roots. For the rostro-dorsal portion of the chaotic lipoma, dissection of the white plane is as described for dorsal lipomas, but the billows of fat ventral to the placode should be left alone and their pristine pial surfaces left unviolated (Fig. 26). It is always the dorsal and never the ventral surface of an untouched lipoma that actually tethers the spinal $\operatorname{cord}^{57)}$.

After the entire lipoma has been resected (Fig. 27), it is advisable to systematically stimulate each pair of motor roots on the "naked" placode and to run a few sets of transcortical motor potentials to ensure all baseline functions have been preserved. Functional deterioration has been known to occur during neurulation due to tight strangling of the placode.

\section{Step 4. Pia-to-Pia neurulation of the neural plac- ode}

Thorough resection of fat and fibrous scar converts a stiff, rotund, fat-engorged spinal cord to a supple, slender, and infinitely more manoeverable neural placode suited for tensionless pia-to-pia neurulation (Fig. 27). Well-executed neurulation in turn transforms the resection bed from a broad, wafery, sticky flat surface to a single seam on a trim and tidy pia-covered tube, duly minimising the likelihood of its adhering to the dura.

A few technical tips concerning neurulation deserve special

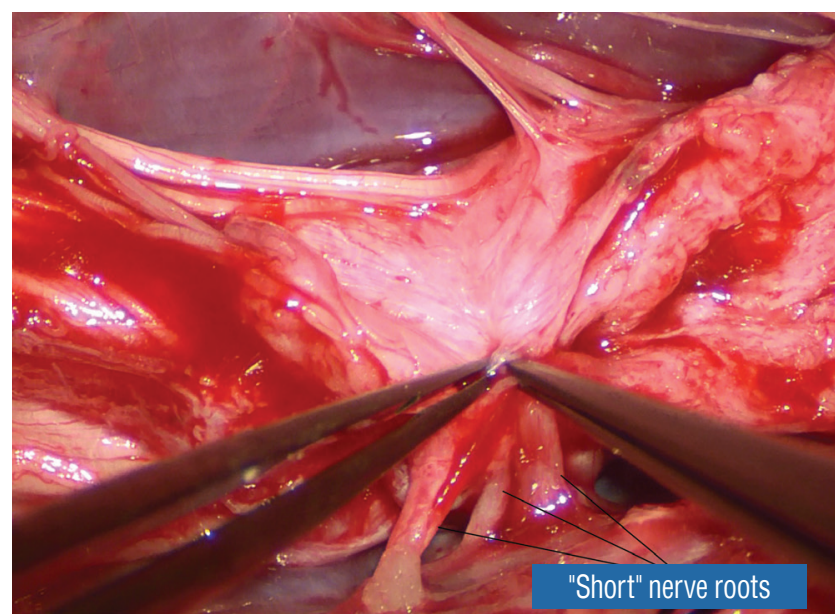

Fig. 29. On pulling the pial fringes together to check the ease of the dorsal neurulation, considerable lateral tugging is felt on the right, due to the "short" nerve roots. Reprinted from Pang ${ }^{55)}$ with permission from the Japanese Neurosurgical Society. 
mention. While folding up the neural placode to appose the pial edges, the surgeon may encounter a substantial torque tending to unfurl the folding due to the natural inelastic property of neural tissue. This can be mitigated by applying small Weck clips on the apposed pial cuffs at short intervals along the seam (Fig. 28), before sewing in the 8-0 nylon pial sutures in between the clips, always with buried knots. The Weck clips are then removed after enough sutures have been put in.

Occasionally, strong tugging on the inbending folds prevents their easy coaptation, caused by nerve roots that are trapped by adhesion bands on their underside (Fig. 29). These "short" and presumed to be non-functional and "fibrotic" roots are often found to be pliant and perfectly functional on electrical stimulation but merely bound up by adhesion bands. Cutting these bands releases the roots from the dura (Fig. 30), and duly "lengthens" them to allow a comfortable neurulation (Fig. 31).
It is vital to preserve as generous a rim of pial cuff as possible on each edge of the placode while resecting fat along the fusion line, not just for providing sturdy stitch-holds for the micro-sutures, but a generous pial fringe also imparts a certain resilience to the neurulated placode. Very rarely, the neural placode appears on MRI to be "pressed" into a narrowbased triangle resembling a tall steep pyramid by flanking fat buttresses (Fig. 32A). The pial cuffs in such cases are also exceedingly narrow, and suturing together short pial cuffs while force-folding a squat, thick pyramid of unyielding neural tissue could result in a strangulated and ischaemic heap. The surgeon should recognize the potential hazard of the pyramidal placode on the pre-surgical MRI, and be ready to exercise judgment while reconstructing the placode (Fig. 32B-D), which may mean abandoning neurulation especially when there are adverse changes in the TcMEP during infolding and suturing of the placode edges ${ }^{55,61)}$ (Fig. 32D).
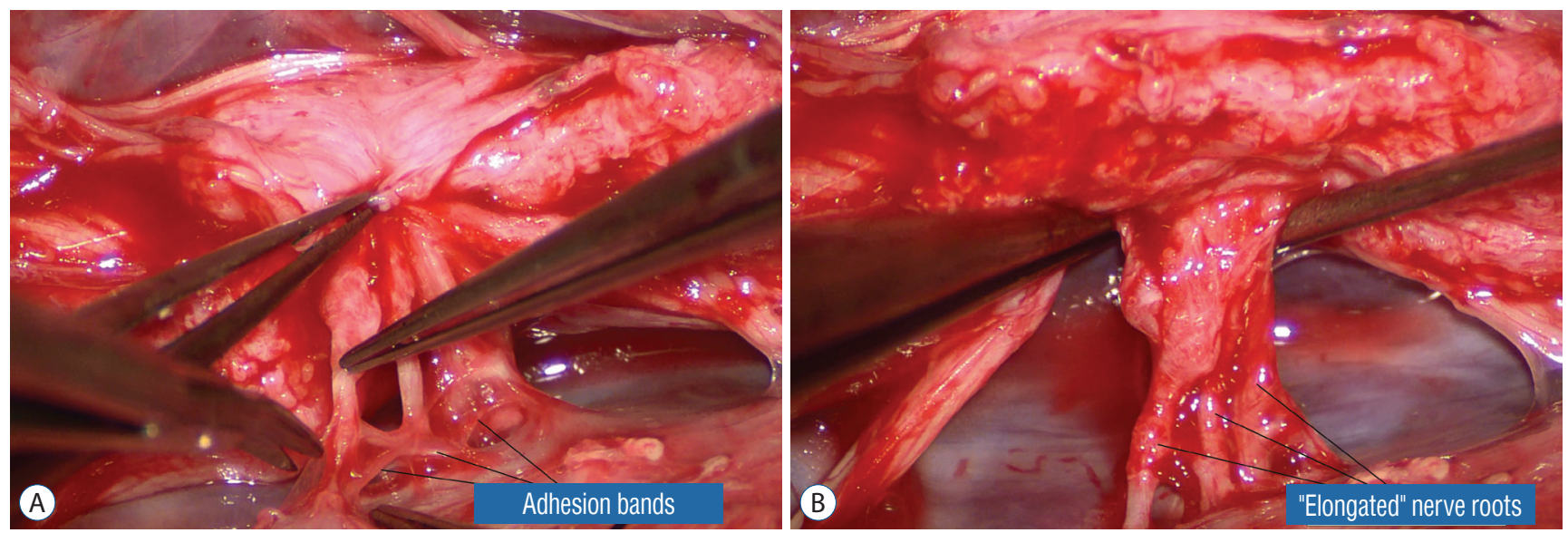

Fig. 30. Dealing with "short" nerve roots. A : The impression of shortness is spurious; these functional and supple roots appear short because they were bound tightly to the inner lining of the dura by adhesion bands that are being cut. B : After having been detached from the inner lining of the dura, these "short" nerve roots become magically "lengthened". Reprinted from Pang ${ }^{55)}$ with permission from the Japanese Neurosurgical Society.
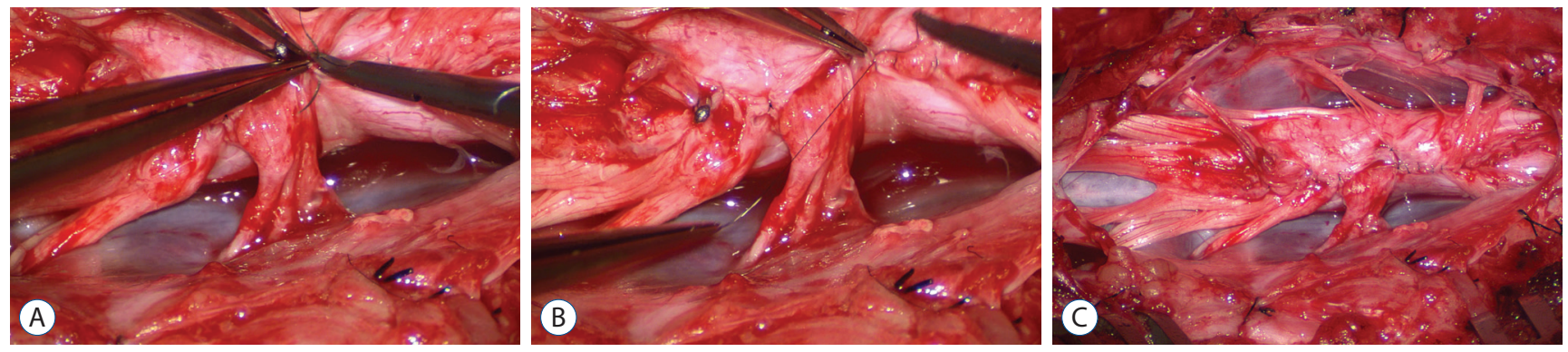

Fig. 31. Pia-to-pia neurulation of the neural placode. A : Pia-to-pia suturing with 8-0 nylon sutures with knots buried. B : Tying of micro sutures, apposing the pial fringes from each side of the placode. C: Pia-to-pia neurulation completed with interrupted 8-0 nylon micro-sutures. The reconstituted neural tube is entirely piacovered, with an unobtrusive dorsal seam. Note intact conus. Reprinted from Pang ${ }^{55}$ with permission from the Japanese Neurosurgical Society. 
If a filum is encountered after neurulation, it is usually also resected for completeness.

\section{Step 5. Expansile graft duraplasty}

Countless MRI images of loosely floating placodes and the undisputed salutary benefits of a small cord-sac ratio (see below) convinced the author that a generous dural graft ensures free movement of the placode in CSF, thereby lessening its chances of adhering to the dura. The ideal graft material must satisfy two requirements : textural compatibility with juvenile dura and a low incidence of CSF leakage through the suture holes. Gortex is too stiff for young dura and leaks CSF profusely, as does Duragen. Both materials were discarded in our early trials. Autologous fascia lata is too soft and pliant so that it collapses on to the spinal cord even during normal inspiration, and is only used when there is infection negating foreign graft materials. My preferred graft material is the full-bodied yet texturally pliable bovine pericardium (Dura-Guard; Synovis, St. Paul, MN, USA) that can maintain a "puffed-up" scaffold in all phases of respiration and postures, and shows minimal CSF leak. A close second choice is Durepair (Medtronic Neurologic Technologies, Goleta, CA USA), composed of reconstituted bovine collagen; it is slightly stiffer than bovine pericardium but does not leak. The graft is accurately shaped and sized to the dural opening to avoid creating infolds that may press on the underlying placode, which, in turn, encourages new adhesions. The edge of the graft is then carefully matched up with the dural opening and anchored with judiciously spaced interrupted $4-0$ sutures. Final water-tight closure is achieved with running 5-0 prolene sutures, followed by several challenges with Valsalva manoeuvres using pressures between 20 to $30 \mathrm{~cm}$ of water depending on the patient's size and age (Fig. 33). An inadvertent pseudomeningocoele presses the dura against the neural placode and negates any intended perquisite of capacious grafting.

\section{SURGICAL EPILOGUE : SUNDRY ADDITIONAL COMIMENTS}

The author's data show that total or near-total resection of spinal cord lipomas can be achieved in over $90 \%$ of cases $^{59-61)}$ (Fig. 34). In cases where small bits of fat were left on the DREZ for fear of gouging, they have been wrapped up within the neurulation folds "out of mischief" (Fig. 35). In $8 \%$ of patients, the residual fat represents the ventral component of a chaotic lipoma that had been intentionally left untouched and piacovered and presumably harmless.

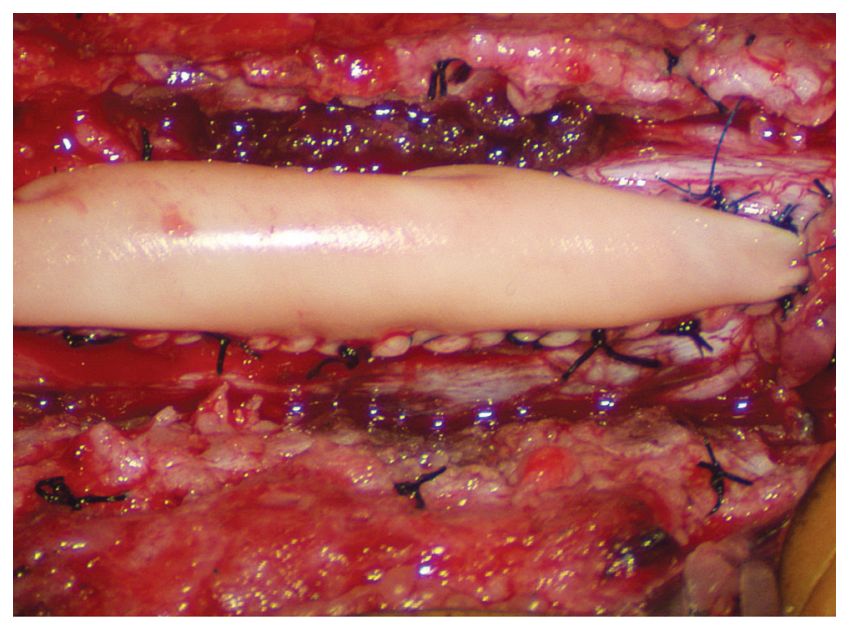

Fig. 33. Back to the dorsal lipoma in Fig. 31, after graft sutures are in place, water-tightness of the suture line is tested by several Valsalva manoeuvres. An optimal graft is one that does not leak, puffs up with expiration, and registers no inward folding, as this one. Reprinted from Pang ${ }^{55)}$ with permission from the Japanese Neurosurgical Society.
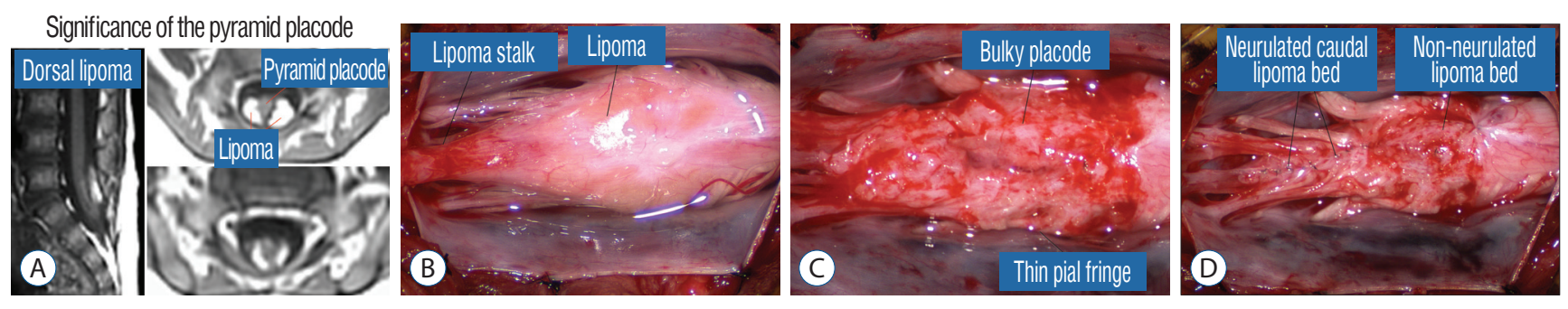

Fig. 32. Dorsal lipoma with bulky pyramid placode - lesson learned. A : Note tall pyramid-shaped neural placode on axial MRI, with steep sides slopes interfacing with the lipoma. B: Lipoma stalk and dorsal lipoma. C: After lipoma resection, the placode is bulky in the middle and has thin pial fringes. D: Only the loose caudal part of the placode was neurulated. Rostral bulky part is left open because of TcMEP deterioration on forced attempts to suture. Reprinted from Pang ${ }^{55}$ with permission from the Japanese Neurosurgical Society. MRI : magnetic resonance imaging, TcMEP : transcortical motor evoked potentials. 
To estimate the "looseness" or degree of freedom of the reconstructed placode within the expanded CSF space, we in- vented the cord-sac ratio, defined as the ratio of the diameter of the bulkiest portion of the reconstructed neural placode to
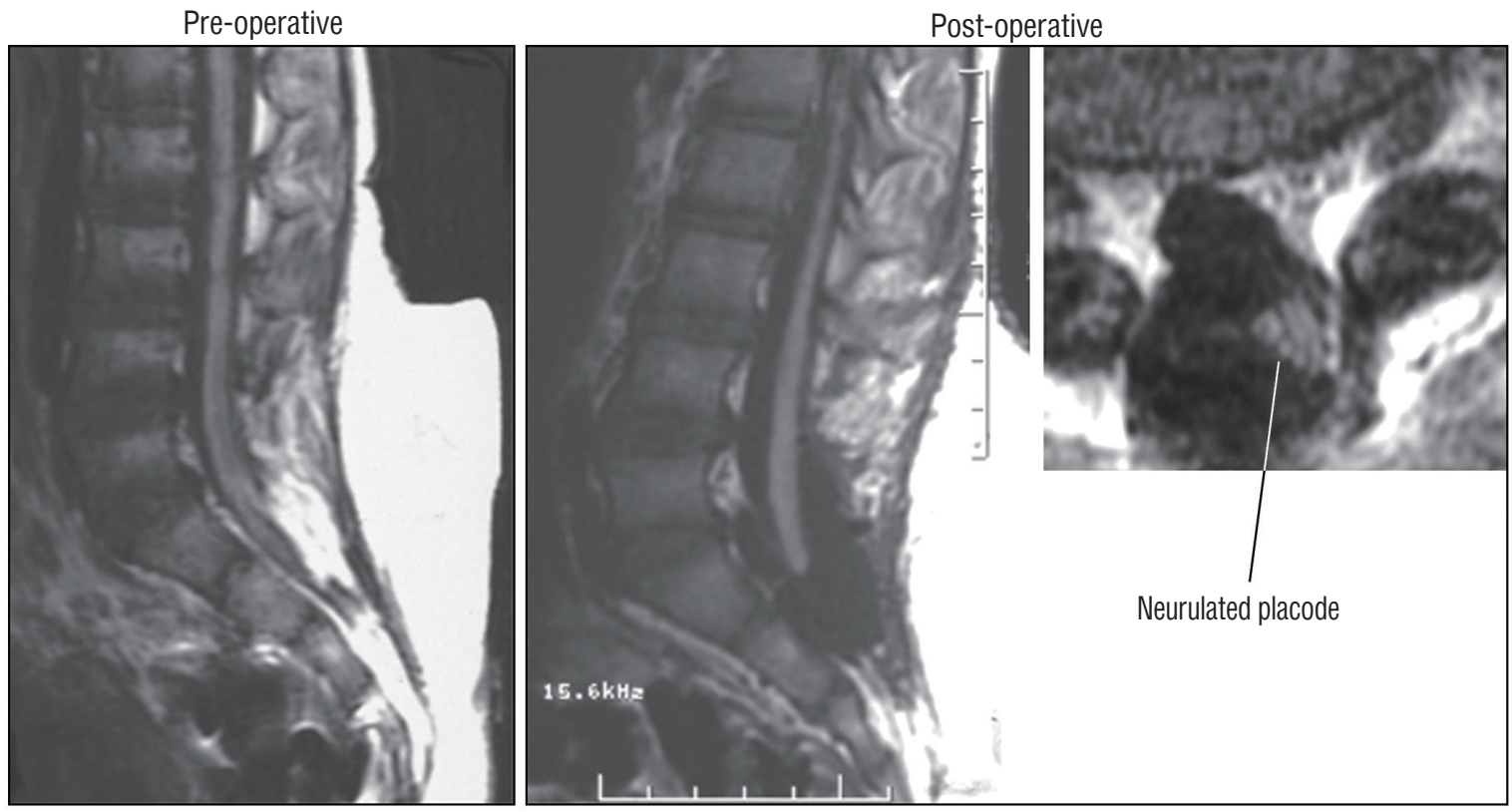

Fig. 34. Pre- and post-operative magnetic resonance imaging of a case of transitional lipoma with no residual fat after total lipoma resection. Note neurulated oblong-shaped fat-free neural placode within a large dural sac. Reprinted from Pang ${ }^{55)}$ with permission from the Japanese Neurosurgical Society.
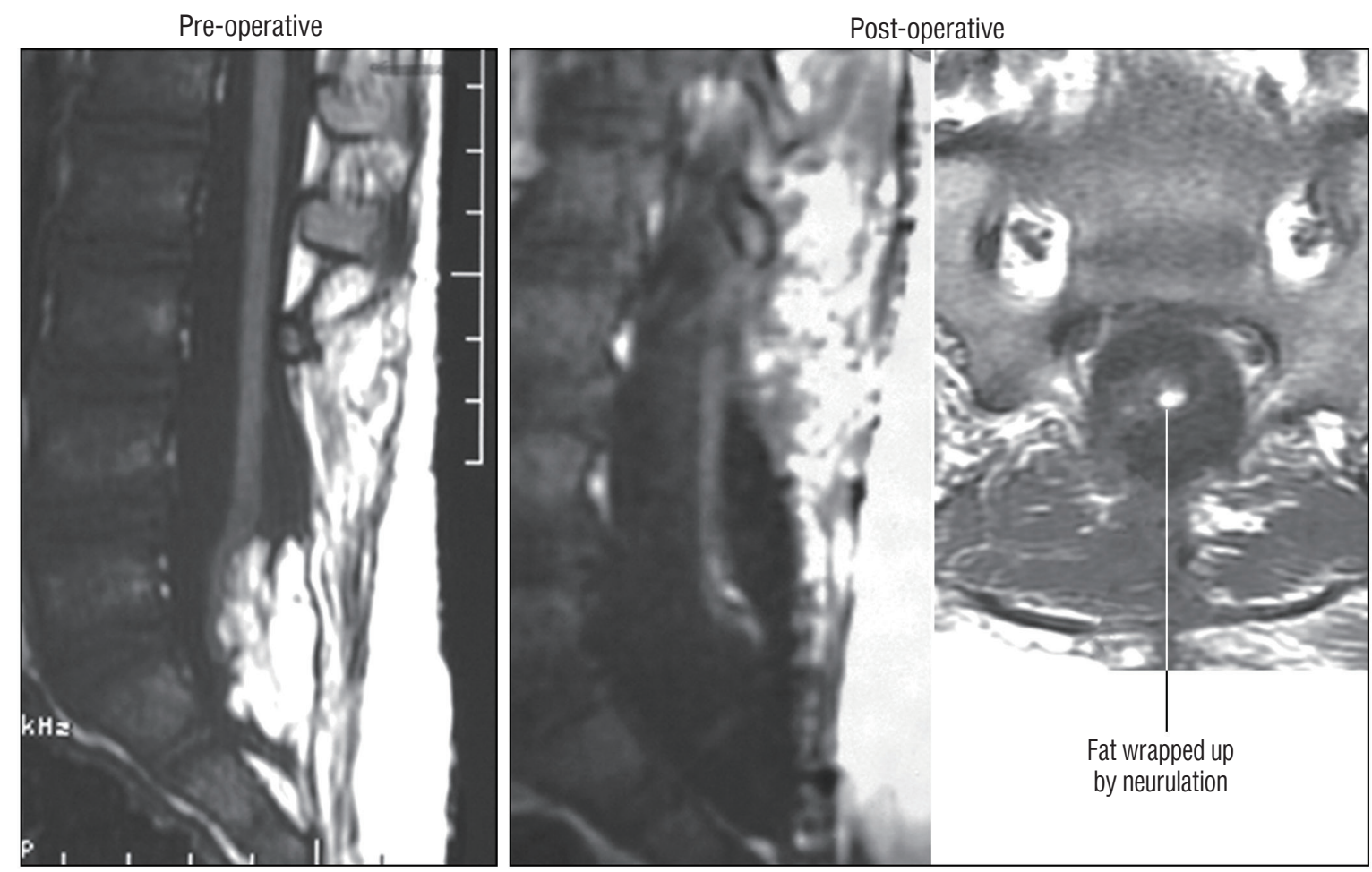

Fig. 35. Pre- and post-operative magnetic resonance imaging of a case of complex transitional lipoma with a very small amount $\left(<20 \mathrm{~mm}^{3}\right)$ of residual fat after resection. Axial image shows the small round piece of fat is wrapped up within the roundly neurulated neural placode and therefore not exposed on the surface. Reprinted from Pang ${ }^{55}$ with permission from the Japanese Neurosurgical Society. 
the diameter of the dural sac on the post-operative axial MRI. The sacs are graded as loose, with ratios less than $30 \%$; moderately loose, with ratios between $30 \%$ and 50\%; and tight, with ratios greater than $50 \%$ (Fig. 36). In our series of 315 total resections reported in 2013, 227 (72\%) had loose sacs, 73 (23.2\%) had moderately loose sacs, and only 15 (4.8\%) had comparatively tighter sacs ${ }^{61)}$.

Redo lipomas are more likely to harbour visible remainder fat and to have higher cord-sac ratios, likely because the hard, grasping fibrous scar from prior surgery cements the remainder fat to the dura and makes it extremely difficult to detach, and more importantly, the dull gray colour of the cicatrix, unlike bright yellow virgin fat, can be impossible to distinguish from the white plane. The surgeon thus tends to be over-conservative in the dissection and leaves behind a thick layer of stiff scar-infiltrated fat attached to a bulky placode that is well-nigh impossible to neurulate comfortably. The same unyielding fibrous muddle at the DREZ often leads to "gouging", which may explain the high incidence of post-operative dysesthetic pain in "redo" versus "virgin" patients".

Incomplete terminal untethering of the placode predictably ends in early clinical relapse ${ }^{9,62,74)}$. In exceptionally large transitional lipomas, the caudal placode is mired in fat, and its com- plete detachment cannot be done safely without substantial removal of fat. Also, healthy looking nerves are sometimes seen enmeshed in fat distal to what was reckoned to be the tip of the placode, making it seem impossible to achieve terminal disconnection without sacrificing functional cord. Electrical stimulation of these nerves will find them to be non-functional vestigial coccygeal roots. In such cases, 2 to 3 sets of "live" anal sphincter roots should be located on the conus proper above, so that a decisive transection of the vestigial placode below will achieve complete terminal untethering without sacrificing function ${ }^{58-61)}$.

Our experience unequivocally shows that chaotic lipomas are the most treacherous lesions. Some chaotic lesions are easily recognisable by the presence of obvious ventral fat in between pairs of ventral roots on the MRI, but other less blatant ones have sub-resolution trails of fatty infiltration through some of the DREZ into the ventral motor horns zone, detectable only at surgery (Fig. 26A). Compared to other lipoma types, chaotic lipomas are more likely to show conspicuous residual fat and high cord-sac ratio on the post-operative $\mathrm{MRI}^{59-61)}$ (Fig. 26D). The strategy for these difficult lipomas is learning to resist digging too deep once enough dorsal fat has been removed to enable partial neurulation on the dorsal por-
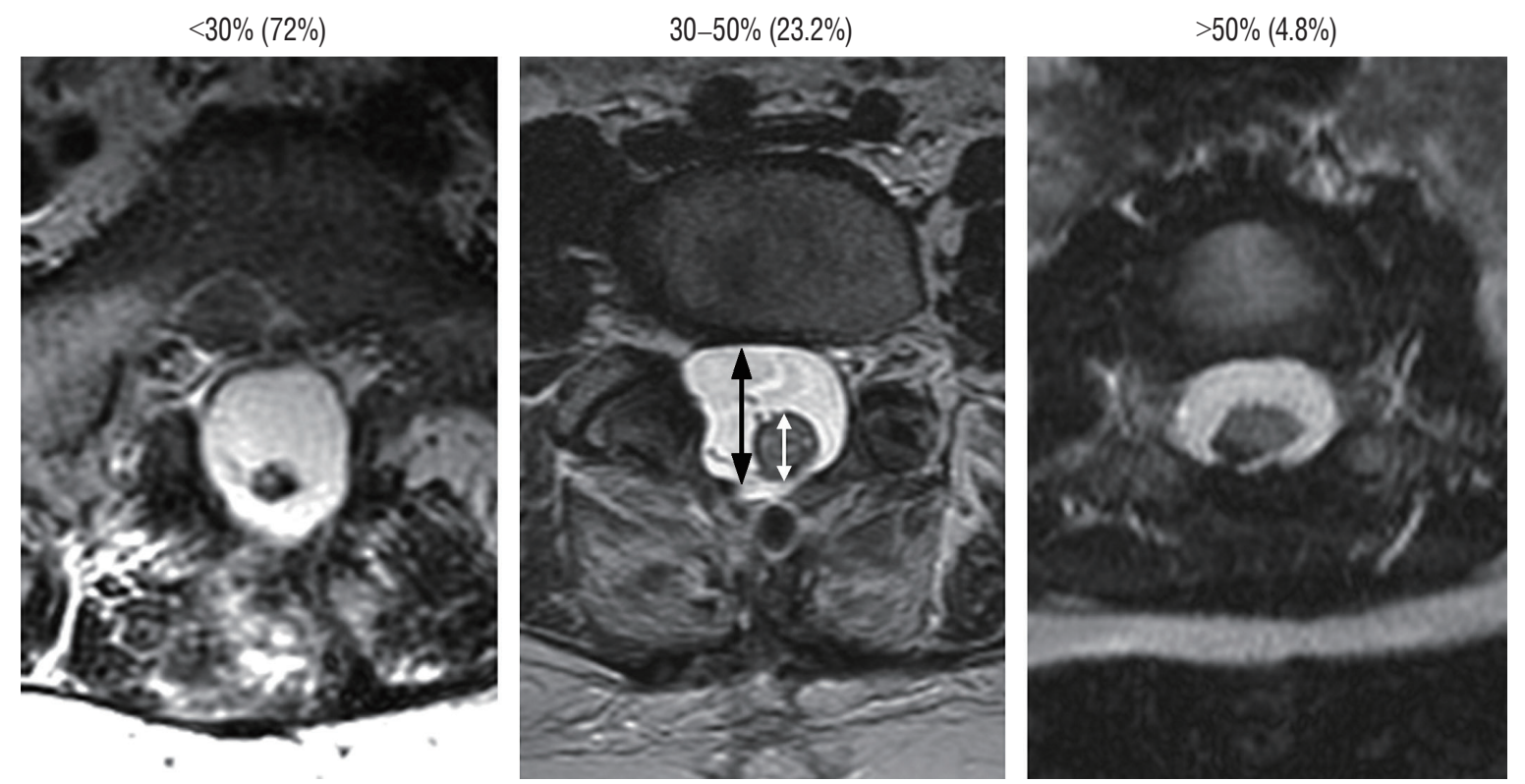

Fig. 36. Cord-sac ratios in the post-operative axial magnetic resonance imaging after total/near-total resection of lipoma. This ratio is obtained by dividing the sagittal diameter of the most bulbous portion of the post-neurulated neural placode (white arrow) by the sagittal diameter of the dural sac (black arrow). $72 \%$ in our series have very loose sacs with cord-sac ratios $<30 \% ; 23.2 \%$ have intermediate ratios $30-50 \%$; and $4.8 \%$ have ratios $>50 \%$ with the least commodious cordsac relationship. Cord-sac ratio estimates the degree of freedom of motion of the post-neurulated spinal cord within its container sac. Reprinted from Pang ${ }^{55)}$ with permission from the Japanese Neurosurgical Society. 
tion of the lipoma. Since it is never feasible to totally resect the ventral fat or to achieve even clumsy neurulation, its nascent pial surface that had presumably existed without adhesions since birth, should best be kept unabraded so as not to incur iatrogenic tethering (Fig. 26C).

Finally, I disagree with Arai et al. ${ }^{1)}$, Chapman ${ }^{8}$, Chapman and Davis", and Kulkarni et al. ${ }^{27)}$ that "lipomyelomeningocoeles", or what I call lipoma with extraspinal extension, have higher surgical risk and worse outcome than "regular lipomas". As long as one recognises and gently handles the extruded part of the neural placode beyond the dorsal plane of the spinal canal, the technical steps of fat resection, placode reconstruction, and dural grafting are identical to those used for the more orthodox lipomas, with comparable results. I also think that Kulkarni et al. ${ }^{27)}$, and Chapman's ${ }^{8)}$ warnings about lipomas in adults being technically more challenging are unwarranted. Except perhaps for thicker "natural" arachnoid bands in adults and more laborious bone and fascial exposure, most of the microsurgical work is the same as for resecting lipomas on young children and infants.

\section{COMPLICATIONS OF TOTAL LIPOMA RESEC- TION}

Our rate of new neurological and urological symptoms following total/near-total resection is $4.1 \%{ }^{61)}$, which compares favourably with the $0.6 \%$ to $10 \%$ (average of 3-7\%) in the literature $^{1,2,10,19-21,25,26,29,37,38,41,63,64,70,73)}$, all pertaining to partial resection presumably using less aggressive techniques than ours. Only $1.5 \%$ of our patients had new weakness but $4 \%$ had neuropathic pain ${ }^{59-61)}$, which could be related to mechanical or thermal perturbation near the DREZ from diathermy and gouging. This has taught us to avoid cautery as much as possible and use gentle tamponade and gelfoam. If diathermy is absolutely necessary, we use only the ultra-fine tip irrigating bipolar set at the lowest current intensities, always precisely localizing the bleeding spot using the intermittent squirt-irrigation technique from the bubble-squeezer.

Our rates for CSF leak $(0.7 \%)$ and wound complications (1.1\%) with total resection ${ }^{61)}$ are much lower than almost all of the published series (of partial resection), which report CSF leak rates from $2 \%$ to $47 \%{ }^{10,19,25,26,29,41,63,70,74)}$ and wound dehiscence and infection rates of $2 \%$ to $26 \%{ }^{19,22,25,29,41,63,70,74)}$. The fol- lowing time-tested technical points concerning wound closure are worth noting : 1) adequate caudal laminectomy is necessary to locate a healthy dural edge at least $5-10 \mathrm{~mm}$ past the tip of the neural placode to sustain good suture hold for the lower end of the graft anastomosis, which is always the tenuous end. Ideally, this very edge should be sturdy dura and not fat of the distal lipoma stump. A good caudal dural edge is usually available with dorsal lipoma whose fatty stalk ends rostral to the conus as does the dural defect, but in some very large transitional lipomas, the entire conus is involved in the lipoma and the caudal dural edge can be partly or wholly infiltrated by fibrous fat. The surgeon will have to improvise on finding the best possible suture hold for the lower end of the graft, occasionally resolving to using "big bites" on fibrous fat or through flakes of the incomplete deep fascial layers. Fortunately, this is an uncommon situation. 2) Patience and ingenuity must be used to achieve absolute water-tight closure of the graft with Prolene, followed by Valsalva manoeuvres. 3) We are not as enchanted by synthetic sealants or organic tissue glues for the graft anastomosis as before, for nothing can substitute for solid closure. Use of these agents is optional in cases of tenuous suture line. 4) In large lumbosacral lipomas, gaping muscle and fascial defects may forestall primary approximation of the deep layers at the lower end of the opening. An effective solution is to create generous paramedian relaxing incisions on the flanking lumbodorsal fascia to allow the fascial edges to slide towards the midline and be primarily closed without tension. And 5) the large subcutaneous lipoma is never removed during the initial soft tissue dissection to avoid creating a large dead space that could potentially encourage CSF leakage and formation of a tense pseudomeningocoele. The latter may compress and flatten the dural sac against the placode or even threaten the viability of the skin flap.

\section{RESULTS OF TOTAL RESECTION}

The results of total resection is best analysed by comparing its outcome with that of the traditional technique of partial resection. This has proven to be a convenient enterprise for the author, who had performed 116 partial resections prior to 1991, using the same meticulous adherence to technical details as for the total resection series. These comparisons are done in two parts, that of the immediate post-operative results and the 
long term outcomes of these two different techniques.

\section{EARLY POST-OPERATIVE RESULTS}

The immediate effects of surgery during the first 12 months are very similar between our own total and partial resection groups $^{59-61)}$. For asymptomatic patients, the rates for neurological preservation are $98 \%$ for total and $94 \%$, for partial resection, respectively. For symptomatic patients who had total resection, $61 \%$ had normalisation or improvement, and 33\% had stabilisation of neurological status, giving a combined rate of $94 \%$ for improvement or stabilisation of disease. Similarly, symptomatic patients who had partial resection had a $33 \%$ improvement and $62 \%$ stabilization rates, thus also having a $95 \%$ rate of short term protection.

When the early post-operative results are compared between our total resection group and partial resection series from the literature ${ }^{1,10,19,29,41,64,74)}$, it is again apparent that both the disease improvement and stabilization rates are very similar, particularly among patients with progressive symptoms. This suggests that the immediate benefits of surgery in the early post-operative period, for both techniques, are owed to the abrupt interruption of the tethering effect on the cord rather than the amount of lipoma removal or work on the placode. In contrast, surgeons who lamented their inability to completely detach the terminal placode from the lipoma stump in some patients, due to massive caudal fat and undefinable placode-lipoma anatomy, also reported their early recurrence of symptoms ${ }^{9,63,74)}$.

When the catalogue of pre-operative symptoms are analysed in regards to their response to total resection, the abatement of pain came out the best and most reliable. Typically, the sharp, dysesthetic pain in the lower limbs and perineum will significantly diminish within 3 months ${ }^{61}$. Relief of low back pain, which may be mechanical in origin, is less reliable ${ }^{18,57)}$. Children do not usually complain of back pain but they do seem to become more active and playful. Active sensory symptoms such as dysesthesia and hyperpathia also respond favorably with surgery. Although only $20 \%$ of patients have actual normalisation of motor deficits, the majority with pre-operative weakness will substantially improve ${ }^{59-61)}$. Like tethered cord syndrome in general, recent and milder deficits and symptoms have better chance for good recovery than severe chronic ones. Unlike pain and sensorimotor deficits, bladder dysfunction rarely improves significantly and if so, the recovery is never complete, and partial recovery occurs in no more than $20-30 \%$ of patients ${ }^{59,61)}$. Amongst the several subtypes of neuropathic bladder, the spastic, small capacity bladder with uninhibited detrusor contractions tends to respond best to surgery. The atonic bladder virtually never improves to a point of not needing permanent intermittent catheterization, and the response of detrusorsphincter dyssynergia is at best partial. It is strongly advisable to obtain formal cystometry and voiding cystourethrogram 3-6 months after surgery and yearly thereafter if indicated to assess whether more radical urologic procedures such as bladder augmentation or ureteral conduits might be needed to eliminate reflux and upper urinary tract infections.

\section{LONG-TERM OUTCOME}

Whilst the immediate benefits of both total and partial resection are due to the equivalent relief of traction on the conus in both procedures and are therefore comparable ${ }^{59-61)}$, the long term benefits of total over partial surgery probably come from the sustainment of the untethered state when the lipomatous cord underwent aggressive fat removal and protective reconfiguring of the neural structure, an undertaking obviously

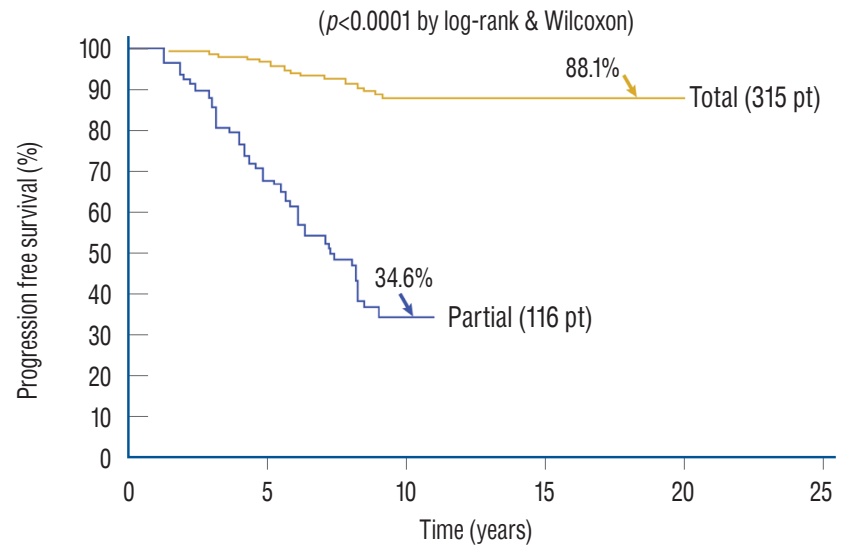

Fig. 37. Outcomes of total versus partial resection. Kaplan-Meier (KM) analysis for progression-free survival probability in total and partial resection of lipoma. The progression-free probability for total resection is $88.1 \%$ at 20 years, and $34.6 \%$ for partial resection at 10.5 years. The difference is highly significant ( $p<0.0001$ by log-rank \& Wilcoxon). Note stabilization of disease after 8 years with total resection, but inexorable deterioration without disease arrest with partial resection. Reprinted from Pang ${ }^{55)}$ with permission from the Japanese Neurosurgical Society. $\mathrm{Pt}$ : patients, Total : total resection, Partial : partial resection. 
unobtainable with partial resection. This is made very plain when the progression-free survivals (PFSs) of our own series of total and partial resection are plotted out for a period of 20 and 11 years, respectively. The PFS after total resection for all lipoma types and clinical subgroups is $88.1 \%$, versus $34.6 \%$ for partial resection ${ }^{61)}$ (Fig. 37). The differences in outcome between the two techniques are even more marked if the subgroup PFSs are individually compared, when the patients in each treatment group are segregated by the presence or absence of pre-operative symptoms, age, and whether previous surgery had been done ${ }^{59-61)}$. Overall, the risk of symptomatic recurrence is 5.94 times higher with partial resection compared with total/near-total resection over 18 years by the Cox Proportional Hazard Regression Model, with a $p$-value $<0.00001$. This striking difference in outcomes between total and partial resection is equally obvious when the results of our total resection group are held up against the published results of partial resection ${ }^{1,8,11,12,41,63,64,67,68,70,74)}$, especially if the comparisons are done with matched patient profiles (most published series include only virgin cases whereas ours has

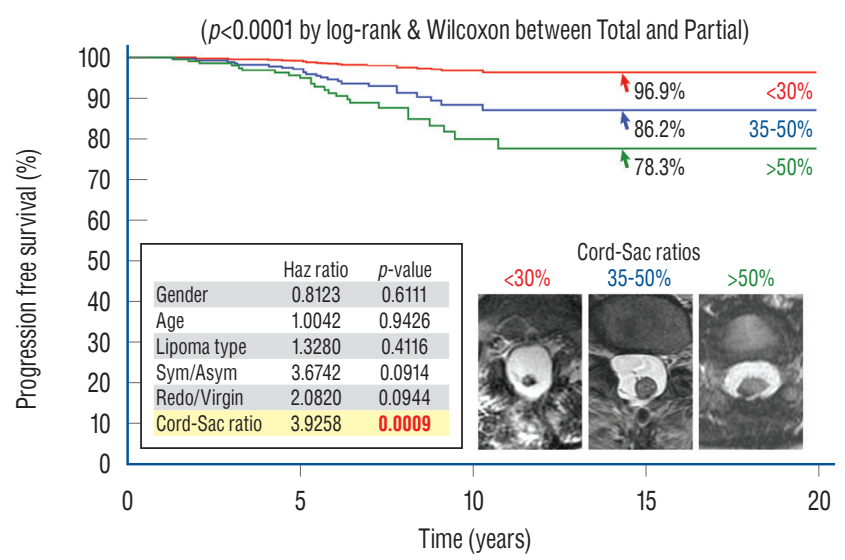

Fig. 38. Cox Multivariate Proportional Hazard Regression model analyzing the combined influence of six predictor variables (gender; age; lipoma type; symptoms; redo versus virgin; and cord-sac ratio) on PFS after total resection, featuring the resultant effect of the three cord-sac ratios of $<30 \%, 30-50 \%$, and $>50 \%$. The hazard ratios and $p$ values for all six predictor variables are listed in the miniaturized table, showing that cord-sac ratio exerts the only significant independent influence on outcome. The respective progression-free probabilities, as indicated by the arrows, are $96.9 \%$ for low ratio, $86.2 \%$ for intermediate ratio, and $78.3 \%$ for high ratio. The differences in hazard prediction for the three ratios are highly significant ( $p=0.0009$ in bold). $<30 \% ; 30-50 \%$, and $>50 \%$ indicate the three cord-sac ratios as shown in magnetic resonance imaging insets. Reprinted from Pang ${ }^{55)}$ with permission from the Japanese Neurosurgical Society. Sym/Asym : symptomatic versus asymptomatic lipomas. both virgins and redos) and lesion types (many published series include terminal and filar lipomas which are technically far less complicated and have better prognosis). Barring these and other minor criticisms (e.g., our data are prospective whereas most published series are retrospective), these elaborate comparisons all show far better long term prognosis with total/near total resection, which constitutes the best reason for its endorsement over all other treatment options for complex spinal cord lipomas.

\section{Predictor variables influencing outcome}

In 2010, we attempted to find the actual reason(s) why total resection gave such a stunning advantage over partial surgery, by investigating the influence of six predictor variables on PFS in the series ${ }^{60)}$. These variables are gender, age, lipoma type (dorsal, transitional and chaotic), presence or absence of preoperative symptoms, history of previous resection, and cordsac ratio. Simple Cox univariate analyses show that gender and lipoma type have no influence on outcome, but that young age, absence of symptoms, no prior surgery, and a low cord sac ratio are all associated with statistically longer PFSs. However, when age, symptoms, and prior surgery (not cordsac ratio because it is a post-operative entity) are tested with the Cox multivariate model with all six variables inserted in the analysis, the significant $p$-values associated with their univariate PFS curves all disappeared, suggesting the 3 are in fact

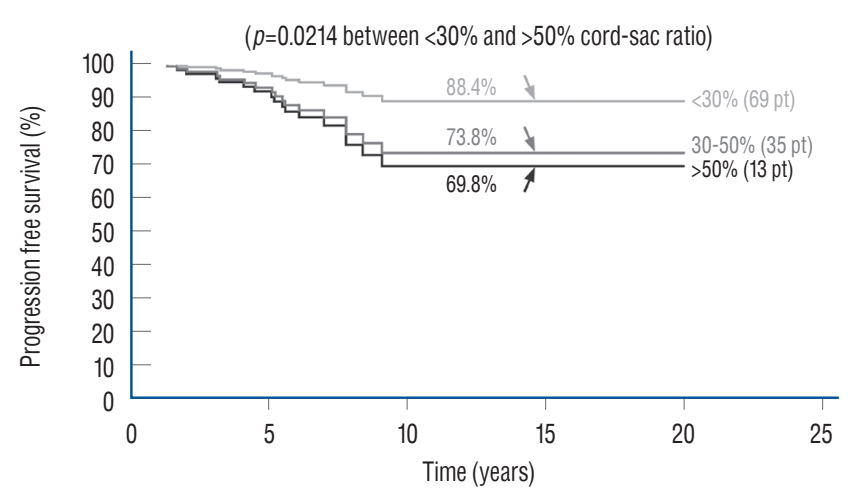

Fig. 39. Cox Multivariate analysis for the influence of cord-sac ratios on outcome in 117 redo lipomas that underwent total resection. Progressionfree probabilities are indicated by arrows. A high progression-free survival of $88.4 \%$ can still be managed even in redo lipomas if a cord-sac ratio $<30 \%$ can be achieved, indicating the dominant role of cord-sac ratio, and conversely the subordinate role of other unidentified attributes of previous surgery, on long-term outcome $(p=0.0214$ between $<30 \%$ and $>50 \%$ cord-sac ratios). Reprinted from Pang et al. ${ }^{61)}$ with permission from Springer Nature. Pt : patients. 
"non-independent" predictors of outcome ${ }^{60)}$. The same statistical exercise was performed in our 2013 series of 315 patients, with similar results ${ }^{61)}$. The inevitable conclusion is that these three predictor variables must exert their influence on outcome through some sort of final common pathway ${ }^{59-61)}$.

Unlike the three non-independent predictor variables of age, symptoms and prior surgery, cord-sac ratio emerges as the solitary independent predictor of outcome shown clearly by its highly significant influence on progression-free probabilities displayed by the Cox multivariate model ${ }^{61)}$ (Fig. 38). It is therefore tempting to suggest that cord-sac ratio may in fact be the "final common pathway" for the three non-independent predictors. To test this hypothesis on the influence of prior surgery on outcome, we examine whether redo lipomas' negative effects on PFS can be explained solely by their having high cord-sac ratios. The first positive clue is that redo lipomas tend to end up with much higher post-operative cord-sac ratios than virgin lipomas ${ }^{59-61)}$. Secondly, analysis of covariance (ANCOVA) clearly portrays the same strong correlation between prior surgery and high cord-sac ratios $\left(\mathrm{R}^{2}\right.$ correlative coefficient, $0.76 ; p<0.001)^{61)}$. Last and most revealing, when the Cox multivariate regression analysis is performed on cordsac ratio for just the redo lipomas, a PFS of $88.4 \%$ is still manageable in redo lesions as long as a less than $30 \%$ cord-sac ratio can be rendered ${ }^{61)}$ (Fig. 39). This proves that redo lesions can still enjoy good outcome as long as a low cord-sac ratio can be achieved, and clearly shows the subordinate role of other un-

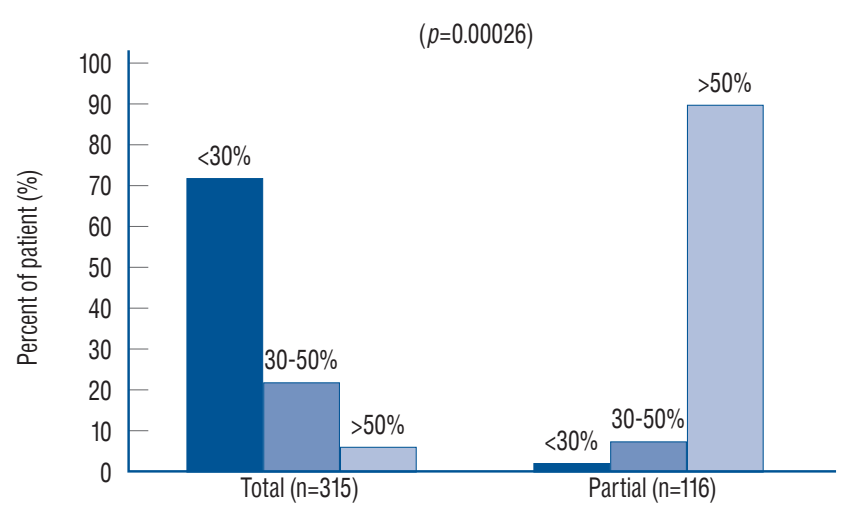

Fig. 40. Distribution of cord-sac ratios between the total resection and partial resection groups. Note $72 \%$ of patients who had total resection had cord-sac ratio $<30 \%$, versus less than $3 \%$ of patients who had partial resection. Conversely, only $6 \%$ of patients who had total resection had high ratio of $>50 \%$, versus over $90 \%$ of patients who had partial resection. The difference is significant $(p=0.00026) .<30 \% ; 30-50 \%$; and $>50 \%$ indicate the cord-sac ratios. Reprinted from Pang ${ }^{55)}$ with permission from the Japanese Neurosurgical Society. identified attributes of prior surgery. This same train of analyses, including the respective covariance analysis, can be applied to the other two "non-independent" predictors, age and symptoms, with similar conclusions.

\section{Cord-Sac ratio and the importance of neurulation}

My own records show a far higher likelihood for total resection to achieve low cord-sac ratios than partial resection, being $72 \%$ in the total group versus only $3 \%$ in the partial group (Fig. 40), suggesting that cord-sac ratio is probably what determines the difference in outcome between the two techniques $^{61)}$. For the total resection group, Cox Multivariate analysis of the combined influence of the 6 predictor covariates shows that cord-sac ratio's highly significant, independent influence on outcome is unchanged by howsoever the other effects of age, symptoms, lipoma types, and prior surgery interact (Fig. 38). Regardless of all else, a cord-sac ratio greater than $50 \%$ predicts a 5.3 times higher risk for disease progression than a low cord-sac ratio of less than $30 \%$, with a high statistical significance (Figs. 38 and 41). The PFS rises to a reassuring $96.9 \%$ for low ratios less than $30 \%$, and drops gradually to $86.2 \%$ for intermediate ratios between $30 \%$ and $50 \%$, and $78.3 \%$ for ratios higher than $50 \%{ }^{61)}$.

In an operational sense, cord-sac ratio may in fact be the summated product of the other group variables. For example,
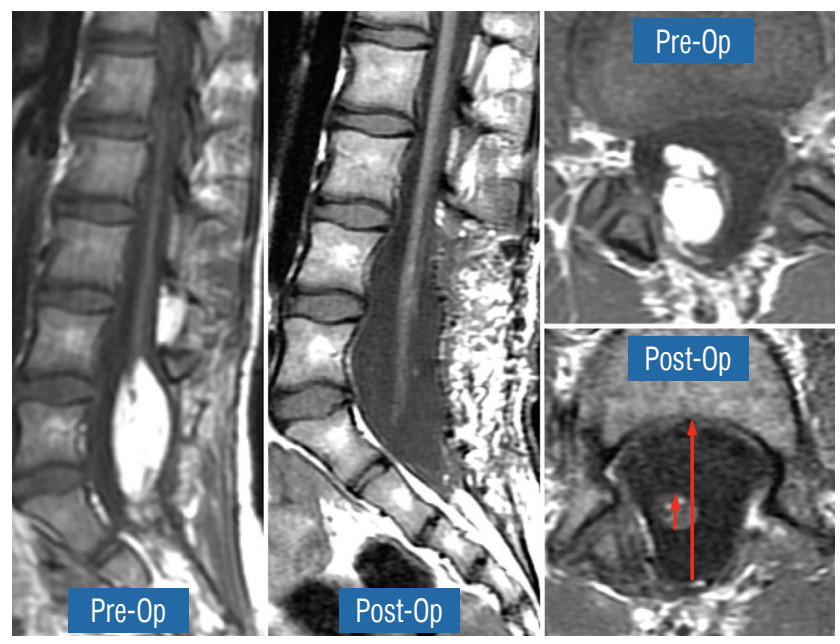

Fig. 41. The pre- and post-operative sagittal and axial magnetic resonance imaging of a 10 years old girl who has had two previous partial resections of a large transitional lipoma, and who developed new leg weakness. The achieved post-operative cord-sac ratio is $20 \%$ (lower right); and she enjoyed long term progression-free status. Reprinted from Pang ${ }^{55)}$ with permission from the Japanese Neurosurgical Society. 
previous surgery imparts new scar tissue on the remainder fat, whose altered hue and texture undoubtedly makes it near impossible to separate lipoma from white plane and therefore to attain a clean, slender placode and a small cord-sac ratio. Since many symptomatic patients in our total resection group have had prior partial resection, their poorer outcome may be largely due to the negative influence of the "redo factor", which may also account for the disadvantage of old age since most elderly patients had both symptoms and prior surgery before their total resection.

Though a loose fitting sac ensures a greater degree of free movements of the placode within CSF, a condition known to discourage retethering, adhesions can still form if what is facing the dura is a broad, sticky, raw resection surface on a sheetlike placode. Meticulous pia-to-pia suturing conceals this sticky surface within a smooth pia-covered tube and further lessens the chances of adhesion. Reexploration of both neurulated and unneurulated placodes shows unequivocally that the raw unneurulated slab is much more of a conspicuous nidus

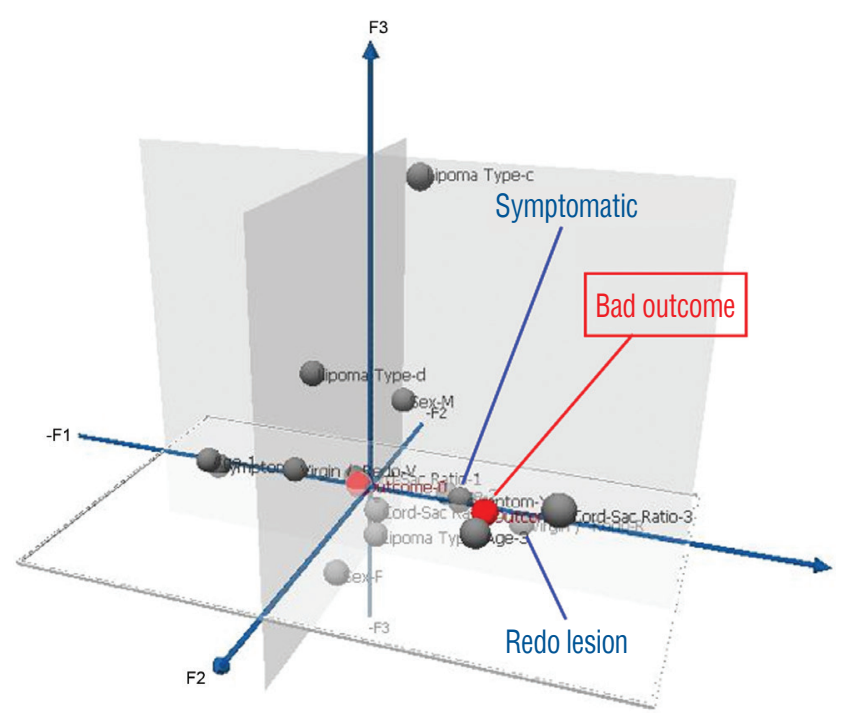

Fig. 42. Pre-operative profiling of good versus poor risk patients for total resection using three dimensional Multiple Correspondence Analysis plot, which displays the respective strength of influence of the six predictor variables (gender, age, lipoma type, symptoms, redo versus virgin lipomas, and cord-sac ratio) on outcome after total resection. The grey balls represent predictor variables and red balls represent outcomes. Only the statistically significant predictors are flagged. Bad outcome implies recurrence and good outcome the absence of recurrence during the follow-up period. Close clustering of variables (with flags) around an Outcome signifies strong influence; remote scattering of variables (without flags) from an outcome signifies weak influence. Bad outcome is associated with pre-operative symptoms and redo lesions. Reprinted from Pang et al. ${ }^{611}$ with permission from Springer Nature. for new adhesions than a smooth tube with a discrete seam. Concealment of this adhesive surface through minutely careful neurulation must be at least as essential and indispensable as a low cord-sac ratio in guarding against retethering.

\section{Pre-operative profiling for good and poor risk patients}

In clinical practice, it is important to inform patients and families of their chances of success before the recommended treatment, and for that, the post-operative index of cord-sac ratio, though by far the most potent outcome predictor, will not be available. To create a pre-surgery composite patient profile to differentiate potentially favourable from unfavourable surgical candidates, the strengths of influence on outcome of all pre-operative traits derived from the predictor variables are ranked on a graded map from another multivariate data analysis model, Multiple Correspondence Analyses (MCA). In an MCA 3-dimensional map, the individual strength of influence on outcome of each of the 5 pre-operative predictor variables of age, gender, lipoma type, symptoms, and prior surgery is graded so that its magnitude is inversely proportional to its distance from a pertinent outcome category, displayed on a "principal component axis" on the map. A short distance from the outcome category indicates strong influence, and conversely, a long distance indicates minimal influence. Thus, at a glance, the traits clustering closest to the

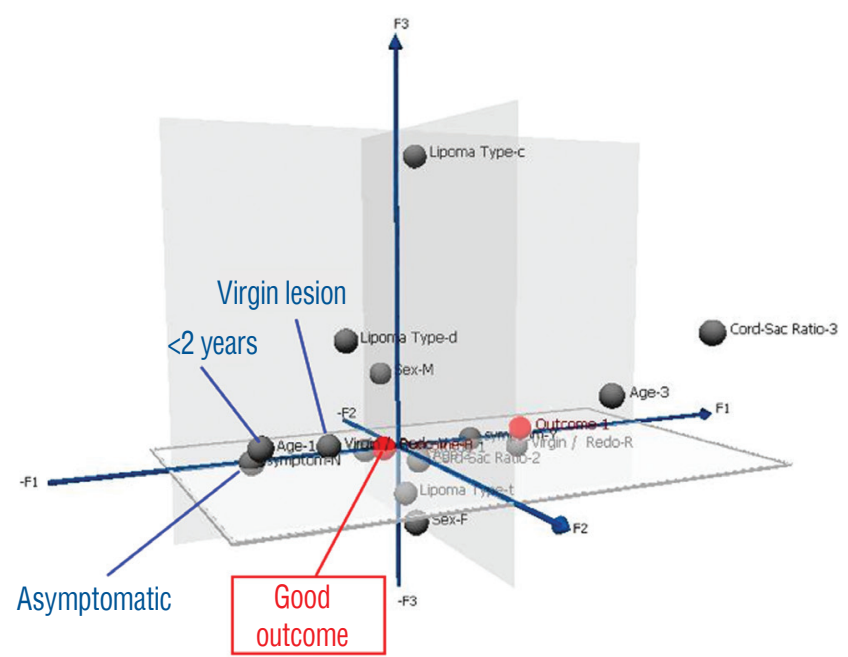

Fig. 43. Same pre-operative profiling as in Fig. 42 for good outcome, using the same Multiple Correspondence Analysis plot. Good outcome is associated with children less than 2 years, asymptomatic lesions, and virgin lipomas. Reprinted from Pang et al. ${ }^{61)}$ with permission from Springer Nature. 
outcome category on the MCA map would have dominant influence and, vice versa, those traits far afield would have little or no influence. Outcome is considered "bad" for those who had post-operative recurrence of symptoms, and "good" if no recurrence is documented during the follow-up period ${ }^{59-61)}$.

The MCA map shows that bad outcome is strongly associated with lipomas with symptoms and prior resection (Fig. 42). Good outcome is, not unexpectedly, correlated with young age especially less than 2 years, absence of symptoms, and no prior surgery (Fig. 43). Capitalising on the MCA results, we performed Cox regression on various patient groups that had total resection and found that the PFS probability of the 84 patients that fit this ideal profile, i.e., asymptomatic children younger than 2 with no previous resection, maintains at a stunning $99.2 \%$ at 20 years (Fig. 44). The ideal scenario with the highest probability of obtaining long term disease control is therefore to perform total resection on very young children before symptoms arise, and most definitely before partial resection has been inflicted. Indeed, our subgroup of 139 patients who had their virgin lipomas resected when young all had post-operative cord-sac ratios $<30 \%$, and none had recurrence during the 20 years follow-up period.

In stark contrast, older patients with pre-operative neurological dysfunctions and prior surgery will have a much less sanguine future.

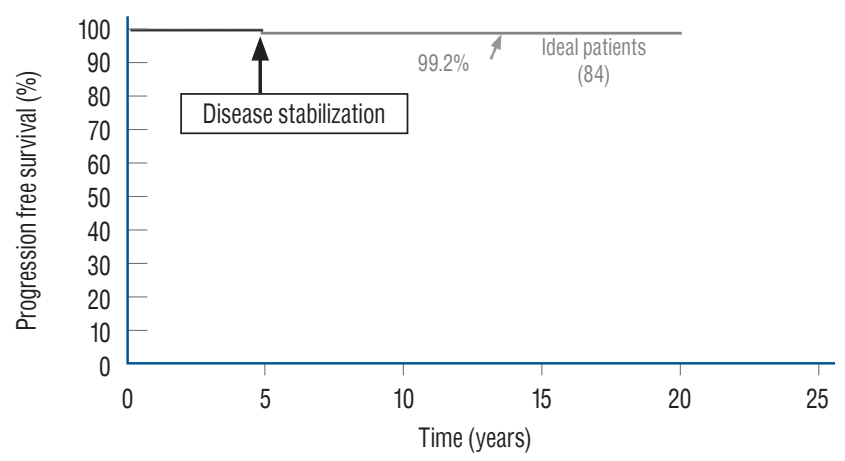

Fig. 44. Kaplan-Meier analysis for progression-free survival showing the predicted outcome of the 84 "ideal patients" who had had total resection; i.e., asymptomatic children younger than 2 years with virgin lipomas. The progression-free probability at 20 years is $99.2 \%$, with disease stabilization after 5 years from surgery. Reprinted from Pang et al. ${ }^{61)}$ with permission from Springer Nature.

\section{TOTAL RESECTION VERSUS NO SURGERY FOR ASYMPTOMATIC LIPOMAS}

Above all, the most important and certainly most often asked question is undoubtedly whether surgery should be offered to children with asymptomatic lipomas. In 2004, a cohort of 50 children with asymptomatic lipomas were prospectively followed without surgery for 9 years in Paris ${ }^{27)}$, and were found to have a deterioration risk of $33 \%$ with a PFS probability of $67 \%{ }^{27)}$. A retrospective study from London, with similar patient numbers (56 children) and profiles also reported a 10 years disease progression rate of $40 \%$ and PFS of only $60 \%$ with non-surgical treatment ${ }^{73)}$. The London study also found that the female gender, transitional lipomas, and conus syringes are all associated with a higher rate of deterioration which also occurs earlier than patients not having these traits. Since transitional lesions and females are usually more numerous in most lipoma cohorts, as are conus syringes, one could surmise that there is a large subclass of children with these traits who will have a worse than $40 \%$ prospect of disease progression $^{48)}$ (Fig. 45). Given that children within the lipoma age range have an actuarial life span of 65 to 70 years, and that established neurogenic bladder and motor weakness seldom fully recover following post factum salvage surgery $^{2,6,29,41,70,74)}$, the push for preemptive surgery for asymptom-

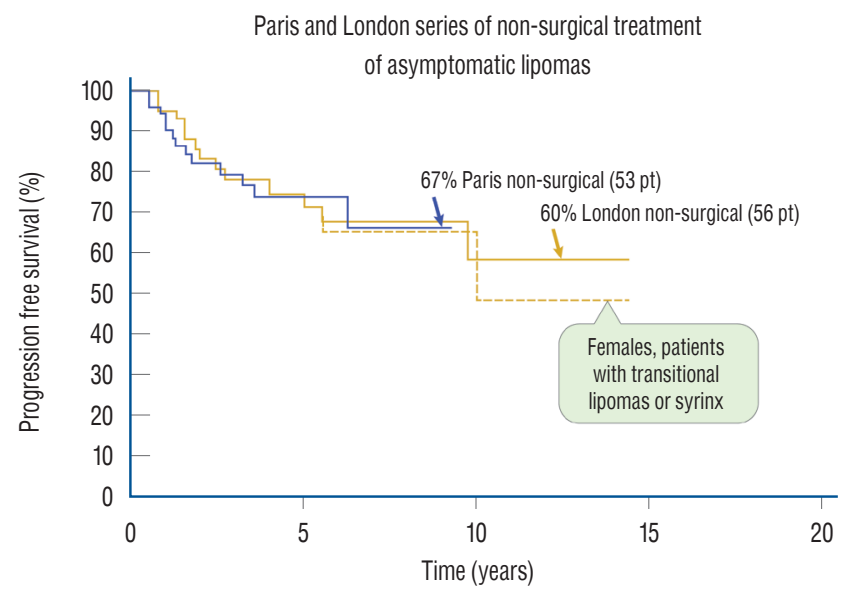

Fig. 45. Non-surgical treatment of asymptomatic lipomas. The blue line denotes the Paris series of 53 patients followed prospectively for 9 years ${ }^{66)}$, with a PFS of $67 \%$. The yellow line denotes the retrospective London series of 56 patients followed for 10 years ${ }^{68)}$, with a PFS of $60 \%$. In the London series, females, those with transitional lipomas and conus syrinx did worse, thus with an even worse PFS (dotted yellow line). Reprinted from Pang ${ }^{55}$ with permission from the Japanese Neurosurgical Society. Pt : patients, PFS : progression-free survival. 
atic lipomas would seem logical if it can be shown to offer clear-cut outcome advantage over non-surgical management.

Total resection, as is amply shown here, does just that. When the subgroup of 113 asymptomatic children with virgin lipoma from our total resection cohort is put up against the Paris and London groups, the superiority of total resection is obvious, with a 20 years PFS of $98.8 \%{ }^{61)}$ compared to the 10 years rates of $67 \%$ and $60 \%$ for conservative management in Paris and London, respectively ${ }^{27,72)}$ (Fig. 46). In addition, our total resection PFS curve shows disease stabilisation 5 years after surgery, whereas there is probably continued disease progression beyond 9 or 10 years with no surgery ${ }^{27,73)}$. There is thus strong statistical support for endorsing total resection, not only for symptomatic children but also as prophylactic treatment for asymptomatic children with lipomas, at least those with the dorsal and transitional types (see below for chaotic lipomas).

There are currently no data on the rate or probability of disease progression for adults with asymptomatic lipomas, and their actuarial life span is obviously shorter than that of children, so the cumulative risk for adults should be far smaller. A forceful argument therefore cannot be made for prophylactic surgery in asymptomatic adults, but when a lipoma becomes symptomatic, further deterioration is usually inevitable, and aggressive resection can then be justified for both children and adults.

\section{IS PARTIAL RESECTION WORSE THAN NO RE- SECTION?}

My firm answer to this question is a definitive yes. To start, the 11 years PFS probability of $34.6 \%$ from our own partial resection series of 116 lipomas ${ }^{59-61)}$ is clearly inferior to the Paris (PFS of 67\%) and London (PFS of 60\%) series ${ }^{27,73)}$ of observation alone. If we now calculate outcome only on our asymptomatic virgin cases who had partial resection to match the profiles of the other two series, in essence eliminating the fastidious redo lipomas, their 11-year PFS after partial surgery is only $43 \%{ }^{60)}$, still far short of the Paris and London numbers $^{27,61,74)}$. Moreover, the Parisian series of partial resection ${ }^{27)}$ managed only a slightly higher PFS of 51\% (Fig. 46), and other similar series report equally dismal or worse PFSs, far worse than no surgery. Minus the good-risk terminal lipomas, Colak et al.'s series ${ }^{11)}$ would have a less than 50\% PFS, as would Pierre-Kahn's partial resection series a less than $40 \% \mathrm{PFS}^{63)}$. Cochrane et al.' $\mathrm{s}^{10)}$ partial resection of transitional lipomas had a 10 -year PFS of only $20 \%$, and Cornette et al. ${ }^{12)}$ conceded unaltered progression of disease after partial resection.

The explanation for the above statistics is starkly obvious if one had the unpleasant experience of exploring lipomas that had undergone partial resection, as I did more than 150 times, and witnessed how the grotesquely contorted, heavily fat-endowed, and unneurulated placode could be solidly welded to the dura by rigid scar far worse than in an unoperated lipoma with its nascent surface. If the frequency and speed of symptomatic recurrence are proportional to the extent and firmness of fixity of the placode, one would certainly expect partial resection to incur quicker and more severe functional impairment than if no surgery had been done, which is clearly shown in our 2013 analysis $^{61)}$. The time course of in situ scar

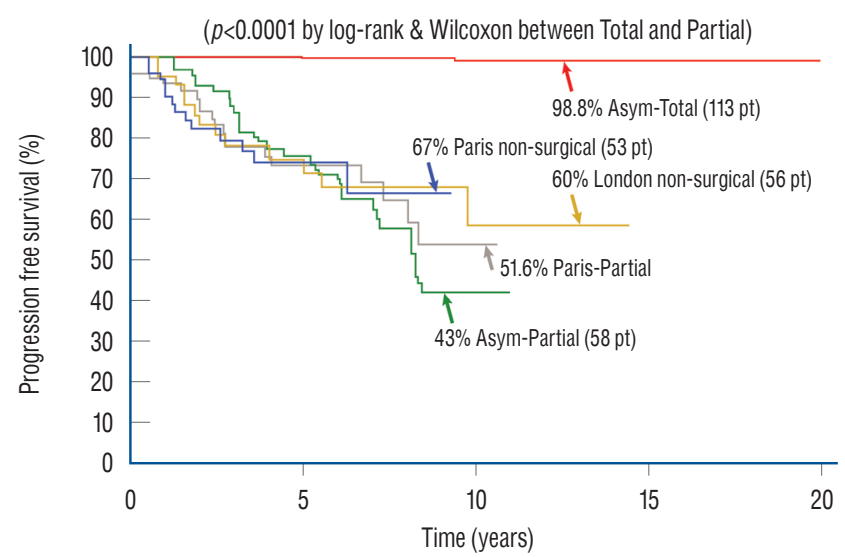

Fig. 46. Outcome differences between total resection, partial resection, and non-surgical management of asymptomatic virgin lipomas by Kaplan-Meier analysis. The non-surgical progressive-free survival graphs ("Paris non-surgical asym") from the Parisian study ${ }^{66)}$ and from the London study ${ }^{68)}$ ("London non-surgical asym") are inserted for visual comparison only and not meant to imply a true "head-to-head" comparison. Progression-free probability at 20 years for the 113 asymptomatic virgin lipomas that had undergone total resection is $98.8 \%$, much better than $67 \%$ of the Parisian series or the $60 \%$ of the London series, and far superior to the $43.3 \%$ of our own partial resection series and the $51.6 \%$ of the Parisians' own partial resection series. The difference between total and partial resection for asymptomatic virgin lipomas is highly significant $(p<0.0001)$. The Paris partial resection and our own partial resection series are as labelled. Reprinted from Pang ${ }^{55}$ with permission from the Japanese Neurosurgical Society. Pt : patients, Asym-Total : asymptomatic virgin lipomas treated by total resection, Asym-Partial : asymptomatic virgin lipomas treated by partial resection, Paris non-surgical : asymptomatic virgin lipomas managed nonsurgically in Paris ${ }^{66}$, London non-surgical asym : asymptomatic virgin lipomas from London ${ }^{68)}$. 
formation also explains why partial resection typically gives vimmediate clinical improvement owing to the initial relief of tethering but is unable to sustain these short-lived benefits against the slow but inexorable reality of chronic progressive scar formation, ending in an unrelenting downhill path that is clearly worse than the natural history of the disease.

Our extensive literature search suggests that the current global practice of lipoma surgery comprises a continuum of techniques ranging from perfunctory whittling of fat without placode refurbishment, to complete resection of lipoma, elaborate reconstruction of the placode, and capacious dural grafting, as is endorsed here. In between these two extremes, there is at least one historical series that describes the halfmeasure of "subtotal fat removal" and "partial neurulation", and reports somewhat better results than non-surgical treatment ${ }^{29)}$. The thoroughness of "clean" placode reconstruction, which is estimable by MRI, thus appears to be commensurate with the clinical benefits, and that the projected outcome of non-surgical treatment lies somewhere between the two extremes of skimpy and complete fat removal. This would also imply that partial resection will in the long run prove more harmful than no surgery for the patient. In this context, I rather strongly think that partial resection should be discouraged.

\section{NEW INSIGHTS AND SOME NEW RECOMMEN- DATIONS}

Since our 2013 report $^{61)}$, the ever-enlarging data pool on total resection has induced the author to consolidate a few recommendations regarding treatment that were, at best, tentatively entertained before.

\section{LIPOMA TYPES AND OUTCOME AND THE DUBI- OUS RANK OF CHAOTIC LIPOMAS}

According to our total resection data, the type of lipoma should not affect outcome projection ${ }^{60,61)}$, but in reality it probably does. One of the limitations of biostatistics is its inherent fallacy of rigidly assigning finite variables to represent the almost infinite vicissitudes of biological forms. For example, under the same umbrella of transitional lipomas, there are lesions with relatively orderly alignment of DREZ and nerve roots, a predictable lipoma-cord interface, and manageable quantities of fat, and there are also lipomas with plume-like billows of smothering fat that defy designation. The success and complication rates for these two extremes cannot be the same, and indiscriminately blending their divergent statistical weights is accordingly misleading. Thus, the blanket statement of "no statistical difference" in outcome between lipoma types should not be taken too literally, nor should it supplant an experienced surgeon's impression, despite comforting statistics, that some sprawling, intractable transitional lipomas demand much more exacting skill and portend a graver prognosis than a tamer, friendlier dorsal lipoma.

By far the most difficult and treacherous lipomas I have ever tackled are the chaotic lesions. The demarcation between fat and neural tissue is sometimes indiscernible even within the dorsal portion of these lipomas, and the resection should be more conservative and aim only to remove just enough dorsal fat to enable a comfortable neurulation of the dorsal part. I have repeatedly emphasised restraint and avoid doing damage to the pia covering the ventral fat since its complete

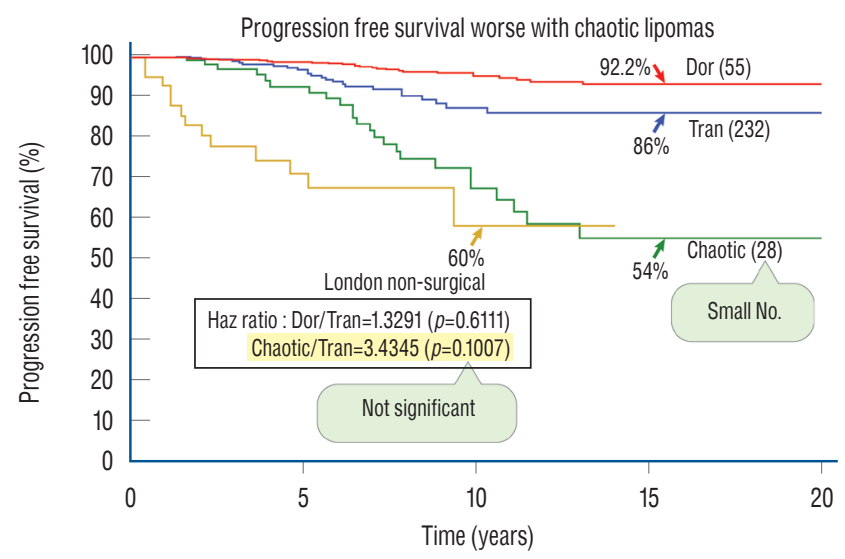

Fig. 47. The influence of lipoma type on outcome after total resection by KM analysis. The progression-free probabilities for the three lipoma types are indicated by arrows. There is no significant difference in outcome between dorsal and transitional lipomas ( $p=0.458$ by log-rank and 0.904 by Wilcoxon) even after adjusting for sample size. The hazard ratio between chaotic and transitional lipomas is not statistically significant but that may be due to the small number in the chaotic group. The London non-surgical treatment progression-free survival curve ${ }^{68)}$ is now inserted on to the 3 PFS curves of the different lipoma types obtained with the Cox proportional hazards analysis. The 10 years PFS from the London series is very similar to the PFS of the chaotic lipomas, which raises the question whether there is merit in resecting asymptomatic chaotic lipomas. Reprinted from Pang ${ }^{55}$ with permission from the Japanese Neurosurgical Society. KM : Kaplan-Meier, PFS : progression-free survival. 
resection and ventral neurulation are never possible. Consequently, it is not uncommon for chaotic lipomas to have higher cord-sac ratios and display more residual fat than the other two lipoma types on the post-operative MRI (Fig. 26D).

When in 2010 we compared the total resection outcomes of the three lipoma types in a cohort of 238 patients, we found no actual statistical differences between them ${ }^{60)}$. However, even with the caveat of small sample size of only 10 chaotic lesions, the PFS curve for chaotic lipomas ran considerably lower than the curves for dorsal and transitional lesions, and the $p$ values between chaotic and the other two lesions were only very slightly higher than 0.05 , giving us the preliminary suspicion that chaotic lipomas had worse prognosis than the others. In our 2013 series of 315 total resections ${ }^{61)}$, the number of chaotic lipomas has risen to 28 . The PFSs for dorsal and transitional lipomas of $92.2 \%$ and $86 \%$, respectively, remained very
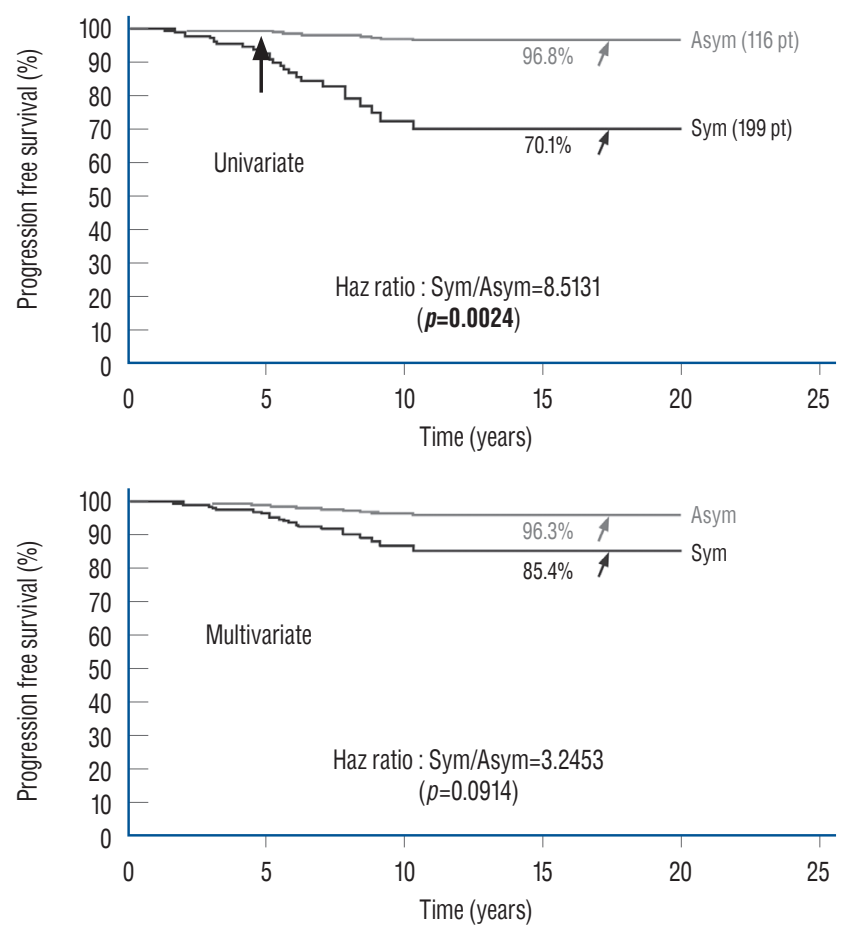

Fig. 48. Paired Cox Univariate (upper) and Multivariate (lower) analyses of the influence of pre-operative symptoms on PFS. The respective progression-free probabilities for asymptomatic and symptomatic lipomas are indicated by arrows, and their hazard ratios and relevant $p$ values are listed for each Cox analysis. This shows the individual influence (expressed in univariate analysis) of pre-operative symptoms on outcome disappears when the influences of the other predictor variables are jointly considered (by multivariate analysis). Significant $p$ values are in bold. Reprinted from Pang et al. ${ }^{61)}$ with permission from Springer Nature. Pt : patients, Asym : asymptomatic lipomas, Sym : symptomatic lipomas. similar to the 2010 figures, again without statistical difference with a $p$-value of 0.458 . However, the PFS for chaotic lesions has dropped to $54 \%$ and its regression curve separates even further away from the other two curves than in 2010 (Fig. 47). Within this chaotic group, there were no patients with cordsac ratios lower than $30 \%$, and their post-operative MRI often showed a hefty placode with a loathsome amount of remainder ventral fat and a tight container $\mathrm{fit}^{61}$. Mathematically, the outcome differences between chaotic and the other 2 lipoma types only barely reach statistical significance ( $p$-value of 0.0492 with dorsal lipoma and 0.0481 with transitional lipoma) but given larger numbers in the future, the rank of chaotic lesions might very well be definitively downgraded to the most sinister of the three lipoma types.

In Fig. 47, I inserted the London outcome curve of non-surgical treatment on to the PFSs of the 3 lipoma types. The $54 \%$ PFS of chaotic lipomas, after aggressive resection, is actually worse than both the London and Paris series of non-surgical treatment, which begs the question whether asymptomatic chaotic lipomas should be treated with any prophylactic surgery, regardless of technique. This sobering observation has changed my 2010 stance of prophylactically resecting all types of asymptomatic lipomas, and I now recommend that asymptomatic chaotic lesions, if unmistakably diagnosed on MRI, should be closely observed without surgery until symptoms develop.

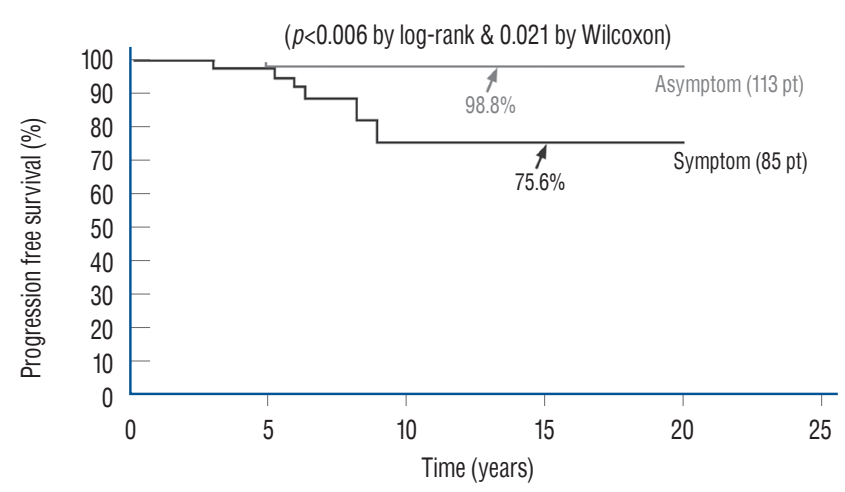

Fig. 49. The influence of pre-operative symptoms on outcome for virgin lipomas after total resection. Kaplan-Meier analysis for asymptomatic virgin and symptomatic virgin lipomas after total resection. Progressionfree probability at 20 years for asymptomatic virgin lipomas is $98.8 \%$, versus $75.6 \%$ for symptomatic virgin lipomas. The difference is significant ( $p=0.006$ by log-rank and 0.021 by Wilcoxon). Reprinted from Pang et al. ${ }^{611}$ with permission from Springer Nature. Pt : patients, Asymptom : asymptomatic virgin lipomas, Symptom : symptomatic virgin lipomas. 


\section{ASYMPTOMATIC VERSUS SYMPTOMATIC LI- POMAS}

For all the lofty statistics and extravagant analyses, there remains the unanswered question whether symptomatic lipomas do worse than asymptomatic ones after total resection. In our 2013 series of 315 patients ${ }^{611}$, multivariate analysis finds no statistical difference in outcome between symptomatic and asymptomatic lesions, but univariate analysis, in contrast, shows that asymptomatic lesions had significantly better outcome than symptomatic lesions, as we have found in our earlier series of 238 patients (Fig. 48). Even when the analysis is run just on the virgin lesions to eliminate the "redo factor" among symptomatic patients, asymptomatic patients still came out much better $^{60)}$ (Fig. 49).

Besides our own results, several large series in the literature $^{16,29,63)}$ also report considerably better outcome for asymptomatic than symptomatic patients. It was once argued that children with symptoms tend to be older and are thus more likely to undergo growth spurts and take up rigorous sports, both situations subject the spinal cord to pernicious tugging known to trigger symptoms in any tethered cord conditions, whereas asymptomatic children are much younger. The postresection profiles of the two groups are thus not comparable as the symptomatic older child is being followed at a more vulnerable age $e^{29)}$. Whilst this may explain some cases of early recurrence in the symptomatic group, Byrne et al.'s series of 100 infants, which eliminated the age difference in the two groups, still shows a markedly better outcome for asymptomatic infants ${ }^{6}$. Current consensus seems to support the notion that there are indeed prognostic differences between symptomatic and asymptomatic lipomas.

To date, no consistent dissimilarity in size, architecture, or histology has been found between symptomatic and asymptomatic lipomas, but there may be invisible differences in susceptibility inherent within their respective spinal cords. Perhaps the already injured cord that is showing symptoms has become more "fragile" and possesses a lower threshold for new symptoms with even "slight" retethering. Perhaps those spinal cords which develop early symptoms are also intrinsically predisposed to sustain new injuries to even minor future insults and are thus destined to have earlier disease recurrence.

\section{CONCLUSION}

Total/near-total resection of complex spinal cord lipomas and complete reconstruction of the neural placode produce a much better long term symptom-free survival than partial resection. Partial resection causes new and exuberant adhesions between the cut surface of the remainder fat and dura, resulting in earlier and firmer retethering and consequently a worse prognosis compared to no surgery. Our post-operative neurourological and wound complications for total resection are either comparable to, or lower than, most series of partial resection.

Total resection also confers much greater long term benefits than no surgery in children with asymptomatic virgin lipomas. The ideal patient profile for total resection predictive of early disease stabilisation and the longest recurrence-free survival is that of a child less than 2 years old who is without symptoms or prior surgery. We therefore strongly recommend prophylactic total resection for asymptomatic dorsal and transitional lipomas in children. Multivariate analysis also shows that a low post-operative cord-sac ratio is highly predictive of good long-term outcome. My firm impression is that a wellexecuted neurulation is just as important in preventing retethering.

Our experience so far shows that chaotic lipomas are the most treacherous, and the majority are simply not amenable to thorough resection or optimal placode reconstruction, let alone attaining the goal of a low cord-sac ratio. Their poor statistics are unlikely to improve even with larger numbers, and consequently I do not recommend prophylactic surgery for asymptomatic chaotic lipomas, a rather drastic change from our 2013 endorsement.

A lengthy analysis like this one is futile unless it can provide at least a few serviceable "truths". Contrary to the skewed opinion of those who had not actually seen the operation, the technical aspectsof total resection can be learned by any willing neurosurgeon with a modicum of patience, tenacity, and open-mindedness. Those who have observed the surgery will attest that other than a few unaccustomed manoeuvres and tricks, which can be learned, the rest is strictly standard microsurgery. The intraoperative electrophysiology part can be managed by any trained neurophysiologist, and the equipment should be purchasable at reasonable cost.

Last but by no means least, our massive outcome data col- 
lected over 25 years powerfully endorse total resection for asymptomatic dorsal and transitional lipomas in children and for all symptomatic lipomas in all ages. The timing of surgery for children should be soon after diagnosis, except in very young infants with stable neurology, for whom surgery is delayed till 6 to 12 months of age to minimise surgical and anaesthetic morbidity. Surgery for asymptomatic chaotic lipomas is withheld until symptoms develop.

\section{CONFLICTS OF INTEREST}

No potential conflict of interest relevant to this article was reported.

\section{INFORMED CONSENT}

This type of study does not require informed consent.

\section{AUTHOR CONTRIBUTIONS}

\author{
Conceptualization : DP \\ Data curation : DP \\ Formal analysis : DP \\ Methodology : DP \\ Project administration : DP \\ Visualization : DP \\ Writing - original draft : DP \\ Writing - review \& editing : DP
}

\section{ORCID}

Dachling Pang https://orcid.org/0000-0002-6603-6546

\section{References}

1. Arai H, Sato K, Wachi A : Surgical management in 81 patients with congenital intraspinal lipoma. Child Nerv Syst 8 : 171, 1992

2. Atala A, Bauer SB, Dyro FM, Shefner J, Shillito J, Sathi S, et al. : Bladder functional changes resulting from lipomyelomeningocele repair. J Urol 148(2 Pt 2) : 592-594, 1992
3. Barson AJ : The vertebral level of termination of the spinal cord during normal and abnormal development. J Anat 106(Pt 3) : 489-497, 1970

4. Bruce DA, Schut $L:$ Spinal lipomas in infancy and childhood. Childs Brain 5 : 192-203, 1979

5. Brunelle F, Sebag G, Baraton J, Carteret M, Martinat P, Pierre-Kahn A : Lumbar spinal cord motion measurement with phase-contrast MR imaging in normal children and in children with spinal lipomas. Pediatr Radiol 26 : 265-270, 1996

6. Byrne RW, Hayes EA, Georg TM, McLone DG : Operative resection of 100 spinal lipomas in infants less than 1 year of age. Pediatr Neurosurg 23 : 182-186; discussion 186-187, 1995

7. Caldarelli M, McLone DG, Colins JA, Suwa J, Knepper PA : Vitamin A induced neural tube defects in a mouse. Concepts Pediatr Neurosurg $6: 161-171,1985$

8. Chapman PH : Congenital intraspinal lipomas: anatomic considerations and surgical treatment. Childs Brain 9 : 37-47, 1982

9. Chapman PH, Davis KR : Surgical treatment of spinal lipomas in childhood. Pediatr Neurosurg 19 : 267-275; discussion 274, 1993

10. Cochrane DD, Finley C, Kestle J, Steinbok P: The patterns of late deterioration in patients with transitional lipomyelomeningocele. Eur J Pediatr Surg 10 Suppl 1 : 13-17, 2000

11. Colak A, Pollack IF, Albright AL : Recurrent tethering: a common longterm problem after lipomyelomeningocele repair. Pediatr Neurosurg 29 : 184-190, 1998

12. Cornette L, Verpoorten C, Lagae L, Plets C, Van Calenbergh F, Casaer $P$ : Closed spinal dysraphism: a review on diagnosis and treatment in infancy. Eur J Paediatr Neurol 2 : 179-185, 1998

13. Detwiler SR, Hotzer $H$ : The inductive and formative influence of the spinal cord upon the vertebral column. Bull Hosp Joint Dis 15 : 114-123, 1954

14. Dias $M$, Pang $D$ : Human neural embryogenesis: a description of neural morphogenesis and a review of embryonic mechanisms in Pang D (ed) : Disorders of the Pediatric Spine. New York : Raven Press, 1994

15. Dick EA, de Bruhn $R$ : Ultrasound of the spinal cord in children: its role. Eur Radiol 13 : 552-562, 2003

16. Dorward NL, Scatliff JH, Hayward RD : Congenital lumbosacral lipomas: pitfalls in analysing the results of prophylactic surgery. Childs Nerv Syst $18: 326-332,2002$

17. Hamilton HL, Boyd JD, Mossman HM : Human Embryology, ed 4. Baltimore: Williams \& Wilkins, 1972

18. Hoffman HJ, Hendrick EB, Humphreys RP : The tethered spinal cord: its protean manifestations, diagnosis and surgical correction. Childs Brain 2 : 145-155, 1976

19. Hoffman HJ, Taecholarn C, Hendrick EB, Humphreys RP : Management of lipomyelomeningoceles. Experience at the Hospital for Sick Children, Toronto. J Neurosurg $62: 1-8,1985$

20. James CC, Lassman LP : Diastematomyelia and the tight filum terminale. J Neurol Sci 10 : 193-196, 1970

21. James HE, Canty TG : Human tails and associated spinal anomalies. Clin Pediatr (Phila) 34 : 286-288, 1995

22. James HE, Williams J, Brock W, Kaplan GW, U HS : Radical removal of 
lipomas of the conus and cauda equina with laser microneurosurgery. Neurosurgery $13: 340-345,1984$

23. Jones PH, Love JG : Tight filum terminale. AMA Arch Surg 73 : 556566, 1956

24. Källén $B$ : Early embryogenesis of the central nervous system with special reference to closure defects. Dev Med Child Neurol 10(Suppl 6) : 4453, 1968

25. Kanev PM, Lemire RJ, Loeser JD, Berger MS : Management and longterm follow-up review of children with lipomyelomeningocele, 19521987. J Neurosurg $74: 48-52,1990$

26. Koyanagi I, Iwasaki Y, Hida K, Abe H, Isu T, Akino M : Surgical treatment supposed natural history of the tethered cord with occult spinal dysraphism. Childs Nerv Syst $13: 268-274,1997$

27. Kulkarni AV, Pierre-Kahn A, Zerah $M$ : Conservative Management of asymptomatic spinal lipomas of the conus. Neurosurgery 54 : 868-873; discussion 873-875, 2004

28. Kunitomo $\mathrm{K}$ : The development and reduction of the tail and of the caudal end of the spinal cord. Carnegie Contr Embryol 8 : 161-203, 1918

29. La Marca F, Grant JA, Tomita T, McLone DG : Spinal lipomas in children: Outcome of 270 procedures. Pediatr Neurosurg $26: 8-16,1997$

30. Marin-Padilla M : Clinical and experimental rachischisis in Vinken PS, Bruyn GW (eds) : Handbook of clinical neurology. Amsterdam : North-Holland, 1978, Vol 32, pp159-191

31. Marin-Padilla $\mathrm{M}$ : Mesodermal alterations induced by hypervitaminosis $\mathrm{A}$. J Embryol Exp Morphol 15 : 261-269, 1966

32. Marin-Padilla $\mathrm{M}$ : Morphogenesis of anencephaly and related malformations in Current topics in pathology. Heidelberg: Springer, 1921, pp145-174

33. Marin-Padilla M : Morphogenesis of experimental encephalocele (Cranioschisis occulta). J Neurol Sci 46 : 83-99, 1980

34. Marin-Padilla $\mathrm{M}$ : Notochordal-basichondrocranium relationships: abnormalities in experimental axial skeletal (dysraphic) disorders. J Embryol Exp Morphol 53 : 15-38, 1979

35. Marin-Padilla $\mathrm{M}$ : The tethered cord syndrome: developmental considerations in Holtzmann RNN, Stein BM (eds) : The tethered spinal cord. New York: Thieme, 1985, pp3-13

36. Marin-Padilla M, Marin-Padilla TM : Morphogenesis of experimentally induced Arnold--Chiari malformation. J Neurol Sci 50 : 29-55, 1981

37. McGuire EJ, Woodside JR, Borden TA, Weiss RM : Prognostic value of urodynamic testing in myelodysplastic patients. J Urol 126 : 205-209, 1981

38. McGuire EJ : The innervation and function of the lower urinary tract. J Neurosurg 65 : 278-285, 1986

39. McLone DG, Knepper PA : Role of complex carbohydrates and neurulation. Pediatr Neurosci 1 : 2-9, 1985-1986

40. McLone DG, Mutluer S, Naidich TP : Lipomeningoceles of the conus medullaris in Karger $S$ (ed) : Concepts in Pediatric Neurosurgery. Basel : Karger, 1982, pp171-177

41. McLone DG, Naidich TP : Laser resection of fifty spinal lipomas. Neurosurgery $18: 611-615,1986$
42. McLone DG, Naidich TP : Spinal dysraphism: Experimental and clinical in Holtzman RN, Stein BM (eds) : The Tethered Spinal Cord. New York: Thieme-Stratton, 1985

43. McLone DG, Suwa J, Collins JA, Poznaski S, Knepper PA : Neurulation: biochemical and morphological studies on primary and secondary neural tube defects. Concepts Pediatr Neurosurg 4 : 15-29, 1983

44. Morriss-Kay GM, Crutch B : Culture of rat embryos with beta-D-xyloside: evidence of a role for proteoglycans in neurulation. J Anat 134(Pt 3) : 491-506, 1982

45. Müller F, O'Rahilly R : The development of the human brain, the closure of the caudal neuropore, and the beginning of secondary neurulation at Stage 12. Anat Embryol (Berl) 176 : 413-430, 1987

46. O'Rahilly R, Meyer DB : The timing and sequence of events in the development of the human vertebral column during the embryonic period proper. Anat Embryol (Berl) 157 : 167-176, 1979

47. O'Shea KS, Kaufman MH : Phospholipase C-induced neural tube defects in the mouse embryo. Experientia 36 : 1217-1219, 1980

48. Pang $D$ : Commentary to the article: asymptomatic lumbosacral lipomas-a natural history study, by Wykes $V$, Desai $D$, and Thompson DNP. Childs Nerv Syst 28 : 1741-1742, 2012

49. Pang $D$ : Electrophysiological monitoring for tethered cord surgery in Yamada S (ed) : Tethered cord syndrome, ed 2. New York : Thieme Medical Publishers, 2010, pp199-209

50. Pang $D$ : Intraoperative neurophysiology of the conus medullaris and cauda equina. Childs Nerv Syst 26 : 411-412, 2010

51. Pang D : Spinal Cord Lipoma in Batjer H, Loftus C (eds) : Textbook of Neurological Surgery. New Jersey : Lippincott, Williams and Wilkins, 2002

52. Pang D : Spinal Cord Lipomas in Pang D (ed) : Disorders of the Pediatric Spine. New York : Raven Press, 1995, pp175-201

53. Pang $D$ : Surgical management of complex spinal cord lipomas: how, why, and when to operate. A review. J Neurosurg Pediatr 23 : 537556, 2019

54. Pang D : Tethered Cord Syndrome in Hoffman HJ (ed) : Advances in Pediatric Neurosurgery. Philadelphia : Hanley and Belfus, Inc, 1986, pp45-79

55. Pang D : Total resection of complex spinal cord lipomas: how, why, and when to operate? Neurol Med Chir (Tokyo) 55 : 695-721, 2015

56. Pang $D$, Casey $K$ : Use of an anal sphincter pressure monitor during operations on the sacral spinal cord and nerve roots. Neurosurgery 13 : 562-568, 1983

57. Pang D, Wilberger JE Jr : Tethered cord syndrome in adults. J Neurosurg $57: 32-47,1982$

58. Pang D, Zovickian J, Moes GS : Retained medullary cord in humans: late arrest of secondary neurulation. Neurosurgery 68 : 1500-1519; discussion 1519, 2011

59. Pang D, Zovickian J, Oviedo A : Long-term outcome of total and neartotal resection of spinal cord lipomas and radical reconstruction of the neural placode: part I-surgical technique. Neurosurgery 65 : 511-528; discussion 528-529, 2009

60. Pang D, Zovickian J, Oviedo A : Long-term outcome of total and near- 
total resection of spinal cord lipomas and radical reconstruction of the neural placode, part II: outcome analysis and preoperative profiling. Neurosurgery 66 : 253-272; discussion 272-273, 2010

61. Pang D, Zovickian J, Wong ST, Hou YJ, Moes GS : Surgical treatment of complex spinal cord lipomas. Childs Nerv Syst 29 : 1485-1513, 2013

62. Pierre-Kahn A, Lacombe J, Pichon J, Giudicelli Y, Renier D, Sainte-Rose C, et al. : Intraspinal lipomas with spina bifida. Prognosis and treatment in 73 cases. J Neurosurg 65 : 756-761, 1986

63. Pierre-Kahn A, Zerah M, Renier D, Cinalli G, Sainte-Rose C, LellouchTubiana $A$, et al. : Congenital lumbosacral lipomas. Childs Nerv Syst 13 : 298-334; discussion 335, 1997

64. Sathi S, Madsen JR, Bauer S, Scott RM : Effect of surgical repair on the neurologic function in infants with lipomeningocele. Pediatr Neurosurg $19: 256-259,1993$

65. Schoenwolf GC : Histological and ultrastructural observations of tail bud formation in the chick embryo. Anat Rec 193 : 131-147, 1979

66. Schoenwolf GC : Histological and ultrastructural studies of secondary neurulation in mouse embryos. Am J Anat 169 : 361-376, 1984

67. Schut L, Bruce DA, Sutton LN : The management of the child with a lipo- myelomeningocele. Child Neurosurg $30:$ 464-476, 1983

68. Stolke $D$, Zumkeller $M$, Seifert $V$ Intraspinal lipomas in infancy and childhood causing a tethered cord syndrome. Neurosurg Rev $11:$ 59-65, 1988

69. Streeter $G L$ : Factors involved in the formation of the filum terminale. Am J Anat $25: 1-11,1919$

70. Sutton LN : Lipomyelomeningocele. Neurosurg Clin N Am 6 : 325338, 1995

71. Talwalker VC, Datsur DK : Ectopic spinal cord myelomeningocele with tethering: a clinicopathological entity. Dev Med Child Neurol 16 (Suppl 32) : 159-160, 1974

72. Toole BP : Glycosaminoglycans in morphogenesis in Hay E (ed) : Cell Biology of Extracellular Matrix. New York : Plenum Press, 1981, pp229-294

73. Wykes V, Desai D, Thompson DN : Asymptomatic lumbosacral lipomas-a natural history study. Childs Nerv Syst 28 : 1731-1739, 2012

74. Xenos C, Sgouros S, Walsh R, Hockley A : Spinal lipomas in children. Pediatr Neurosurg 32 : 295-307, 2000 